\title{
PLANNING OF A HYBRID ENERGY SYSTEM CONNECTED TO A DISTRIBUTION SYSTEM
}

\author{
A Dissertation \\ by \\ AHMAD MOHAMMAD AMIN ABU-ELRUB \\ Submitted to the Office of Graduate and Professional Studies of \\ Texas A\&M University \\ in partial fulfillment of the requirements for the degree of \\ DOCTOR OF PHILOSOPHY
}

$\begin{array}{ll}\text { Chair of Committee, } & \text { Chanan Singh } \\ \text { Committee Members, } & \text { Le Xie } \\ & \text { Laszlo Kish } \\ & \text { Sergiy Butenko } \\ \text { Head of Department, } & \text { Miroslav Begovic }\end{array}$

December 2016

Major Subject: Electrical Engineering

Copyright 2016 Ahmad Abu-Elrub 


\begin{abstract}
Global warming and excessive use of depletable energy resources are considered as some of the most serious problems that need to be solved. As a response, renewable energy sources (RES) have been gaining an increasing importance in recent years. The electrical power generated from RES tends to be fluctuating due to intermittency of wind speed and solar radiation which may result in frequency and voltage deviations in the grid as well as loss of the capacity to serve the load. Distributed generation of windphotovoltaic hybrid systems connected to energy storage (ES) is proposed to overcome the variability of RES. However, the high cost of the equipment for such systems is also a challenge.
\end{abstract}

In this dissertation, planning of a hybrid wind-photovoltaic energy system combined with ES connected to a distribution system is described. The planning procedure is done in two different configurations. In the first one, the objective is to find optimal ES size for a wind farm. The objective function measures the economic benefit gained from selling of the dispatched power to the grid against the cost of ES. The result of the optimization gives the optimal size of ES. A probability distribution function (pdf) is created to represent the optimal size of ES for the planning horizon and then a case study for one year is conducted to illustrate the proposed method. For the second configuration, optimization techniques which use linear programming, two stage stochastic programming and scenario aggregation are deployed in order to find the optimal plan for the hybrid energy system. The proposed procedure uses historical wind 
speed, solar radiation, energy market price, and load data to determine the system design. The objective function measures the total annual cost of the proposed system. The results of the optimization procedure give the optimal size for wind farm, solar farm, and ES needed to meet the load requirements, minimize the annual cost, and consider system uncertainties and system reliability. 


\section{DEDICATION}

To

My Father; Mohammad, My Mother; Samiha, My Family, and My Friends. 


\section{ACKNOWLEDGEMENTS}

All thanks and praise is due to Allah (God) the Almighty, the Most Gracious, the Most Merciful.

I would like to thank my advisor Dr. C. Singh for his help and support throughout this work. His guidance helped the completion of this work.

My sincere appreciation to my committee members Dr. L. Kish, Dr. L. Xie, and Dr. S. Butenko for their help and advice during my graduate studies at Texas A\&M University.

Finally, I would like to thank all my family members for their continuous support and tremendous help in all steps of my life. 


\section{TABLE OF CONTENTS}

Page

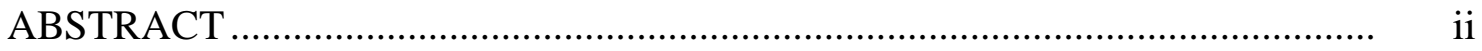

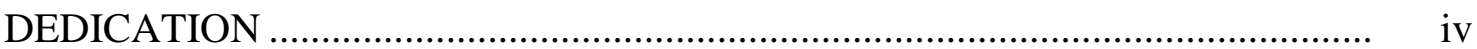

ACKNOWLEDGEMENTS ......................................................................

TABLE OF CONTENTS ............................................................................ vi

LIST OF FIGURES ............................................................................ ix

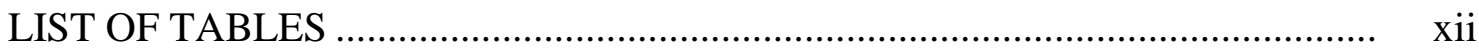

CHAPTER I INTRODUCTION.......................................................... 1

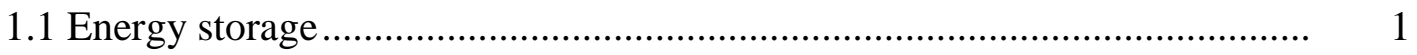

1.2 Hybrid energy systems ..................................................................... 4

CHAPTER II SIZING OF AN ENERGY STORAGE IN AN ENERGY

BUFFER SYSTEM .............................................................................

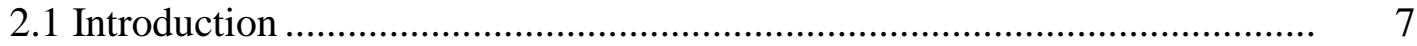

2.2 The energy buffer storage system ..................................................... 10

2.3 Determination of BESS power and energy capacities.............................. 12

2.4 Optimization to determine dispatched power......................................... 14

2.5 Optimization problem constraints ........................................................ 15

2.5.1 DC-link voltage constraint ......................................................... 15

2.5.2 State of charge ............................................................................. 19

2.6 Determination of BESS capacity ...................................................... 21

2.7 Descriptions of methods and results..................................................... 22

2.7.1 First method: probability of sufficient storage method ...................... 22

2.7.1.1 Chi-square test................................................................. 24

2.7.2 Second method: cost based method ............................................... 29

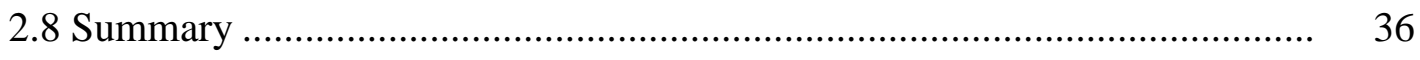

CHAPTER III PLANNING OF A HYBRID ENERGY SYSTEM .................... 37

3.1 Introduction ................................................................................... 37

3.2 Hybrid energy system description .................................................. 40 
3.2.1 Wind farm model ........................................................................ 40

3.2.2 Solar farm model ...................................................................... 42

3.2.3 Energy storage model.................................................................... 43

3.2.4 Energy market model ..................................................................... 44

3.2.5 Load model ............................................................................... 45

3.3 Problem formulation ......................................................................... 46

3.3.1 Objective function ..................................................................... 46

3.3.2 Problem constraints ....................................................................... 47

3.4 Case study ................................................................................ 52

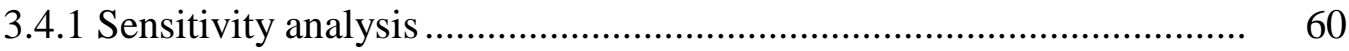

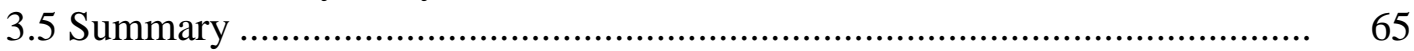

CHAPTER IV PLANNING OF A HYBRID WIND-PV DISTRIBUTION

SYSTEM CONSIDERING SYSTEM UNCERTAINTIES ................................ 67

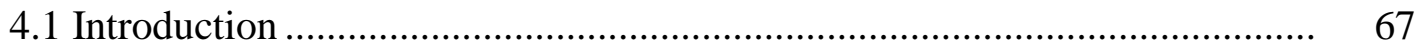

4.2 Hybrid energy system description and subsystem models ............................ 71

4.2.1 Wind farm …………..............................................................

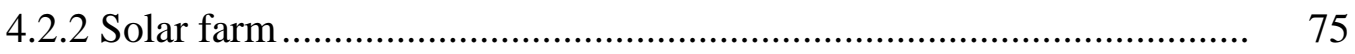

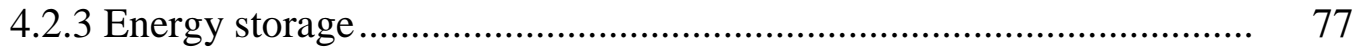

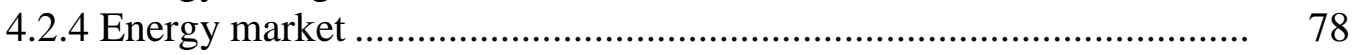

4.2.5 Load model ........................................................................... $\quad 80$

4.3 Problem formulation .......................................................................... 81

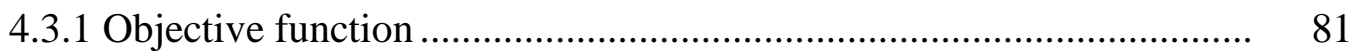

4.3.2 Problem constraints ......................................................................... 83

4.4 Solution using stochastic programming ……………................................ $\quad 88$

4.4.1 Compact matrix form …………………………………………...... 88

4.4.2 Sample average approximation .......................................................... 89

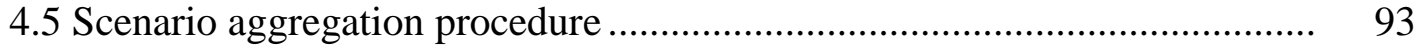

4.5.1 Cluster centers …………………………………...................... 94

4.5.2 K-means algorithm ....................................................................... 94

4.6 System reliability modeling …………………………………………..... 95

4.6.1 Expected energy not served from wind farm ..................................... 95

4.6.2 Expected energy not served from solar farm....................................... 97

4.6.3 Expected energy not served from energy market................................. $\quad 97$

4.6.4 Expected energy not served from energy storage ............................... $\quad 98$

4.7 Case study and results ............................................................................... 99

4.7.1 Case one: fully reliable system......................................................... 103

4.7.2 Case study two: considering system reliability ................................... 111

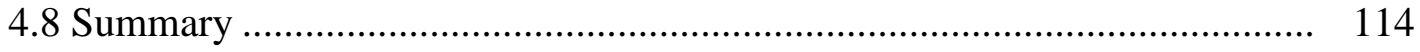


CHAPTER V CONCLUSIONS AND REMARKS ....................................... 115

REFERENCES ............................................................................... 118 


\section{LIST OF FIGURES}

Page

Figure 1.1: $\quad$ Advanced lead-acid batteries energy storage................................ 2

Figure 1.2: $\quad$ Hybrid energy systems.................................................... 4

Figure 2.1: $\quad$ Interconnection between wind turbine and the main grid............ 10

Figure 2.2: $\quad$ Third-order lead-acid battery model ......................................... 16

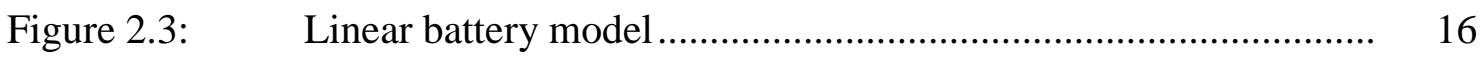

Figure 2.4: $\quad$ Frequency distribution of optimal BESS size ............................ 23

Figure 2.5: $\quad$ Frequency distribution of optimal $P_{d}$ level ............................. 23

Figure 2.6: $\quad$ System used in the second method ...................................... 30

Figure 2.7: $\quad$ Relationship between BESS size and capital cost of BESS in $\$ . . \quad 30$

Figure 2.8: $\quad$ Relationship between BESS size and fuel cost per year in \$....... 31

Figure 2.9: $\quad$ Relationship between BESS size and system cost per year in $\$ \ldots \quad 32$

Figure 2.10: $\quad$ Optimization procedure flow chart ...................................... 32

Figure 2.11: $\quad$ Wind power profile for one day ........................................... 33

Figure 2.12: $\quad$ Battery power profile ......................................................... 34

Figure 2.13: $\quad$ SOC of the battery with and without control ............................. 34

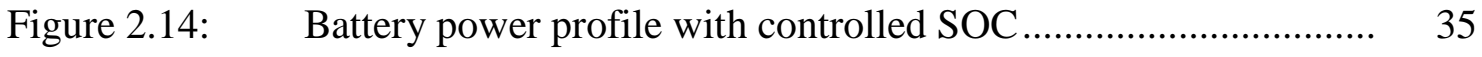

Figure 2.15: $\quad$ DC-link voltage with controlled SOC .................................. 35

Figure 3.1: $\quad$ Proposed hybrid energy system connected to distribution system 40

Figure 3.2: $\quad$ Wind farm utilization factor ............................................. 53 
Figure 3.3: $\quad$ Solar farm utilization factor ................................................. 54

Figure 3.4: $\quad$ Energy price over the time................................................... 55

Figure 3.5: $\quad$ Averaged load over the time .................................................. 55

Figure 3.6: $\quad$ Estimated range of wind LCOE projections across 18 scenarios . 57

Figure 3.7: $\quad$ Estimated solar farm capital cost projections for utility scale ...... 58

Figure 3.8: $\quad$ Power charged into energy storage ...................................... 59

Figure 3.9: $\quad$ Power discharged from energy storage .................................. 59

Figure 3.10: $\quad$ Power purchased from energy market ..................................... 60

Figure 3.11: $\quad$ Optimal wind farm size Vs. load factor .................................. 61

Figure 3.12: $\quad$ Optimal solar farm size Vs. load factor ................................... 61

Figure 3.13: $\quad$ Optimal energy storage power capacity Vs. load factor.............. 61

Figure 3.14: $\quad$ Optimal energy storage energy capacity Vs. load factor ............. 62

Figure 4.1: $\quad$ Proposed hybrid energy system connected to distribution system 71

Figure 4.2: $\quad$ Scenario aggregation flow chart ......................................... 93

Figure 4.3: $\quad$ Wind farm utilization factor .................................................. 101

Figure 4.4: $\quad$ Solar farm utilization factor ............................................ 102

Figure 4.5: $\quad$ Energy price over the time............................................... 102

Figure 4.6: $\quad$ Averaged load over the time .............................................. 103

Figure 4.7: $\quad$ Scale parameter for Weibull distribution ............................... 104

Figure 4.8: $\quad$ Shape parameter for Weibull distribution............................... 105

Figure 4.9: $\quad \alpha$ parameter for Beta distribution....................................... 105 
Figure 4.10: $\quad \beta$ parameter for Beta distribution....................................... 105

Figure 4.11: $\quad$ Log mean parameter for Lognormal distribution ..................... 106

Figure 4.12: $\quad$ Log standard deviation parameter for Lognormal distribution..... 106

Figure 4.13: $\quad$ Mean parameter for Normal distribution ............................... 106

Figure 4.14: $\quad$ Standard deviation parameter for Normal distribution ................ 107 


\section{LIST OF TABLES}

Page

Table 2.1: $\quad$ Frequency distribution for optimal BESS size ........................... 24

Table 2.2: $\quad$ The probabilities and expected frequencies using pdf model......... 26

Table 2.3: $\quad$ Normalized error squared for Chi-Square test ............................. 27

Table 3.1: $\quad$ Decision variables summary ....................................................... 51

Table 3.2: $\quad$ Wind turbine characteristics ................................................... 53

Table 3.3: $\quad$ Results for case study …...................................................... 56

Table 3.4: $\quad$ Results for scenarios two .................................................... 62

Table 3.5: $\quad$ Results for scenarios three and four....................................... 63

Table 4.1: $\quad$ Coefficients of wind turbine failure probability .......................... 96

Table 4.2: $\quad$ Wind turbine characteristics ................................................. 100

Table 4.3: $\quad$ Lower bound estimate from stochastic programming .................. 107

Table 4.4: $\quad$ Upper bound estimate from stochastic programming .................... 108

Table 4.5: $\quad$ Suboptimal planning result from scenario aggregation .................. 109

Table 4.6: $\quad$ Clusters of scenario aggregation procedure ............................... 109

Table 4.7: $\quad$ Sensitivity analysis results for stochastic programming ............... 110

Table 4.8: $\quad$ Sensitivity analysis results for scenario aggregation procedure ...... 111

Table 4.9: $\quad$ Results for case study two ................................................. 112

Table 4.10: $\quad$ Results for case study two with highly reliable energy market ....... 113 


\section{CHAPTER I}

\section{INTRODUCTION}

\subsection{Energy storage}

Global warming and excessive use of non-renewable energy resources are considered as some of the most serious problems that need to be solved. Therefore, renewable energy sources (RES) have been gaining an increasing importance in recent years. However, the power generated from renewables tends to be fluctuating due to intermittency of their resources which varies in unpredictable ways. These fluctuations may result in frequency and voltage deviations in the grid as well as inability of the system to satisfy the customer loads. In this research effort, hybrid distributed generation of wind-photovoltaic connected to energy storage is proposed to overcome the intermittency of RES.

The electric power grid in the U.S. and other countries are being transformed into a more reliable, secure, and economically efficient smart grid. Within this smart gird, large scale energy storage is included to smooth out the fluctuations of electrical power generated from the renewables. Energy storage has different types and techniques. One of these types is the battery energy storage system (BESS) which is more widely used these days. BESS has been used for smoothing electrical power output from wind and solar farms. Different large scale energy storage technologies are being studied. A 115 MW compressed air energy storage (CAES) demonstration power plant placed in service in the early 1990s has proven to be effective as shown in [1]. CAES systems could to be 
practical in a power range from above $100 \mathrm{MW}$ up to several thousand MW. The Vanadium redox-flow batteries (VRBs) are used as energy storage in addition to a wash out filter based scheme in order to smooth out the fluctuation of the power generated from wind farms [2]. Control and sizing of Zinc bromine flow batteries for wind farms have been proposed and illustrated using optimal control for a generic battery in [3].

Lead acid batteries have been deployed as energy storage. Lead acid batteries are one of the oldest and most commercially mature form of rechargeable battery technology. Lead acid battery technologies are being used in wide range of applications like automotive, uninterruptible power supplies, and marine. In Figure 1.1, 1280 advanced lead-acid batteries are used as an energy storage which can store up to 250 KW or 1 MWh of energy [4]. In Tappi Wind Park installed in 2001 by Hitachi, advanced lead acid batteries have been integrated with wind generating farms [5]. Energy storage in sizes of 10 to $20 \mathrm{MW}$ has been achieved using lead acid carbon technology [6].

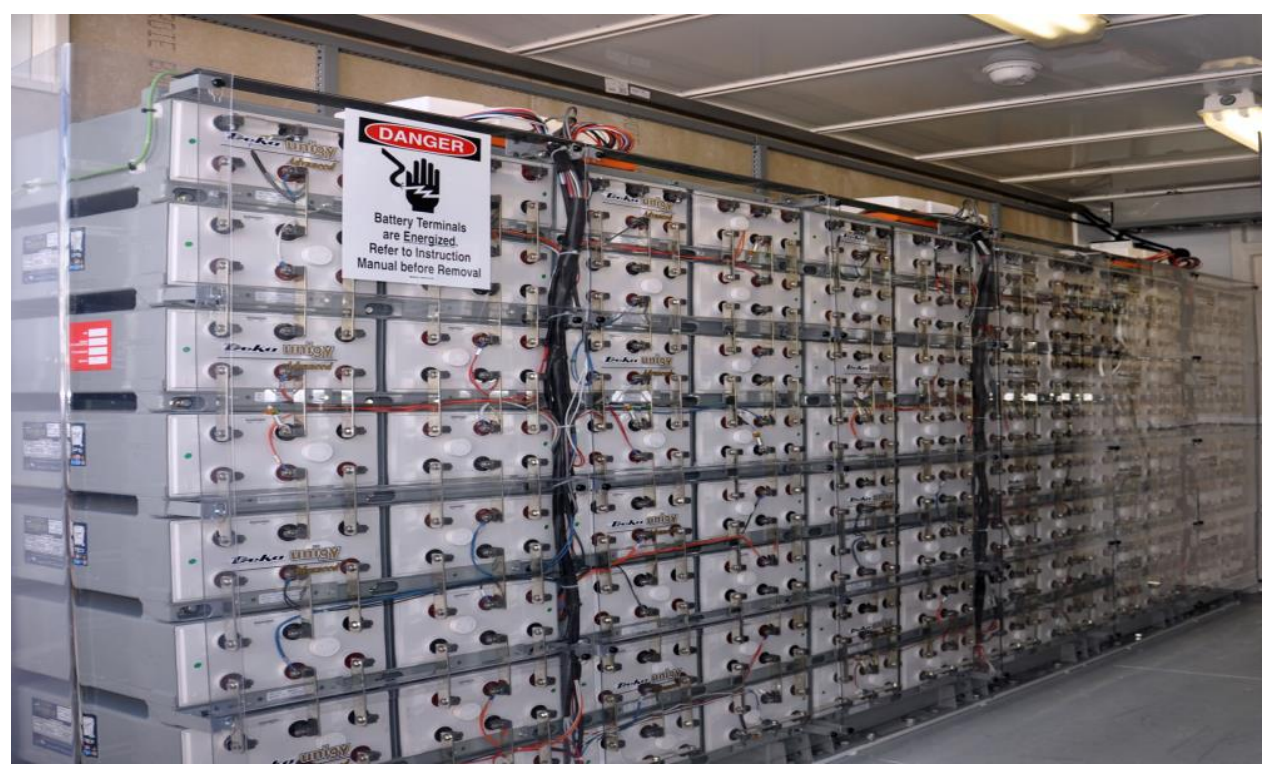

Figure 1.1 Advanced lead-acid batteries energy storage [4]. 
The BESS is shown to be a suitable technique for the long term load tracking operation because of its higher energy capacity compared to other energy storage technologies. Moreover, BESS is found also to be cost effective for use in power systems. The optimal size of BESS can be found to be varying due the varying nature of power generated from renewables. Thus, the planning of energy storage system connected to renewable resources, to smooth its output and dispatch power on hourly basis, needs a careful analysis. Hence, a criteria needs to be used to determine the proper size that is applicable over the planning horizon.

In chapter II, a novel procedure is proposed to find the optimal size of lead acid BESS connected to a wind farm. Two methods which are the probability of sufficient storage method and cost based method, have been developed to find the optimal planning of energy storage. In the first one, the optimal size of BESS connected to a wind farm, which maximize the benefit from BESS utilization, is found for each day in one year. A probability distribution function is then created from the optimal BESS size of 365 days. Then, the optimal size is chosen such that it meets the system requirements with certain specified probability. In the second one, the overall system cost is mathematically formulated as a function of BESS size. Then, optimal BESS size is defined as the BESS size that minimizes the overall system cost. 


\subsection{Hybrid energy systems}

Air pollution due to greenhouse gases released from fossil fuel generators has been increasing. Therefore, new approaches towards generating electricity with less use of fossil fuel are needed. Hence, renewable resources of energy, such as wind, solar, and hydropower have gained much attention as alternatives for electrical power generation in recent years. Hybrid energy systems, Figure 1.2, consist of one or more complementary renewable resources combined with conventional sources and some types of energy storage. The idea of the hybrid energy systems is to combine diversified energy sources (renewable and conventional) to deliver reliable electric power to the load.

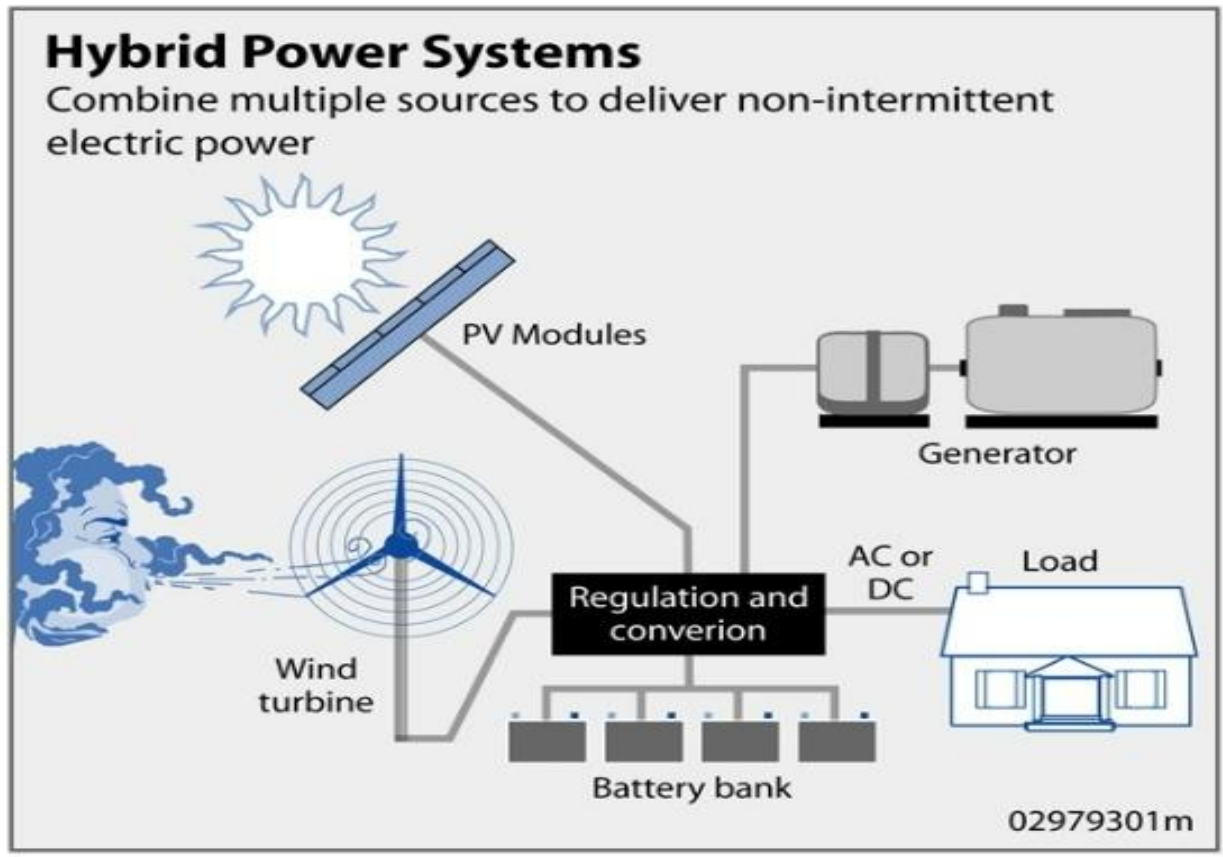

Figure 1.2: Hybrid energy systems [7]. 
The integration of renewable energy resources (RES) into the current distribution systems is considered a challenging task. The cost of equipment and the variability of the generated electrical power make RES integration difficult. Energy storage is proposed to overcome the intermittency of RES by smoothing RES output power. Yet, the high cost of RES equipment for such systems is also a disadvantage of hybrid energy systems. Therefore, a planning procedure which considers capital costs of the system components and the uncertainties of RES is needed. Furthermore, system reliability is also a vital point in planning such a system.

Considerable work has been done to find the optimal planning of hybrid energy systems. Optimization and planning of the hybrid energy system aim to obtain reasonable use of the renewable resources in order to satisfy lowest investment, load requirements, system uncertainties and system reliability. In [8], the authors develop a procedure to find optimal planning for hybrid energy system which reduces overall system cost using Biogeography Based Optimization (BBO). A stochastic framework, using a pattern search-based optimization combined with a sequential Monte Carlo simulation, is developed to find optimal planning of hybrid power system which minimize the system cost and satisfy the reliability requirements [9].

However, in the previous mentioned and other studies, the importance of time domain is not considered in the planning of the hybrid energy system. While considering renewable resources, load requirements and energy price are highly related with time. Wind and solar power typically complement each other in the time domain. Therefore, in chapter III, an optimization technique using linear programming which includes one year 
worth's of wind speed, solar radiation, energy market price, and load data in time domain is proposed to find the optimal configuration of hybrid energy systems. The optimal planning minimizes the overall system cost and meets system constraints. The overall system cost includes capital cost of system components, operation and maintenance cost, and energy purchased from the market cost.

In chapter IV, the uncertainties and the reliability of the system suggested in chapter III are considered. Two optimization techniques are used to find optimal planning of hybrid energy system. One uses two stage stochastic programming in order to find the optimal planning strategy for the hybrid system. The other is based on scenario aggregation. The stochastic programming procedure uses time correlated stochastic distributions for system uncertainties which are wind speed, solar radiation, energy market price, and load. The objective function measures the total annual cost of the system. The results of the stochastic optimization procedure give the optimal size for wind farm, solar farm, and energy storage needed to meet the load requirements, improve system reliability, and minimize system cost. The scenario aggregation procedure finds the optimal configuration of the proposed system for each scenario. Then, the optimal configuration is defined as the average of these scenarios over a sufficient number of simulated scenarios. The results from the two procedures are compared and some conclusions are pointed out. 


\section{CHAPTER II}

\section{SIZING OF AN ENERGY STORAGE IN AN ENERGY BUFFER SYSTEM*}

\subsection{Introduction}

The dispatching of electrical power, as constant as possible, generated from wind farms is important so that wind farm acts like other typical generation resources. The electrical power produced from wind farms fluctuates and this variable nature can cause voltage and frequency deviations in the grid [10]. Therefore, connecting fluctuating power source to a grid can produce challenges [11]. One of the effective solutions proposed for the problem of intermittency of electrical power generated from wind farms is energy storage.

Energy storage has different types and techniques. One of these types is the battery energy storage system (BESS) which is now more widely used. In the published literature, BESS has been used for smoothing electrical power output from wind farms. The Vanadium redox-flow batteries (VRBs) are used as energy storage in addition to a wash out filter based scheme in order to smooth out the fluctuation of the power generated from wind farms [2]. In [12-14], a simple scheme to charge and discharge the BESS by storing extra energy if the wind power goes beyond predetermined threshold is proposed.

*@ 2014 IEEE. Reprinted, with permission, from A. Abuelrub and C. Singh, "Long Term Energy Storage Capacity Optimization in Energy Buffer System," IEEE PES General Meeting | Conference \& Exposition, July, 2014. (C) 2016 IEEE. Reprinted, with permission, from A. Abuelrub and C. Singh, "Sizing of Lead Acid Storage System in an Energy Buffer Connected to a Wind Farm," IEEE International Conference on Power Systems Technology (Powercon), September, 2016. 
Research is being done to find the optimal size of BESS connected with a wind farm in order to make its power output as constant as possible. In $[15,16]$, the use of BESS with wind farm and stand-alone diesel systems is discussed. The utilization of BESS with a single wind turbine to reduce the harmful consequences of wind power changes is also examined in $[17,18]$. Control and sizing of Zinc bromine flow batteries for large wind farms have been proposed and illustrated using optimal control for a generic battery in $[3,19]$. In [20], a BESS design and modeling for 50-MW and 80-MW wind farms is discussed.

The objective in this work is to find the optimal size of BESS, from economic point of view, in order to address its design in an energy buffer system consisting of the BESS and a wind farm. In [21], the BESS is shown to be a suitable technique for the long term load tracking operation because of its higher energy capacity compared to other energy storage media. Moreover, the BESS is found to be cost effective for use in power systems as illustrated in [22]. A design method for a flow battery system is proposed in [23], however the authors did not provide a procedure to find the required capacity for the proposed energy storage system. This work proposes a procedure to find the optimal value for BESS capacity such that the BESS can meet the daily load tracking requirements and make the maximum economic benefit from the BESS. The calculated size of BESS would be capable of meeting the demands of every day in the planning horizon. The proposed procedure in this work uses the electrical power profile, generated from wind speed data from a wind farm for one year. Then using the optimal value of an optimization procedure the optimal value for the steady dispatched power 
from the wind farm for each day is obtained. The BESS capacity then will be calculated from the dispatched power level for each day, provided that the dc voltage across the DC-link capacitor and the state of charge (SOC) of the batteries will be kept between predefined limits for proper energy buffer operation. A frequency distribution of the optimal BESS size for all the days in one year will be created and converted to a probability distribution function (pdf). Finally, the optimal size of BESS will be calculated from another optimization problem which minimizes the costs related to the BESS size as shown in the next sections. The optimal size obtained is expected to be valid over the entire planning horizon. 


\subsection{The energy buffer storage system}

In the simple energy buffer system shown in Figure 2.1, taken from [24], the function of wind turbine is to generate electrical power $\left(P_{w}\right)$ from wind by converting the kinetic energy of the wind to electrical energy using permanent magnet synchronous generator (PMSG). The wind power is known to be intermittent and stochastic since it depends on wind speed which varies stochastically. Therefore, this wind power can be modeled using its statistical mean [25]. In our work, the main goal is to propose a procedure to find the optimal size of BESS that is applicable over the entire planning horizon and not just one day. Hence wind speed data for one year of wind farm is obtained and this data will be used in the procedure to determine the optimal size of the BESS in the energy buffer system.

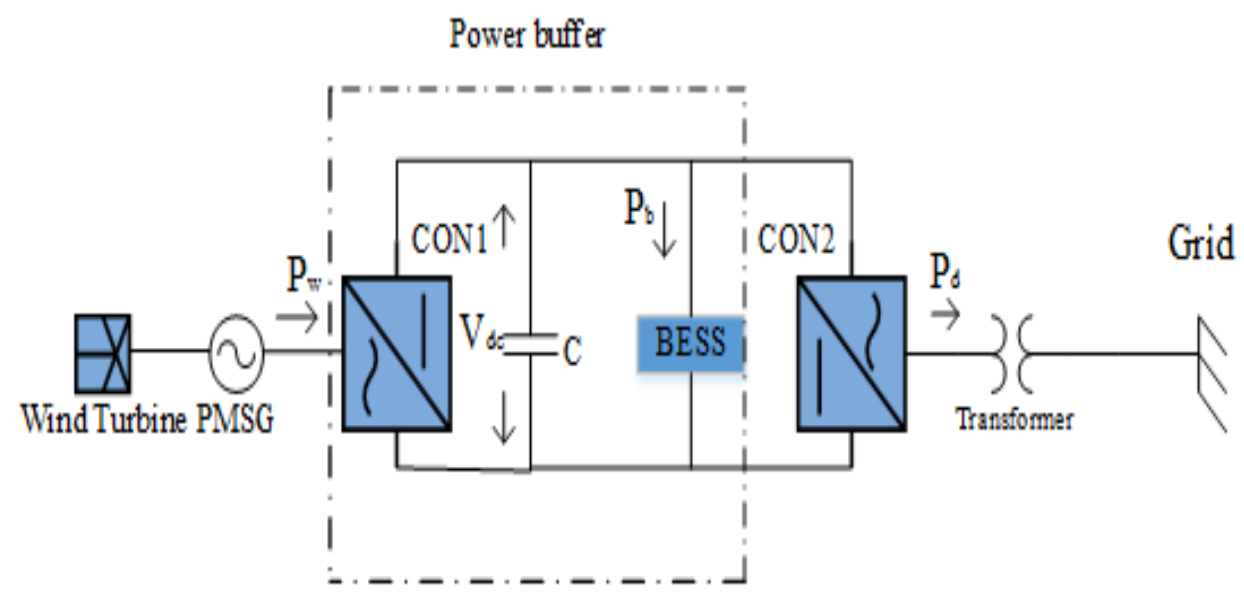

Figure 2.1 Interconnection between wind turbine and the main grid [24]. 
Back-to-back converters along with a dc link capacitor $C$ are used to transfer electrical power from wind turbine output to the grid transformer. The purpose of dc link capacitor is to filter out the harmonics of the electrical power and it can also act as an energy storage device as shown in [26]. However, the energy stored in the capacitor is very small compared to the energy stored in the BESS. Therefore, it can be neglected in the calculations. The electrical power lost in the converters is very small compared with the generated power, thus it can be also neglected. The wind power dispatched to the grid will be kept constant, as much as possible, during the day. The dispatched wind power for a given day will be constant and derived using an economic optimization procedure that uses the wind profile for that day. So the dispatched power from the wind farm is related to the wind power profile and not the required load as the dispatched power is assumed to be much smaller than the required load. In the case of power generated from wind farm is more than the required dispatched power, the extra power will be stored in the BESS. On the other hand, when the power generated from wind farm is less than the required dispatched power, the shortage in $P_{d}$ will be compensated by the energy stored in the BESS. Hence, the quality of the power generated from wind farm and dispatched to the electrical grid will be improved by reducing its fluctuation. The power convertor CON1 and the BESS together form the power buffer system which is suggested to reduce the fluctuation in wind power and make the dispatched power as smooth as possible. The operating and control procedures of the power convertors are illustrated more in details in [27]. 


\subsection{Determination of BESS power and energy capacities}

The capital cost of the lead acid battery energy storage system is found to be a function of both BESS power capacity and BESS energy capacity [28]. Therefore, the power and energy capacities of the BESS need to be determined in order to calculate BESS cost. The definition of the power capacity of the battery is the measure of battery capability to supply or store electrical power under charging and discharging limitations. The energy capacity of the battery is defined as the amount of energy that can be stored or supplied from the battery. In this section, the energy capacity of BESS will be assumed 8 times its power capacity which is suitable for wind power integration application (i.e. if BESS size is found to be $1 \mathrm{MW}$ then its energy capacity is $8 \mathrm{MWH}$ ). After defining the power and energy capacities of the battery, these capacities will be used in calculating the capital cost of the BESS. The capital cost of BESS then will be used in the optimization problem to find the optimal size of the BESS based on costbenefit analysis, as shown later.

In the energy buffer system shown in Figure 2.1, the BESS power $P_{b}(t)$ can be calculated easily, neglecting the power losses in the power convertors as illustrated earlier, as the difference between the power generated from wind farm and the power level dispatched to the grid as in Equation (2.1),

$$
P_{b}(t)=P_{w}-P_{d}
$$


Now, assuming the dispatched power $P_{d}$ to be constant, the BESS power profile will be the same as the wind power profile but shifted by constant value $P_{d}$. The power capacity of the BESS $\left(P_{b, \max }\right)$ can be calculated from the BESS power $P_{b}(t)$ and it is equal to the maximum of the absolute value of $P_{b}(t)$ as in Equation (2.2),

$$
P_{b, \text { max }}=\max \left|P_{b}(t)\right|
$$

The energy profile of the BESS $E_{b}(t)$ can be found by integrating its power profile $P_{b}(t)$ over the period of time as shown in Equation (2.3),

$$
E_{b}(t)=\int P_{b}(t) d t
$$

And then the energy capacity of BESS $\left(E_{b, \max }\right)$ can be found from Equation (2.3) since it is equal to the maximum of the absolute value of $E_{b}(t)$ and it is shown in Equation (2.4),

$$
E_{b, \max }=\max \left|E_{b}(t)\right|
$$

From the previous analysis and equations, one can note that for different values of dispatched power to the grid $P_{d}$, there are corresponding power and energy capacities of BESS $P_{b, \max }$ and $E_{b, \max }$ which will be used in the optimization problem as shown later. 


\subsection{Optimization to determine dispatched power}

The optimization formulation has an objective function which measures the economic benefit from selling the electrical power generated from wind farm to the grid against the amortized BESS capital cost per hour for period T. The optimal solution of this optimization problem gives the level of dispatched power to the grid in order to maximize the objective function, taken from [24] and shown in Equation (2.5),

$$
B=\alpha P_{d}-\beta P_{b, \max }-\gamma E_{b, \max }
$$

Where $B$ is the benefit from energy buffer system in $\$, \alpha$ is the unit price of the wind power sold to the grid (in $\$ / \mathrm{KW}$ ), $\beta$ (in $\$ / \mathrm{KW}$ ) and $\gamma$ (in $\$ / \mathrm{KWh}$ ) are the amortized BESS capital costs for BESS power and energy capacities. From the objective function represented by Equation (2.5), it can be shown that the value of the objective function depends only on the level of dispatched power $P_{d}$, since it has been proofed in the previous discussion that $P_{b, \max }$ and $E_{b, \max }$ are related to the value of $P_{d}$ (i.e. for each value of $P_{d}$ there is a corresponding values for $P_{b, \max }$ and $E_{b, \max }$ respectively). Hence, the solution of this optimization problem, for given values of $\alpha, \beta$, and $\gamma$, gives the optimal value of $P_{d}\left(P_{d}{ }^{*}\right)$ that maximizes the objective function $B$. Thus, the optimal size of the BESS that maximizes the economic benefit from BESS for a given wind profile can be calculated using this optimal value of $P_{d}$. 


\subsection{Optimization problem constraints}

\subsubsection{DC-link voltage constraint}

The DC-link voltage $\left(V_{d c}\right)$ across the DC-link capacitor must be kept between certain limits so that the power convertor can operate properly. Therefore, a verification of BESS capacity is required in order to determine if this capacity is sufficient to maintain the $V_{d c}$ between these limits as wind power changes. A mathematical method to calculate $V_{d c}$, using circuit analysis of lead acid battery model, will be used as illustrated in the following discussion.

\section{A) Battery model}

In Figure 2.2, a third order lead acid battery model, taken from [29], is shown. However, the circuit components of this model are nonlinear, therefore the following assumptions are used to simplify the lead acid battery model and make it linear in order to ease circuit analysis of the model.

1) The current passes through the parasitic branch $I_{p}$ is around $0.5 \%$ as shown in [19], therefore the parasitic branch will be neglected in the linear model.

2) The time varying component values of the third order model will be averaged over the time to obtain constant component values. 


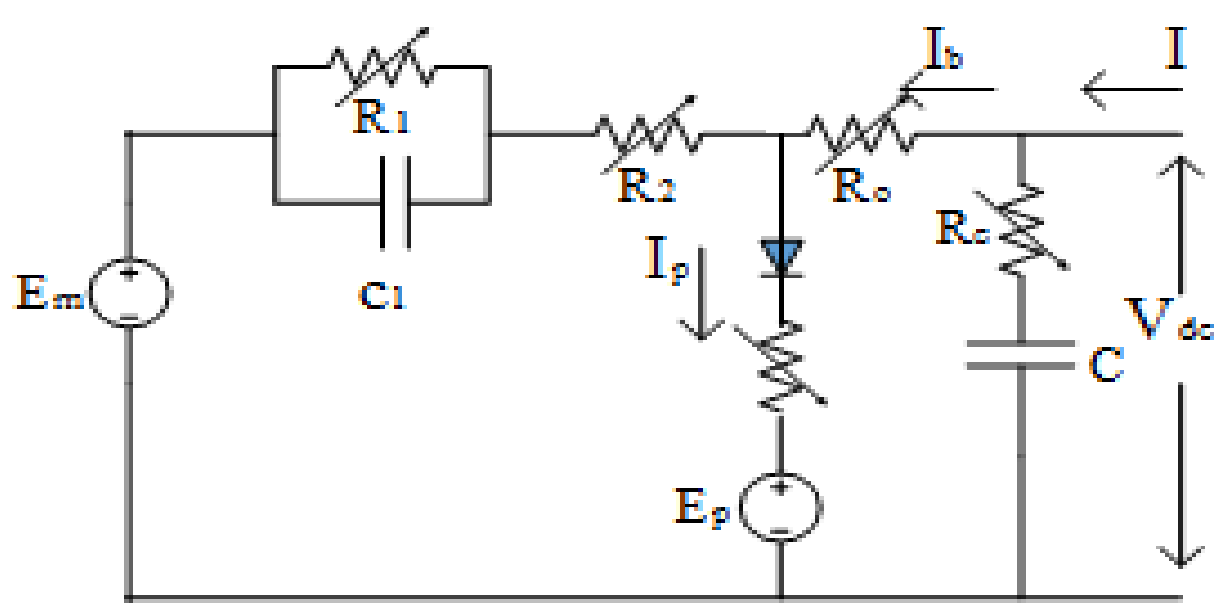

Figure 2.2 Third-order lead-acid battery model [29].

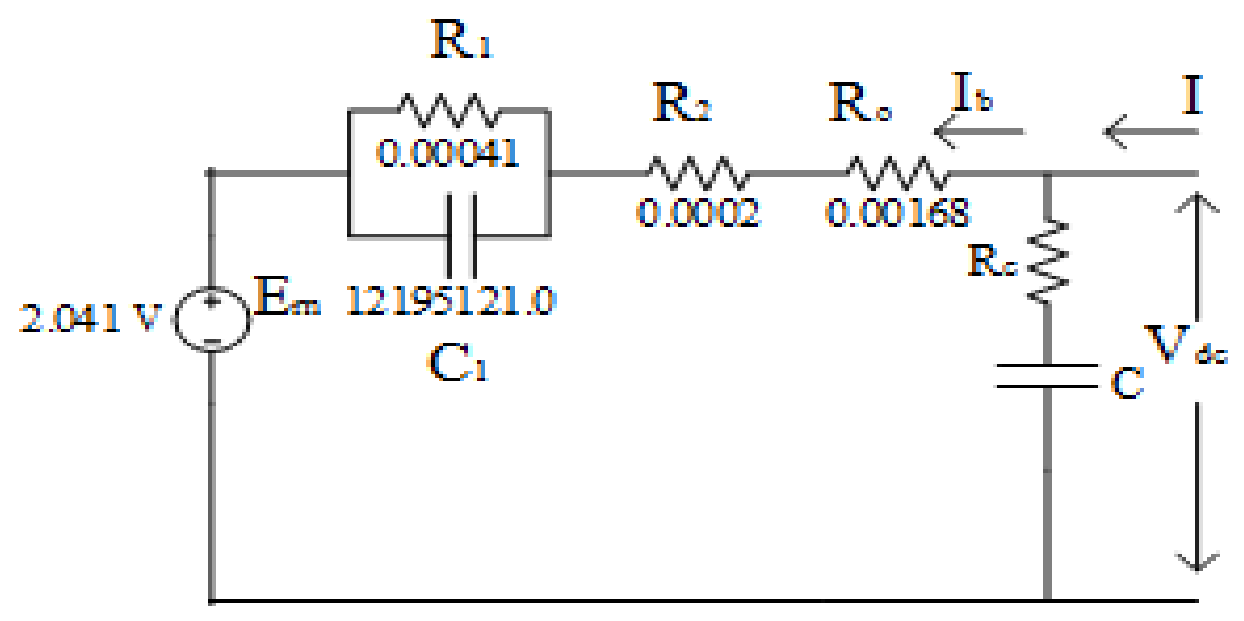

Figure 2.3 Linear battery model [3].

After the simplification of the third order model using the previous mentioned assumptions, the linear model is obtained and it is shown in Figure 2.3. In order to justify the use of this linear model, a simulation for the two models (third order and linear) has been done in [3]. The results of the simulation showed that error in 
calculating SOC, battery input current, and voltage across the battery can be neglected, since it is less than $5 \%$ between the two models.

B) Calculating the DC-link voltage $\mathrm{V}_{\mathrm{dc}}$

The DC-link voltage $\left(V_{d c}\right)$ calculation is done using the linear lead acid battery model shown in Figure 2.3. One can note that, the DC-link voltage $V_{d c}$ is a result of two main factors, the first one is the battery electromagnetic force EMF $\left(E_{m}\right)$ and the other one is the battery terminal input current $(I)$. Since the battery model is linear, the superposition theory can be applied to obtain the value of $V_{d c}$ using basic circuit theories. A complete circuit analysis, with all required details, is done in [24] to calculate the $V_{d c}$ and it has been found that $V_{d c}$ can be written as in Equation (2.6),

$$
v_{d c}(t)=\left[1-\lambda_{1} \exp \left(\frac{-t}{\tau_{2}}\right)\right] E_{m}+\left[\lambda_{2}-\lambda_{3} \exp \left(\frac{-t}{\tau_{1}}\right)+\lambda_{4} \exp \left(\frac{-t}{\tau_{2}}\right)\right] I
$$

Where $\lambda_{1}-\lambda_{4}, \tau_{1}$, and $\tau 2$ are function of the circuit elements.

A numerical procedure to calculate the value of $V_{d c}$ using iterative approach is presented in [24]. This numerical procedure takes into account the varying value of the wind power. The calculation of $V_{d c}$ using this iterative approach can be done using Equations (2.7) and (2.8). 


$$
v_{d c(n)}=\left\{\begin{array}{cc}
v_{d c(1,1)}^{+} & n=1 \\
\sum_{l=1}^{N} v_{d c(l, n)}^{+}+\sum_{l=1}^{N-1} v_{d c(l, n)}^{-} & n=2,3, ., N
\end{array}\right\}
$$

Where $\mathrm{N}$ is the number of time intervals during period $\mathrm{T}, v_{d c(l, n)}^{+}$and $v_{d c(l, n)}^{-}$are given by Equation (2.8):

$$
\begin{aligned}
& v_{d c(l, n)}^{+}=E_{m}-\lambda_{1} E_{m} \exp \left(\frac{-((n-l+1) \Delta t)}{\tau_{2}}\right)+\lambda_{2} I_{l}-\lambda_{3} I_{l} \exp \left(\frac{-((n-l+1) \Delta t)}{\tau_{1}}\right)+ \\
& \lambda_{4} I_{l} \exp \left(\frac{-((n-l+1) \Delta t)}{\tau_{2}}\right) \\
& v_{d c(l, n)}^{-}=-E_{m}+\lambda_{1} E_{m} \exp \left(\frac{-((n-l) \Delta t)}{\tau_{2}}\right)-\lambda_{2} I_{l}+\lambda_{3} I_{l} \exp \left(\frac{-((n-l) \Delta t)}{\tau_{1}}\right)-\lambda_{4} I_{l} \exp \left(\frac{-((n-l) \Delta t)}{\tau_{2}}\right)
\end{aligned}
$$




\subsubsection{State of charge}

The SOC of a battery is defined as the ratio of the battery available capacity with respect to its rated capacity. The SOC of the battery gives a good indication of the time the battery will still be capable to continue working before it needs to be recharged again. If the SOC goes lower than certain limits then the battery will be depleted, on the other hand if SOC goes beyond certain limit then the battery will be overcharged, and in both cases the life time of the battery will be reduced. Therefore, the SOC must be kept between proper upper and lower limits. In [24], the authors did not suggest any procedure to control the SOC, therefore an approach to control SOC is proposed in this work. The target of this control mechanism is to keep SOC between $100 \%$ as an upper limit and $30 \%$ as a lower limit so it does not affect the life time of the battery.

1) In case of SOC goes beyond $100 \%$ then the excess in the power generated from wind farm will be determined and sold to the grid at a cheaper price (e.g. the extra power will be sold for $10 \%$ of its value).

2) In case of SOC goes under 30\% then the BESS will not supply power to the grid (i.e. the dispatched power to the grid will be less than $P_{d}{ }^{*}$ ) and this shortage in the power supplied to the grid will be compensated by other sources (e.g. fuel generator) and also will be penalized in the objective function of the optimization problem, by subtracting the price of this shortage in the dispatched power from the benefit function. 
The calculation of state of charge can be done using Equations (2.9) and (2.10), taken from [29],

$$
S O C=1-\frac{Q_{e}}{K_{c} C_{o^{*}}}
$$

Where $K_{c}$ and $C_{o^{*}}$ are empirical coefficients and $Q_{e}$ is the discharged charge from the battery and it can be found using Equation (2.10),

$$
Q_{e}=\int_{o}^{t}-I(\tau) d \tau
$$




\subsection{Determination of BESS capacity}

The use of previously described procedure to determine the size of BESS will give different BESS size on different days as it depends on the wind power profile which changes daily. However, it is obvious that once the BESS is installed, for a wind farm, its capacity is fixed and it is hard to change it. The challenge then is to find a method to determine the BESS size which is optimal over the planning horizon. In this work, two methods are proposed to determine the size of BESS. The first one is based on calculating the optimal BESS size for every day of the year then creating a probability distribution function of the optimal BESS size. The decision of BESS size then will be taken so that the power can be dispatched at the required level with a certain probability. This will ensure that the BESS can satisfy the needs with a high probability. The other one will be by creating cost function including the cost of power generation using typical fuel generator along with a wind farm connected to BESS then obtaining the BESS size which minimizes the cost function. 


\subsection{Descriptions of methods and results}

In this section the two methods will be described using an example system. In this example, one year (2014) worth of wind speed data averaged over 5-minute intervals is obtained from Abernathy, TX, US to illustrate the BESS design [8]. The wind power profile is calculated from wind speed data using Equation (2.11) taken from [30]. The wind farm capacity is equal to $19 \mathrm{MW}$.

$$
P_{w}=\frac{1}{2} \rho A V^{3} C_{\rho}
$$

Where $\rho$ is air density in $\left(\mathrm{kg} / \mathrm{m}^{3}\right), A$ is the swept area of wind turbine in $\left(\mathrm{m}^{2}\right), V$ is the wind speed in $(\mathrm{m} / \mathrm{s})$, and $C_{\rho}$ is the power coefficient. One year (2014) load data is obtained from Electric Reliability Council of Texas (ERCOT) and this data used in the second method to determine BESS size. The peak load is 62.127 MW and average load is $30.34 \mathrm{MW}$.

\subsubsection{First method: probability of sufficient storage method}

The optimal BESS size needed to dispatch the required level of dispatched power for each day is found using the optimization procedure described earlier. The coefficients ( $\alpha, \beta$, and $\gamma$ ) of Equation (2.5) are taken from [24]. $\alpha=0.065 \$ / \mathrm{kWh}$, $\beta=0.0116 \$ / \mathrm{kW} / \mathrm{h}$ and $\gamma=0.0099 \$ / \mathrm{kWh} / \mathrm{h}$. The frequency distribution for optimal capacity of BESS is created from optimal BESS size for each day and it is shown in Figure 2.4 [31]. The corresponding frequency distribution for the daily dispatched power 
is shown in Figure 2.5 for illustration. The frequency distribution of BESS optimal is then replaced by a probability distribution function (pdf) proposed in the preceding section. A chi-square test is used to verify whether the suggested pdf is a good fit for this frequency distribution. The details of pdf generation and chi-square test are presented in the following discussion.

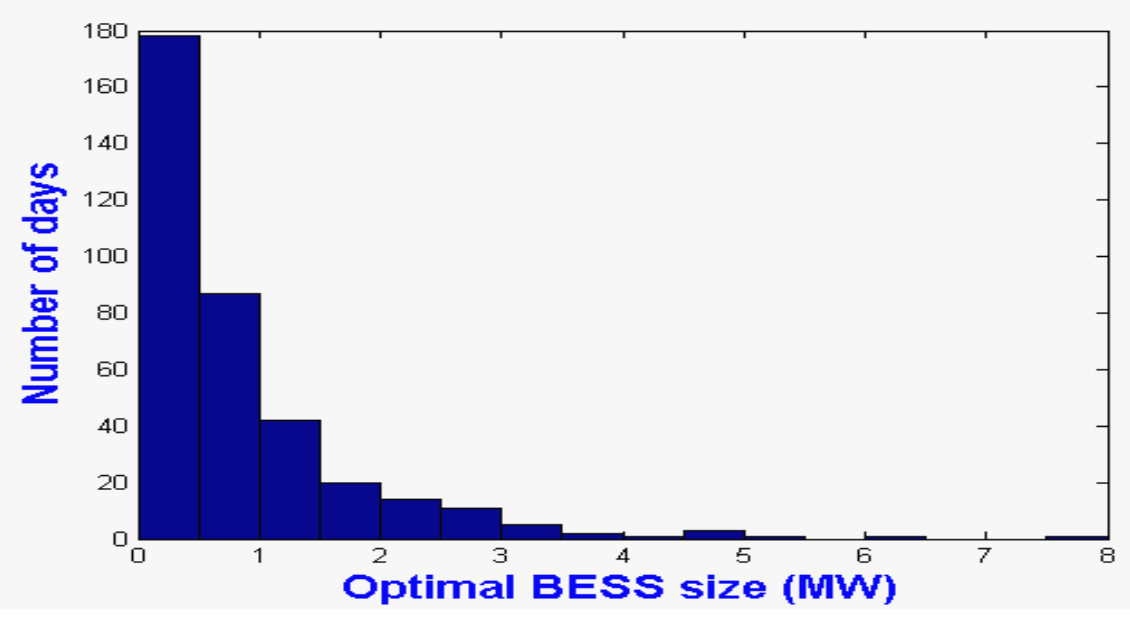

Figure 2.4 Frequency distribution of optimal BESS size.

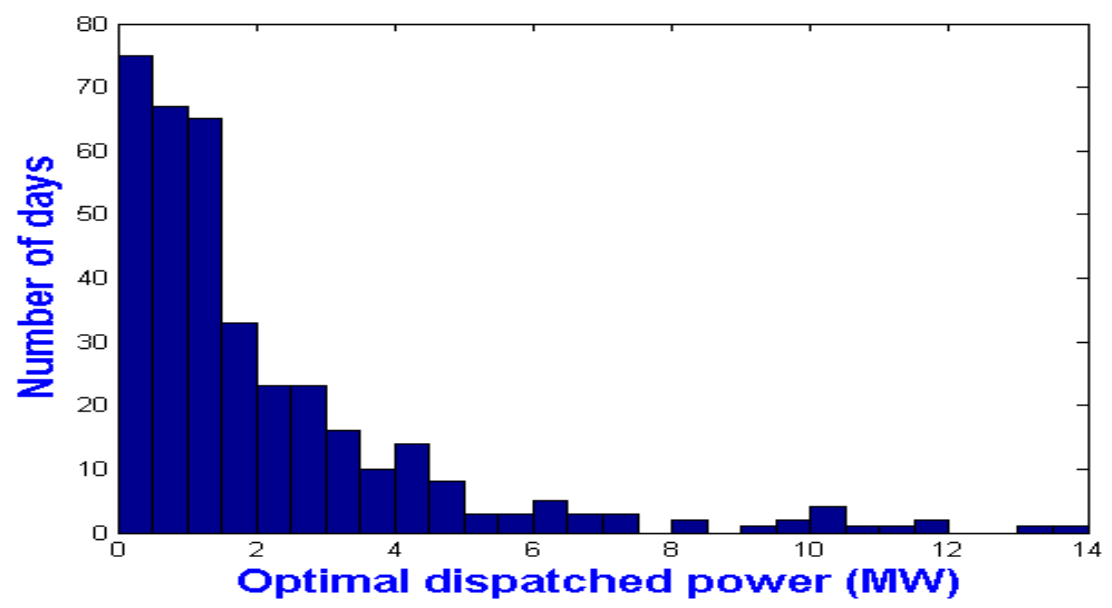

Figure 2.5 Frequency distribution of optimal $P_{d}$ level. 


\subsubsection{Chi-square test}

The frequency distribution of the optimal BESS size, shown in Figure 2.4, is represented by Table number 2.1 .

Table 2.1: Frequency distribution for optimal BESS size

\begin{tabular}{|c|c|c|c|c|}
\hline OS(MW) & $0-0.5$ & $0.5-1$ & $1-1.5$ & $1.5-2$ \\
\hline Freq & 178 & 87 & 42 & 20 \\
\hline OS(MW) & $2-2.5$ & $2.5-3$ & $3-3.5$ & $3.5-4$ \\
\hline Freq & 14 & 11 & 5 & 2 \\
\hline OS(MW) & $4-4.5$ & $4.5-5$ & $5-5.5$ & $5.5-6$ \\
\hline Freq & 1 & 2 & 1 & 0 \\
\hline OS(MW) & $6-6.5$ & $6.5-7$ & $7-7.5$ & $7.5-8$ \\
\hline Freq & 1 & 0 & 0 & 1 \\
\hline
\end{tabular}

In Table 2.1 OS stands for optimal BESS size in MW and freq stands for the number of

days that optimal BESS is found to be needed. A chi-square test is performed in order to verify if this frequency distribution can be replaced by a pdf. From the shape of the frequency distribution, an exponential probability distribution function is suggested to replace the frequency distribution. The pdf of an exponential probability distribution function is shown in Equation (2.12), 


$$
f(x)=\left\{\begin{array}{cc}
\lambda e^{-\lambda x} & x \geq 0 \\
0 & \text { Otherwise }
\end{array}\right\}
$$

Where $x^{1}$ is the mean of the readings. Therefore the sample mean can be used as an estimate of $\lambda$, hence we have $\lambda=\frac{1}{x_{\text {avg }}}$.

Now, $x_{\text {avg }}=\frac{\sum f x}{\sum f}=\frac{317.75}{365}=0.865$, then $\lambda=1.156$.

Thus the suggested pdf in replacement of frequency distribution is given in Equation $(2.13)$

$$
f(x)=1.156 e^{-1.156 x}
$$

Using the characteristics of pdf function the probability of the BESS size to be less than some value $(a)$ is given in Equation (2.14),

$$
P(x \leq a)=\int_{0}^{a} 1.156 e^{-1.156 x} d x=1-e^{-1.156 a}
$$

Now using Equation (2.14), the probabilities and expected frequencies of BESS optimal size values using the pdf are calculated and shown in Table 2.2. 
Table 2.2: The probabilities and expected frequencies using pdf model

\begin{tabular}{|c|c|c|c|c|}
\hline OS(MW) & $0-0.5$ & $0.5-1$ & $1-1.5$ & $1.5-2$ \\
\hline$P$ & 0.439 & 0.2463 & 0.0776 & 0.043 \\
\hline$E$ & 160.2 & 89.9 & 50.4 & 28.3 \\
\hline OS(MW) & $2-2.5$ & $2.5-3$ & $3-3.5$ & $3.5-4$ \\
\hline$P$ & 0.0248 & 0.0142 & 0.007 & 0.0045 \\
\hline$E$ & 15.7 & 9 & 5.2 & 2.6 \\
\hline $\mathrm{OS}(\mathrm{MW})$ & $4-4.5$ & $4.5-5$ & $5-5.5$ & $5.5-6$ \\
\hline$P$ & 0.0025 & 0.0013 & 0.0007 & 0.0005 \\
\hline$E$ & 1.6 & 0.9 & 0.5 & 0.3 \\
\hline $\mathrm{OS}(\mathrm{MW})$ & $6-6.5$ & $6.5-7$ & $7-7.5$ & $7.5-8$ \\
\hline$P$ & 0.0002 & .0001 & 0.0001 & 0.00001 \\
\hline$E$ & 0.2 & 0.1 & 0.05 & 0.05 \\
\hline
\end{tabular}

In Table $2.2 \mathrm{OS}$ is the optimal size, $P$ is the probability obtained from pdf, and $E$ is the expected frequency found by multiplying $\mathrm{P}$ by the total number of samples (i.e. number of days in one year). Applying chi-square test procedure which is require to find the summation of normalized squared error between expected frequencies and real frequencies after adding the classes such that expected frequencies for each class is more than 5 we get data shown in Table 2.3. 
Table 2.3: Normalized error squared for Chi-Square test

\begin{tabular}{|l|l|l|l|}
\hline OS(MW) & $\mathrm{O}_{\mathrm{i}}$ (real frequencies) & $\mathrm{E}_{\mathrm{i}}$ (expected frequencies) & $\frac{\left(O_{i}-E_{i}\right)^{2}}{E_{i}}$ \\
\hline $0-.5$ & 178 & 160.2 & 1.98 \\
\hline $0.5-1$ & 87 & 89.9 & 0.09 \\
\hline $1-1.5$ & 42 & 50.4 & 1.4 \\
\hline $1.5-2$ & 20 & 28.3 & 2.43 \\
\hline $2-2.5$ & 14 & 15.7 & 0.18 \\
\hline $2.5-3$ & 11 & 9 & 0.44 \\
\hline $3-3.5$ & 5 & 5.2 & 0.0077 \\
\hline $3.5-$ inf & 8 & 6.2 & 0.46 \\
\hline
\end{tabular}

Thus, two hypotheses are suggested,

1) $\mathrm{H}_{0}$ : exponential distribution is appropriate fitting.

2) $\mathrm{H}_{1}$ : exponential distribution is not appropriate fitting.

Since the summation of normalized squared error is equal to seven and from tables of chi-square test, for 6 degree of freedom (number of classes - number of conditions) and for $5 \%$ significance level we get $\mathrm{X}_{2}>12.592>7$, therefore, there is no 
evidence at the $5 \%$ significance level to suggest that an exponential distribution is not appropriate.

Once the probability density function is known, the size of the BESS which is good for $90 \%$ of the days, for example, can be found using Equation (2.14),

((i.e., $\left.0.9=1-1.156 e^{-1.156 a}\right)$, This gives $\left.\mathrm{a}=1.99\right)$. So a BESS of size $1.99 \mathrm{MW}$ will be able to be optimum size for $90 \%$ of the days. It should be noted that in this procedure the optimal dispatched power is derived for a given day and then optimal BESS calculated. If more refinement is needed, the optimal dispatched power can be calculated for shorter intervals than a day and then corresponding optimal BESS determined. 


\subsubsection{Second method: cost based method}

The system shown in Figure 2.6 is used to formulate the cost function which then is used in an optimization problem to find the optimal BESS size. In this system, the load is fed from two main power sources which are a wind farm connected to a BESS in addition to a typical fuel generator. The load data taken from ERCOT website [32] and wind farm data is the same as used in the first method. The cost function measuring the total costs related to the BESS size is mathematically formulated in this method. The cost function has two major components which are related directly to the BESS size. The first component represents the BESS installation capital cost as a function of its size. The relationship between BESS capital cost installation and its size, taken from [33], is shown in Figure 2.7. From Figure 2.7, reader can note that the BESS capital cost increases as the BESS size increases which is as expected. The second one measures the cost of the fuel used in the typical fuel generator to meet load requirements. The fuel cost function for fuel generator is a quadratic function of generator output power and it shown in Equation (2.15) [34].

$$
F_{\text {cost }}=a+b^{*} P_{g}+c^{*} P_{g}^{2}
$$

Where $a, b$, and $c$ are the fuel cost coefficients and $P_{g}$ is the power output of the generator. The following values for the coefficients are used: $a=190 \$, b=12 \$ / \mathrm{MW}$ and $c=0.0075 \$ / \mathrm{MW}^{2}$. The fuel cost is found to be influenced by BESS size as shown in the following two different cases: 
1) If wind power is less than BESS size then power generated from the fuel generator will be equal to the difference between load and wind power.

2) If wind power is more than BESS size then power generated from the fuel generator will be equal to the difference between load and BESS size in addition to wind power lost which is equal to difference between wind power and BESS size.

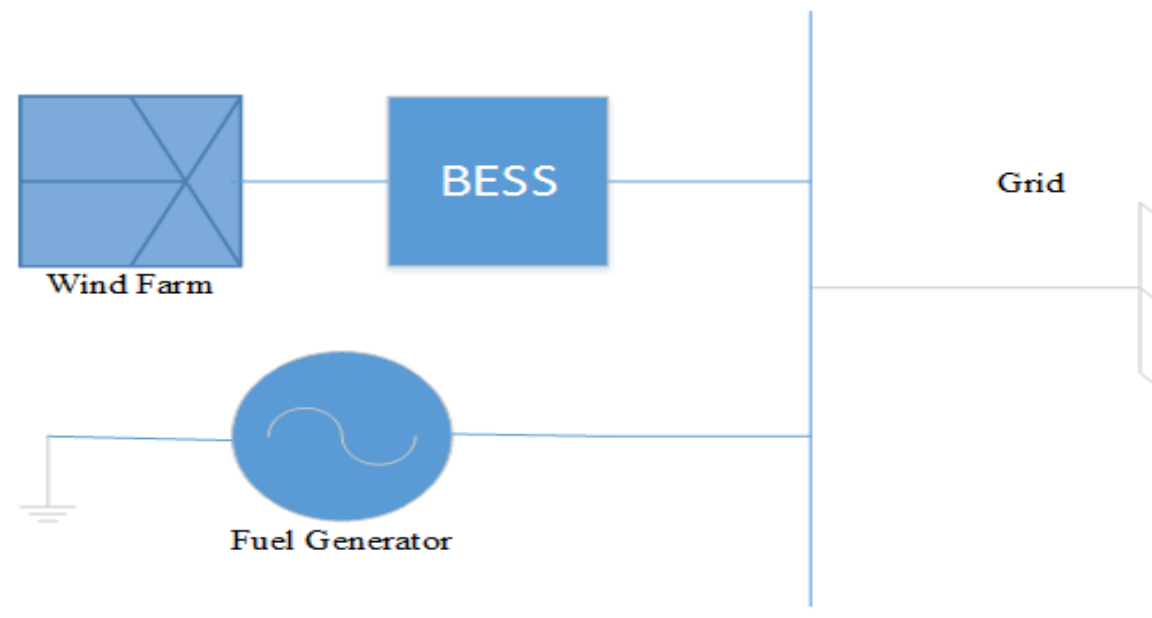

Figure 2.6 System used in the second method.

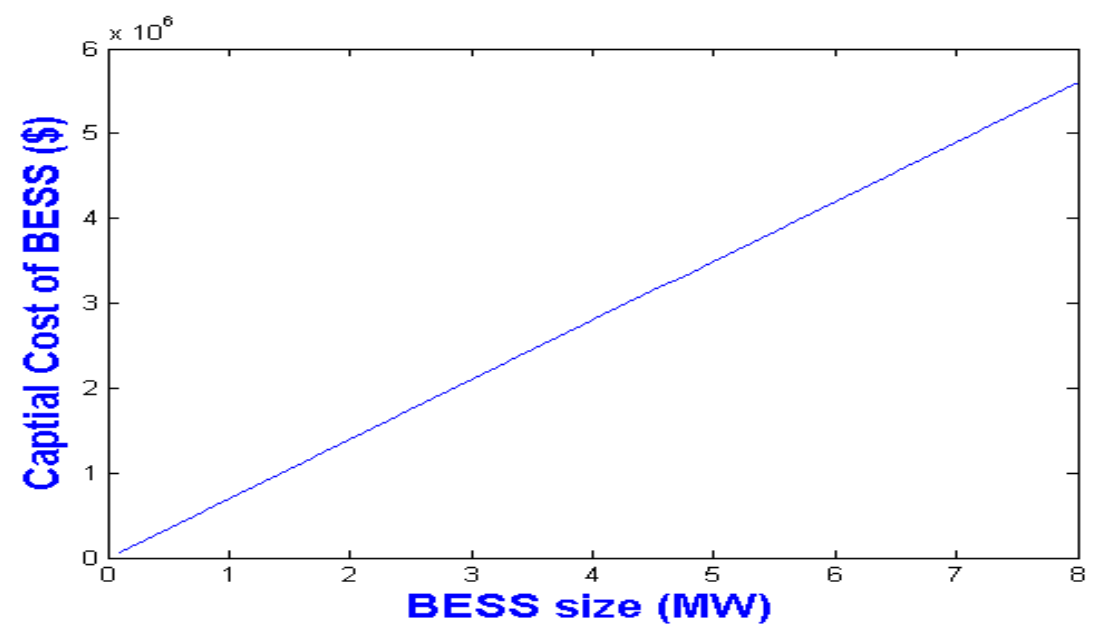

Figure 2.7 Relationship between BESS size and capital cost of BESS in \$. 
The Mathematical formula of the cost function is shown in Equation (2.16),

$$
J=\left\{\begin{array}{cc}
\xi B_{s}+a+b\left(L-P_{w}\right)+c\left(L-P_{w}\right)^{2} & P_{w}<B_{s} \\
\xi B_{s}+a+b\left(L-B_{s}\right)+c\left(L-B_{s}\right)^{2}+a+b\left(P_{w}-B_{s}\right)+c\left(P_{w}-B_{s}\right)^{2} & P_{w} \geq B_{s}
\end{array}\right\}
$$

Where $a, b$ and $c$ are the fuel cost coefficients, $L$ is the Load, $P_{w}$ is the wind power, $B_{s}$ is the BESS size and $\zeta$ is the cost of BESS per kilowatt and equal to $700 \$ / \mathrm{kW}$. The relationship between the second component of cost function and BESS size is shown in Figure 2.8. Total cost against BESS size is shown in Figure 2.9. From Figure 2.9, it is found that optimal size of BESS that minimizes the cost function is equal to $2.1 \mathrm{MW}$.

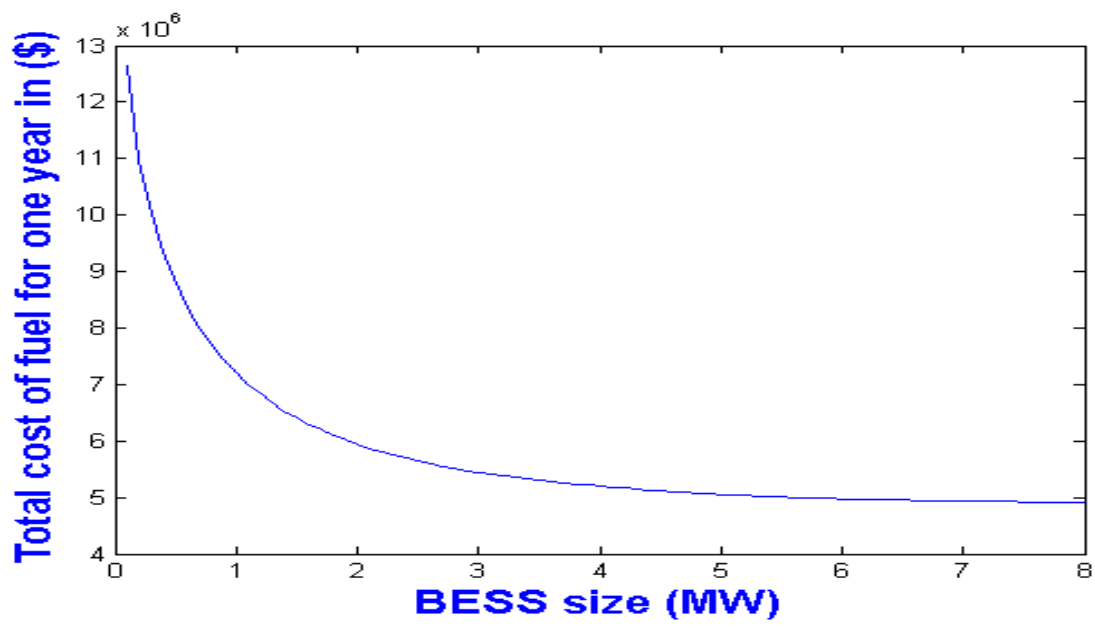

Figure 2.8 Relationship between BESS size and fuel cost per year in $\$$. 


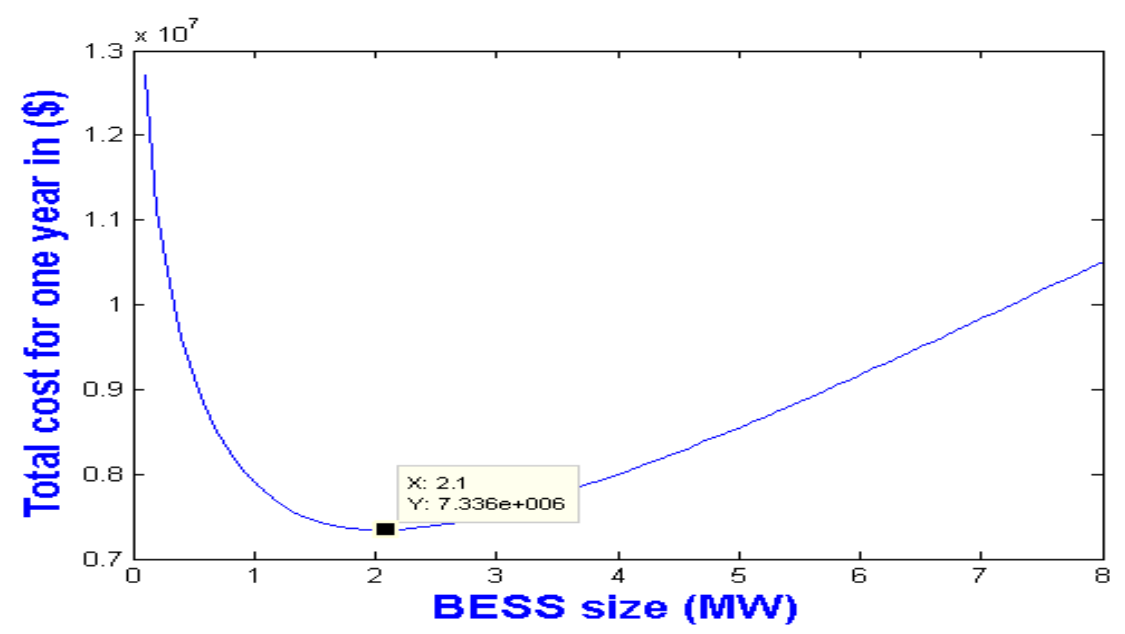

Figure 2.9 Relationship between BESS size and system cost per year in \$.

The optimization procedure developed in the previous sections is summarized by the flow chart shown in Figure 2.10.

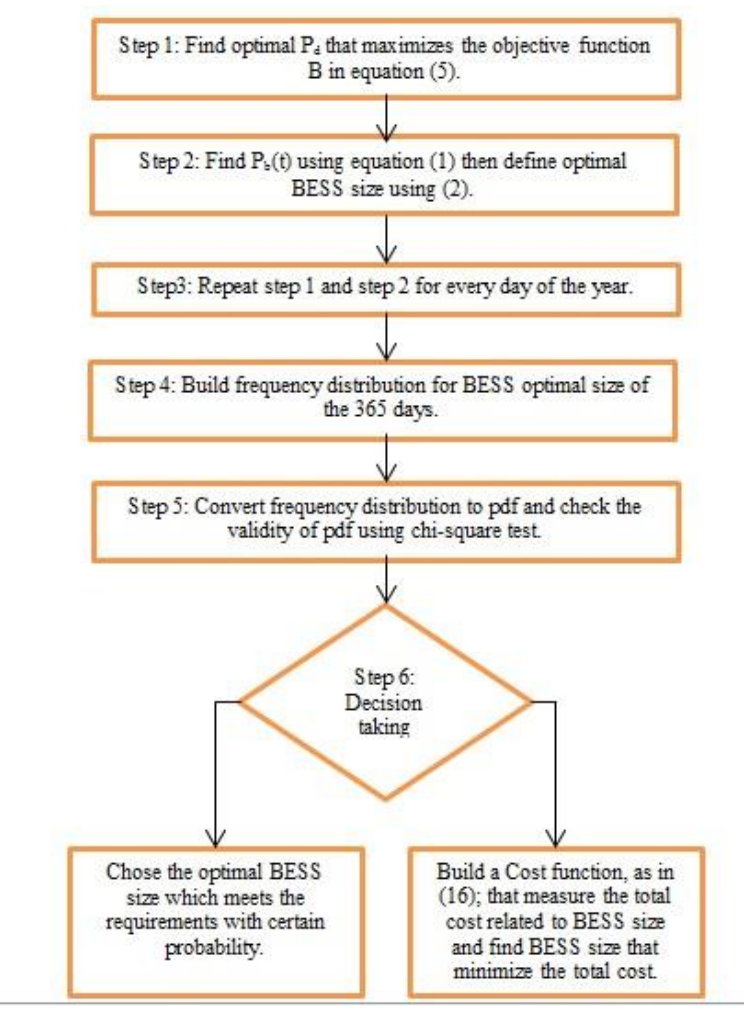

Figure 2.10 Optimization procedure flow chart. 
The verification of meeting constraints requirement can be done by taking one day wind power profile, shown in Figure 2.11, and applying it to the simulation. In Figure 2.12, the BESS power is shown and as expected it is a shifted version of wind power profile by $P_{d}$ optimal which is equal to 0.48 (p.u.) for this day. For SOC constraint, the SOC of the battery, without control strategy, is shown in Figure 2.13 (blue line). The initial state of the battery SOC is considered to be $50 \%$ (for example), and it is observed that SOC goes under the lower limit (i.e. 0.3) at hour 19, because of the shortage in wind power compared to the required level of dispatched power. After applying the control strategy for SOC mentioned earlier, the new SOC is shown in Figure 2.13 (red line) and it satisfies the predetermined thresholds.

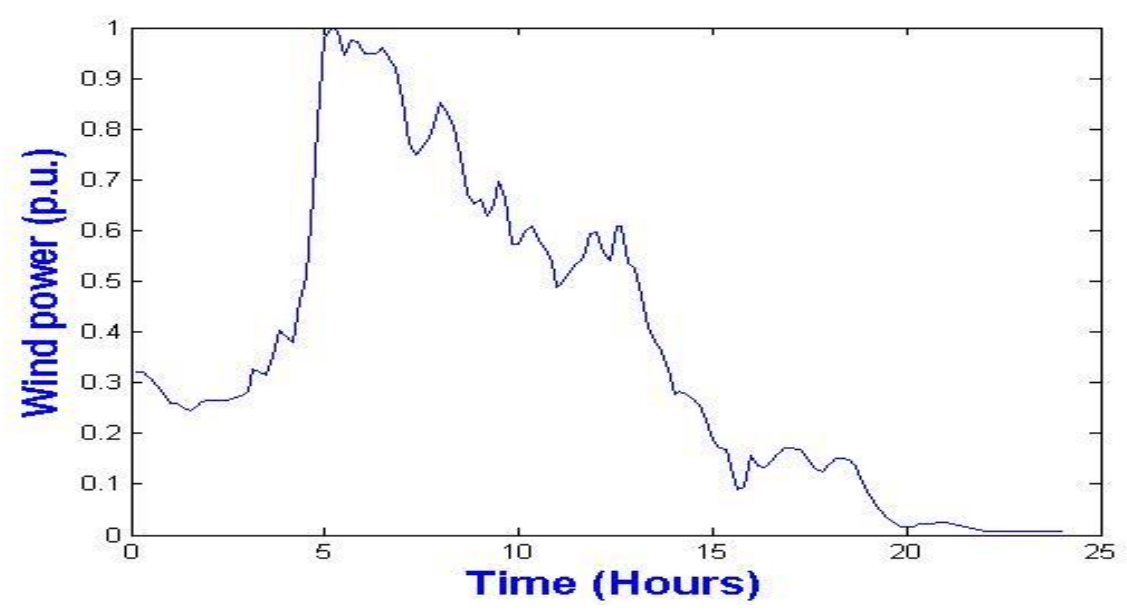

Figure 2.11 Wind power profile for one day. 


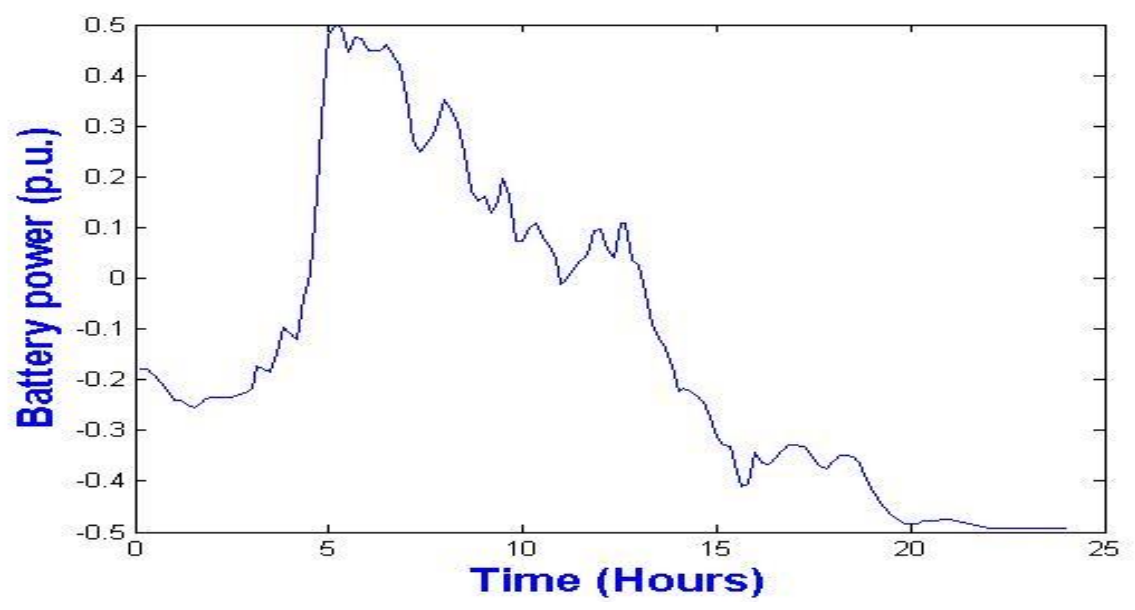

Figure 2.12 Battery power profile.

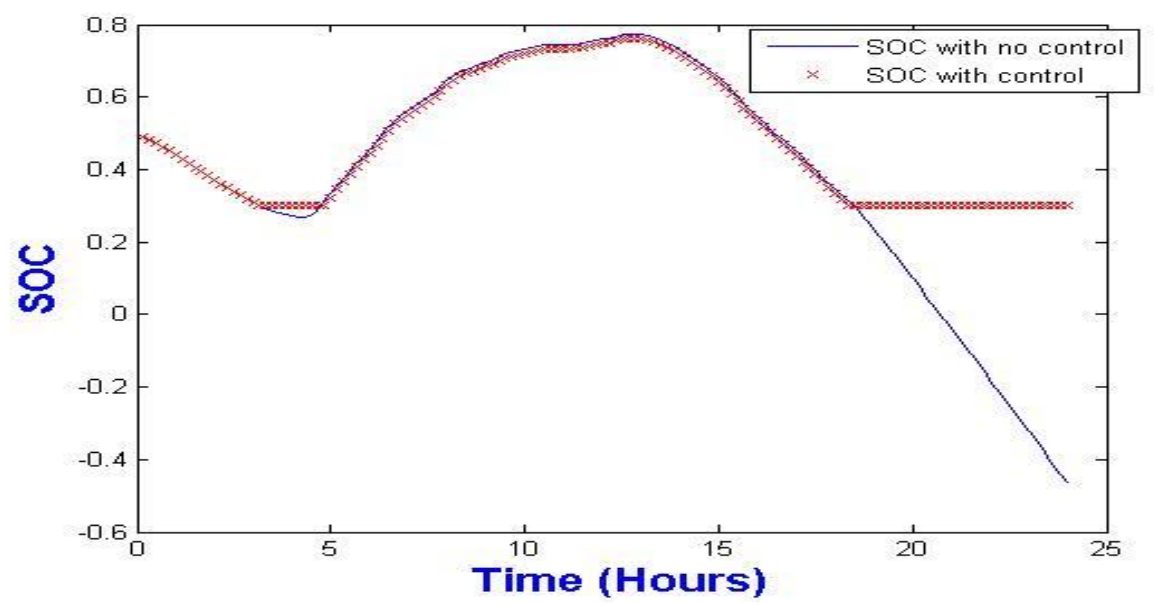

Figure 2.13 SOC of the battery with and without control.

In order to show the effect of the SOC control on BESS power, the BESS power profile after applying SOC control strategy is shown in Figure 2.14. One can note that, it is the same as the battery power profile of no control case before hour 19, since the SOC still above the lower limit then it becomes zero since the SOC is at its lowest level and 
no more power can be supplied from BESS to the grid (same thing happened before hour 5 for short period).

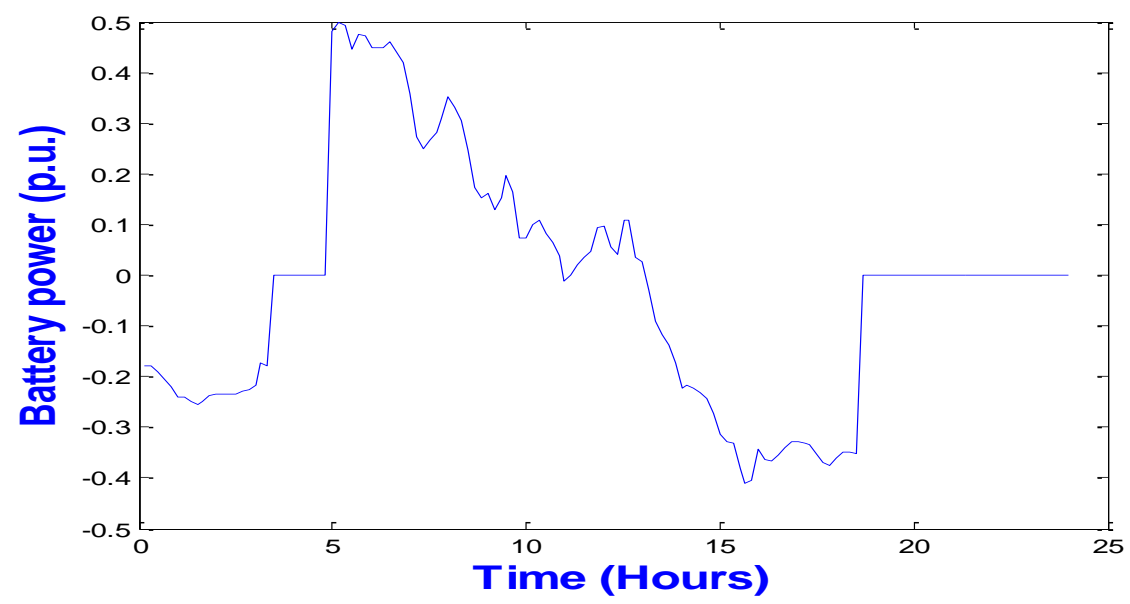

Figure 2.14 Battery power profile with controlled SOC.

The same procedure mentioned in [24] was used to control the DC-link voltage. In Figure 2.15, the DC-link voltage with controlled SOC is shown and it meets the predetermined limits (i.e. between 0.9 and 1.1 (p.u.)).

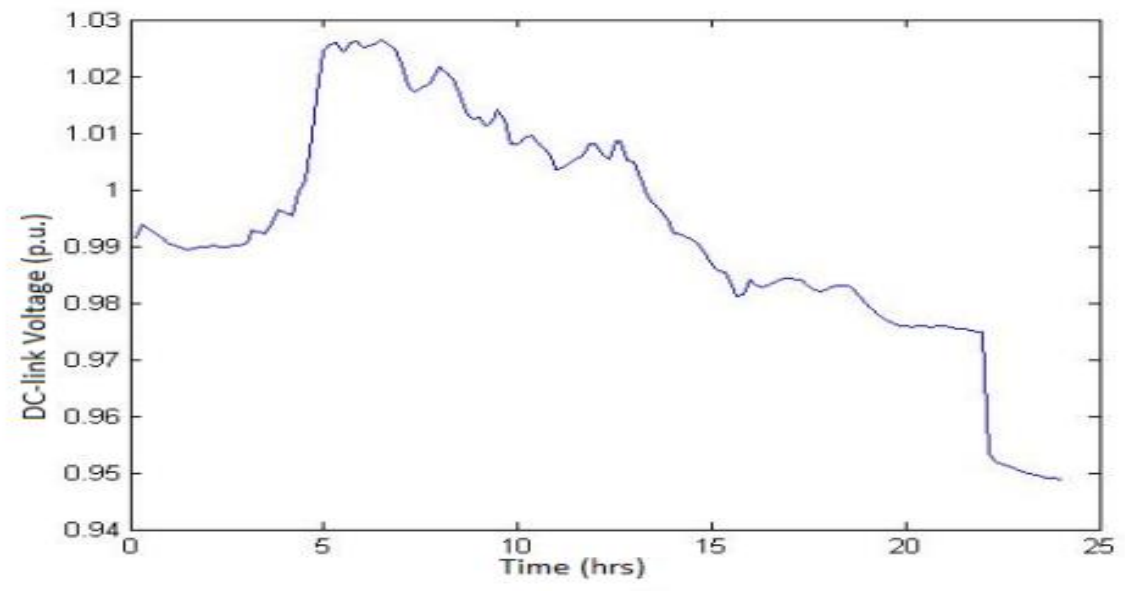

Figure 2.15 DC-link voltage with controlled SOC. 


\subsection{Summary}

The planning of energy storage system for a wind farm, to smooth its output and dispatch power on hourly basis, needs a careful analysis. The optimal size of BESS can be found to be varying due the varying nature of wind power. Therefore a criteria needs to be used to determine the proper size that is applicable over the planning horizon. Two approaches are proposed using different philosophies. One approach suggested is to meet the needs of dispatched power with a certain level of probability or equivalently to be able to do so for a certain percentage of days of the year. This approach is done by finding the frequency distribution of the optimal BESS size for all days of one year. A probability distribution function pdf then will be created to represent the frequency distribution. The validity of this pdf is checked by chi-square test. It should be pointed out, if needed, the optimal size can be based on smaller intervals than a day. The other approach suggested is to develop a cost benefit relationship that measures the cost of complete system with varying BESS size and find the optimal size which minimizes the overall cost. The SOC and DC-link voltage are important constraints in order to guarantee a proper operation for converters and BESS ability to dispatch the required power respectively. 


\section{CHAPTER III}

\section{PLANNING OF A HYBRID ENERGY SYSTEM}

\subsection{Introduction}

Atmospheric pollution due to emission of greenhouse gases has been increasing. Therefore, developed countries made an agreement to reduce these emissions in 2012 by 5.2\% compared with 1990 as stated in the Kyoto Protocol [35]. New approaches towards generating electricity with less use of fossil fuel are thus needed. Hence, renewable resources of energy, such as wind, solar, and hydropower have gained much attention as alternatives for electrical power generation. When properly planned, energy systems which use renewables can not only mitigate the greenhouse effect, but also can be economically efficient. However, renewable sources tend to be intermittent and thus they can be combined with conventional ones to make the overall operation uninterruptable. Hybrid energy systems consist of one or more complementary renewable resources combined with conventional sources like diesel generators [36]. Generally, some form of energy storage is a vital component for most of the current hybrid systems [37].

Renewable energy usage has increased recently, e.g., PV installed capacity increased 60\% between 2004 and 2009 and 80\% in 2011 [38]. Distributed renewable energy sources combined with energy storage can be integrated with point of use energy systems using DC distribution systems [19, 39, 40]. This research effort develops a model for a hybrid energy system with renewable energy sources (PV and wind) combined with energy storage connected through a distribution system. The combination 
of the two types of renewable energy sources is dependent on the local availability of the resources in each geographical area. Therefore, optimal sizing and planning of such a system needs careful analysis by evaluating natural sources available in that area.

The main goal for optimizing the hybrid energy system is to obtain reasonable use of the renewable energies along with the lowest investment in order to satisfy load requirements. In the published literature, different optimization techniques have been used to find the optimal size for such a system. In [41], the authors propose an improved optimal sizing for the hybrid power system in stand-alone and grid connected modes. The optimization is based on higher power supply reliability with optimal battery capacity. Nevertheless, the energy market price has not been considered in the planning criteria. Biogeography Based Optimization (BBO) has been developed to evaluate optimal component sizes of a hybrid power system which reduce overall system cost in [8]. Yet, the correlation between renewable energy production and time is missing. A stochastic framework, using a pattern search-based optimization combined with a sequential Monte Carlo simulation, is developed to find optimal planning of hybrid power system which minimize the system cost and satisfy the reliability requirements [9]. However, the energy price has not been modeled in the proposed system. In [42], a techno economic analysis has been used to find optimal hybrid system configuration by considering different scenarios and choosing scenario with minimum cost while meeting load requirements. But the economic modeling of the system is not explained properly. The feasibility and cost benefit analysis of PV-wind system connected to a conventional grid has been investigated in [43]. In [44], an optimization of the hybrid system using 
wind speed and solar irradiance data is proposed, but the energy constraints are not applied to the sizing procedure. The control and management of the islanded PV-wind and storage systems have been proposed in [40, 45-47]. In [48] and [49], genetic algorithm and neural networks have been used to find optimum sizing for hybrid energy systems.

However, in the previous mentioned studies, the importance of time domain is not considered in the planning of the hybrid energy system. While considering renewable resources, load requirements and energy price are highly related with time. Therefore, an optimization technique using linear programming which includes all these data in time domain is suggested to plan the hybrid energy system. It should be noted that by considering data over sufficiently long time like a year, the uncertainty inherent in the variables and their correlations is included as the time representations of variables represent a realization of the underlying distributions and their correlations. Energy market price data has been included to strengthen the planning of the distributed hybrid energy system. The planning will be more robust by considering energy price which is changing over time, since most renewable resources (wind and solar energy) are also highly correlated with time. Hence, the maximum benefit of using available renewable energy sources can be achieved. 


\subsection{Hybrid energy system description}

The proposed hybrid distribution system, Figure 3.1, consists of wind and solar farms as renewable power sources, energy storage for storing excess energy and to reduce overall system cost by storing energy when it is cheap and discharging during peak energy price. Load profile represents distribution system load data. The energy market is used to purchase required energy needed to satisfy load requirements in case of renewable energy output is not sufficient, thus the overall system reliability increases. Power electronic devices are used to connect system components with the grid. In this chapter $i$ is the index for $i$ 'th day and $j$ is the index for $j$ 'th time step.

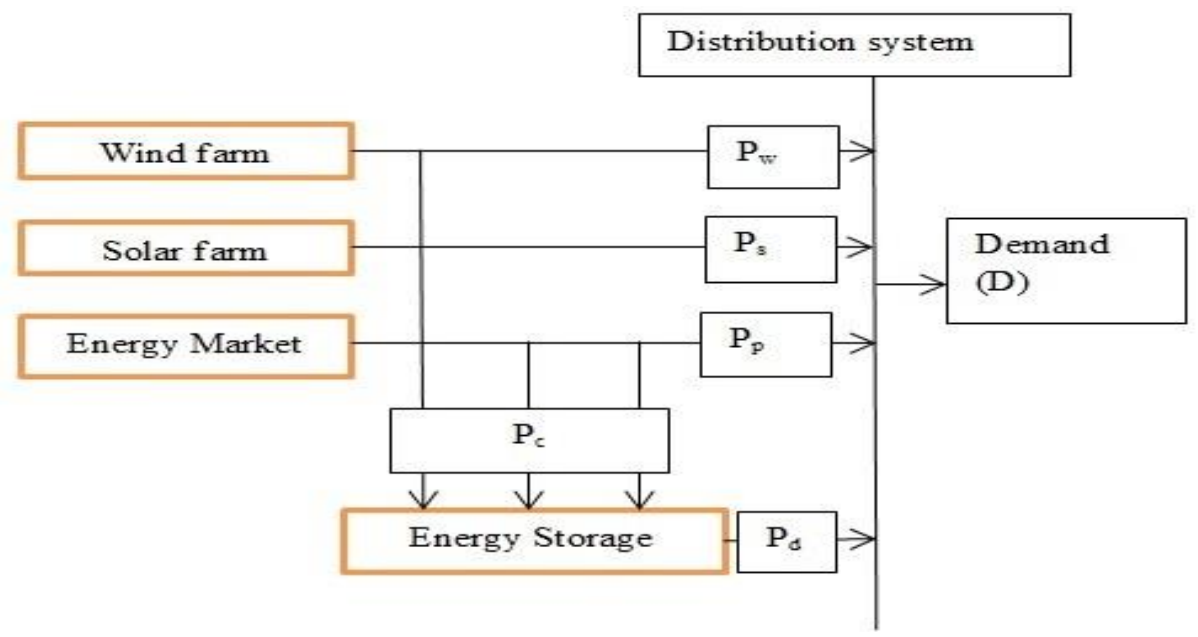

Figure 3.1 Proposed hybrid energy system connected to distribution system.

\subsubsection{Wind farm model}

Cost of energy is typically calculated based on an annual basis, therefore a levelized cost model that converts the total capital cost for a wind farm to present annual 
value is included. The total capital cost of a wind farm which includes turbine system cost and balance of the station cost is levelized over the lifespan of the wind farm. The annual operating and maintenance costs include land lease cost, maintenance cost, and replacement cost. Wind farm size is assumed to be a continuous function, since the capacity of a utility scale wind farm is much larger than turbine rated power. This is a reasonable assumption given that the wind farm is much bigger than a single turbine rating and can ease solving the optimization problem. Electrical power generated from wind turbine depends on wind speed. The relationship between electrical power generated from wind turbine $\left(p_{w i, j}\right)$ and wind speed is shown in Equation 3.1,

$$
p_{w i, j}=\left\{\begin{array}{cc}
0 & v_{i, j} \leq v_{i n} \\
\frac{1}{2} \rho v_{i, j}^{3} \pi \frac{d^{2}}{4 * 10^{6}} C_{\rho} & v_{i n} \leq v_{i, j} \leq v_{r} \\
P_{r} & v_{r} \leq v_{i, j} \leq v_{\text {off }} \\
0 & v_{i, j} \geq v_{\text {off }}
\end{array}\right\}
$$

Where $\rho$ is air density in $\mathrm{Kg} / \mathrm{m}^{3}, v_{i, j}$ is wind speed in $\mathrm{m} / \mathrm{s}, d$ is wind turbine diameter in $\mathrm{m}, C_{\rho}$ is wind turbine efficiency, $v_{i n}$ is cut-in speed for wind turbine in $\mathrm{m} / \mathrm{s}$, $v_{r}$ is rated speed for wind turbine in $\mathrm{m} / \mathrm{s}, v_{\text {off }}$ is cut-off speed for wind turbine in $\mathrm{m} / \mathrm{s}$, and $P_{r}$ is rated power for wind turbine in MW. Thus, the total power generated from wind farm (wind farm utilization) can be calculated using Equations 3.2 and 3.3,

$$
p_{w i, j}=c_{w i, j} * S_{w}
$$




$$
c_{w i, j}=\left\{\begin{array}{cc}
0 & v_{i, j} \leq v_{i n} \\
\left(\frac{v_{i, j}}{v_{r}}\right)^{3} & v_{i n} \leq v_{i, j} \leq v_{r} \\
1 & v_{r} \leq v_{i, j} \leq v_{o f f} \\
0 & v_{i, j} \geq v_{\text {off }}
\end{array}\right\}
$$

Where $c_{w i, j}$ is wind farm utilization factor, and $S_{w}$ is wind farm size in MW.

\subsubsection{Solar farm model}

PV system pricing has gone through rapid changes in the recent years. The cost of PV systems tends to drop with the advancement of PV panel technology. According to the most recent report from the Department of Energy in the United States, the averaged capital cost of PV projects for utility scale solar farm is around $1820 \$ / \mathrm{KW}$ and the total operation and maintenance cost is $12 \$ / \mathrm{KW} /$ year [50]. The electrical power output from PV panel depends on solar radiation. The output power of PV panels can be calculated using Equation 3.4 [51],

$$
p_{s i, j}=s_{i, j} * A_{P V} * \eta_{P V}
$$

Where $p_{s i, j}$ is electrical power generated from PV panel in $\mathrm{W}, s_{i, j}$ is global horizontal irradiance in $\mathrm{W} / \mathrm{m}^{2}, A_{P V}$ is $\mathrm{PV}$ panel area in $\mathrm{m}^{2}$, and $\eta_{P V}$ is efficiency of $\mathrm{PV}$ module. Total solar farm power output (i.e. solar farm utilization) can be found using Equations 3.5 and 3.6, 


$$
\begin{gathered}
p_{s i, j}=c_{s i, j} * S_{s} \\
c_{s i, j}=\frac{s_{i, j}}{s(S T C)}
\end{gathered}
$$

Where $c_{s i, j}$ is solar farm utilization factor, $S_{s}$ is solar farm size in $\mathrm{MW}$, and $s(S T C)$ is global horizontal irradiance at slandered test condition which is $1000 \mathrm{~W} / \mathrm{m}^{2}$.

\subsubsection{Energy storage model}

Lead acid energy storage has been used in the suggested model. Lead acid batteries are the oldest and the most commercially mature form of rechargeable battery technology in the world. Variety of applications like automotive, uninterruptible power supplies, and marine are using lead acid battery technologies. Energy storage in sizes of

10 to $20 \mathrm{MW}$ has been achieved in lead acid carbon technology [6]. Advanced lead acid batteries have been integrated with wind generating sites as in Tappi Wind Park installed in 2001 by Hitachi [5].

Lead-acid life cycle cost analysis is used in this study. The cost estimates are based on capital cost, operation and maintenance cost, and battery replacement cost. Life cycle is estimated with 365 cycles annually for 15 years. The handbook from Sandia Labs describes different lead-acid energy storage systems [5]. The average present value of installed energy storage system cost is $4000 \$ / \mathrm{KW}$ for energy storage power capacity and 400 \$MWh for energy storage energy capacity. 
Power and energy capacities of the energy storage must be defined in order to determine the cost of the system. In the proposed model, the main function of the energy storage is to store energy when it is cheap and discharge the stored energy when energy price is high. However, the power charged/discharged and energy stored must satisfy power and energy capacities given in Equations 3.7 and 3.8,

$$
\begin{gathered}
0 \leq p_{c i, j} \leq P^{*} \\
0 \leq p_{d i, j} \leq P^{*} \\
e_{i, j}=\left\{\begin{array}{cc}
e_{i, j-\Delta t}+\eta_{c} p_{c i, j} \Delta t & p_{c i, j} \geq 0 \\
e_{i, j-\Delta t}-\frac{1}{\eta_{d}} p_{d i, j} \Delta t & p_{d i, j} \geq 0 \\
0 \leq e_{i, j} \leq E^{*}
\end{array}\right.
\end{gathered}
$$

Where $P^{*}$ and $E^{*}$ are power and energy capacities of energy storage, $p_{c i, j}$ and $p_{d i, j}$ are power charged/discharged to/from energy storage, $\eta_{c}$ and $\eta_{d}$ are charging/discharging efficiencies of energy storage, $\Delta t$ is time step, and $e_{i, j}$ is energy stored in energy storage.

\subsubsection{Energy market model}

The energy market is assumed to supply load requirements in the case that power generated from renewable sources is not enough. Incorporating energy market price data into the optimization problem will make the planning of the hybrid energy system more practical, since energy price changes during the day and renewable energy generation is also highly correlated with time. Therefore, maximum benefit from renewables can be 
achieved. The total energy price purchased from energy market can be calculated using Equation 3.9,

$$
\sum_{i} \sum_{j} p_{p i, j} \pi_{i, j} \Delta t
$$

Where $p_{p i, j}$ is power purchased from energy market in $\mathrm{MW}, \pi_{i, j}$ is energy market price in $\$ / \mathrm{MWh}$ and $\Delta t$ is time step in hours.

\subsubsection{Load model}

Distribution system load data is used to create the load profile. The load is fully supplied from the hybrid energy system and the energy market. The load demand at each time step is $d_{i, j}$. 


\subsection{Problem formulation}

In this part, a mathematical formulation of the proposed system will be presented for optimization. The objective is to minimize the annual cost of the overall planned system while satisfying load requirements. The load is assumed to be supplied by the renewable sources and energy market. The result will give the optimal sizes of systems needed to minimize the overall annual cost of the system and meet problem constraints. The optimization is formulated as a linear programming problem as shown in the following discussion.

\subsubsection{Objective function}

Objective function measures the total system annual cost in (\$/year) which includes the initial capital cost and operational and maintenance cost. Major components of the system cost come from wind turbines, PV panels, energy storage, and energy purchased from the market. The capital cost for each component will be levelized over its timespan using Equation 3.10 [52],

$$
\zeta=\frac{\eta(1+\eta)^{N}}{(1+\eta)^{N}-1} * \gamma
$$

Where $\zeta$ is the levelized capital cost in $\$ /$ year, $\eta$ is discount rate of the investment in terms of annual interest per year, $N$ is lifespan of system component in years, and $\gamma$ is capital cost in $\$$. 
From the previous discussion, the objective function can be mathematically formulated as in Equation 3.11,

$$
\min J=\left(\zeta_{w}+\beta_{w}\right) S_{w}+\left(\zeta_{s}+\beta_{s}\right) S_{s}+\zeta_{e 1} P^{*}+\zeta_{e 2} E^{*}+\sum_{i=1}^{365} \sum_{j=1}^{48} p_{p i, j} * \pi_{i, j} * \Delta t
$$

Where $\zeta_{w}$ is levelized total capital wind farm expenditures in $\$ / \mathrm{MW} / \mathrm{year}, \zeta_{s}$ is levelized total capital solar farm expenditures in $\$ / \mathrm{MW} / \mathrm{year}, S_{w}$ is wind farm size in $\mathrm{MW}, S_{s}$ is solar farm size in MW, $\beta_{w}$ is total operational wind farm expenditures in $\$ / \mathrm{MW} /$ year, $\beta_{s}$ is total operational solar farm expenditures in $\$ / \mathrm{MW} /$ year, $\zeta_{e l}$ is levelized capital cost and operational costs for energy storage power capacity in $\$ / \mathrm{MW} / \mathrm{year}, \zeta_{e 2}$ is levelized capital cost and operational costs for energy storage energy capacity in $\$ / \mathrm{MWh} / \mathrm{year}, P^{*}$ and $E^{*}$ are power and energy capacities of energy storage in MW and MWh respectively.

$\sum_{i=1}^{365} \sum_{j=1}^{48} p_{p i, j} * \pi_{i, j} * \Delta t$ is total cost of energy purchased from energy market in $\$ /$ year where $\Delta t^{*} p_{p i, j}$ is energy purchased in MWh and $\pi_{i, j}$ is energy price from the market in $\$ / \mathrm{MWh}$.

\subsubsection{Problem constraints}

1) Power balance to insure that power generated and purchased is sufficient to meet the load demand as shown in Equation 3.12.

$$
P_{w}+P_{s}+P_{p}+P_{d}-P_{c}=D
$$


Where $P_{w}$ is power generated from wind farm for one year in matrix form in MW calculated using Equation 3.2,

$$
\begin{gathered}
P_{w}=C_{w} * S_{w} \\
C_{w}=\left[\begin{array}{ccc}
c_{w 1,1} & \cdots & c_{w 1,48} \\
\vdots & \ddots & \vdots \\
c_{w 365,1} & \cdots & c_{w 365,48}
\end{array}\right]
\end{gathered}
$$

$c_{w i, j}$ is the wind farm utilization factor at day $i$ and time step $j$.

$P_{s}$ is power generated from solar farm for one year in matrix form in MW calculated using Equation 3.5,

$$
\begin{gathered}
P_{s}=C_{s} * S_{s} \\
C_{s}=\left[\begin{array}{ccc}
c_{s 1,1} & \cdots & c_{s 1,48} \\
\vdots & \ddots & \vdots \\
c_{s 365,1} & \cdots & c_{s 365,48}
\end{array}\right]
\end{gathered}
$$

$c_{s i, j}$ is the solar farm utilization factor at day $i$ and time step $j$.

$P_{p}$ is power purchased from energy market for one year in matrix form in MW which can be controlled.

$$
P_{p}=\left[\begin{array}{ccc}
p_{p 1,1} & \cdots & p_{p 1,48} \\
\vdots & \ddots & \vdots \\
p_{p 365,1} & \cdots & p_{p 365,48}
\end{array}\right]
$$


$p_{p i, j}$ is the energy purchased from energy market at day $i$ and time step $j$.

$P_{d}$ is power discharged from energy storage for one year in matrix form in MW which can be controlled.

$$
P_{d}=\left[\begin{array}{ccc}
p_{d 1,1} & \cdots & p_{d 1,48} \\
\vdots & \ddots & \vdots \\
p_{d 365,1} & \cdots & p_{d 365,48}
\end{array}\right]
$$

$p_{d i, j}$ is the power discharged from energy storage at day $i$ and time step $j$.

$P_{c}$ is power charged into energy storage for one year in matrix form in MW which can be controlled.

$$
P_{c}=\left[\begin{array}{ccc}
p_{c 1,1} & \cdots & p_{c 1,48} \\
\vdots & \ddots & \vdots \\
p_{c 365,1} & \cdots & p_{c 365,48}
\end{array}\right]
$$

$p_{c i, j}$ is the power charged into energy storage at day $i$ and time step $j$.

$D$ is load demand for one year in matrix form in MW.

$$
D=\left[\begin{array}{ccc}
d_{1,1} & \cdots & d_{1,48} \\
\vdots & \ddots & \vdots \\
d_{365,1} & \cdots & d_{365,48}
\end{array}\right]
$$

$d_{i, j}$ is the load demand at day $i$ and time step $j$. 


\section{2) Energy storage constraints}

Energy storage has two capacities that limit its operation.

a) Power capacity constraint: power charged/discharged into/from energy storage must be less than its power capacity.

$$
\begin{aligned}
& 0 \leq p_{c i, j} \leq P^{*} \\
& 0 \leq p_{d i, j} \leq P^{*}
\end{aligned}
$$

b) Energy capacity constraint: energy stored into energy storage must be less than its energy capacity.

$$
e_{i, j}=\left\{\begin{array}{cc}
e_{i, j-\Delta t}+\eta_{c} p_{c i, j} \Delta t & p_{c i, j} \geq 0 \\
e_{i, j-\Delta t}-\frac{1}{\eta_{d}} p_{d i, j} \Delta t & p_{d i, j} \geq 0 \\
0 \leq e_{i, j} \leq E^{*} &
\end{array}\right.
$$

From the objective function, it can be noticed that the optimization variables can be classified into two major sets,

1) Decision variables: $S_{w}, S_{s}, P^{*}, E^{*}, P_{p}, P_{c}, P_{d}$ which can be controlled.

2) Given data: $C_{w}, C_{s}, D, \pi$ which are historical data or forecast data based on historical trends.

Total number of decision variables is given in Table 3.1. 
Table 3.1: Decision variables summary

\begin{tabular}{lll}
\hline Decision variable & Variable type & Total number \\
\hline$S_{w}$ & Design & 1 \\
\hline$S_{s}$ & Design & 1 \\
\hline$P^{*}$ & Design & 1 \\
\hline$E^{*}$ & Design & 1 \\
\hline$p_{p i, j}$ & Controlled & $365^{*} 48=17520$ \\
\hline$p_{c i, j}$ & & $365^{*} 48=17520$ \\
\hline$p_{d i, j}$ & Controlled & $365^{*} 48=17520$ \\
\hline
\end{tabular}

Solving the optimization problem gives the optimal wind farm size, solar farm size, energy storage power capacity, and energy storage energy capacity needed to plan the hybrid energy system. Since in practical design there is an area limitation for wind/solar farm which limits the installed capacity of both of them, an upper limit to the design variables (i.e. $S_{w}, S_{s}, P^{*}, E^{*}$ ) can be added into the optimization constraints. 


\subsection{Case study}

The case study is based on a grid tied distribution system in north Texas. The case study employs one year's worth of data for wind speed, global horizontal irradiance, energy market price, and load data in the north Texas area. The wind speed data is taken from the Electric Reliability Council of Texas (ERCOT) for the northern area distribution system [53]. The wind farm cost model is developed based on the 2014 wind technologies market report disseminated by the U.S. Department of Energy (DOE) [54]. The average capital cost for utility scale wind farm projects is $1550 \$ / \mathrm{KW}$, and the annual operating and maintenance cost is $15 \$ / \mathrm{KW} /$ year.

The wind farm uses the NREL wind turbine design cost and scaling model which has the specifications shown in Table 3.2 [55], and is assumed to have a lifespan of 25 years. One year's worth of wind speed data averaged over 30 minute time intervals for the north Texas distribution area is obtained from NREL [27]. The wind speed is measured using anemometer at $50 \mathrm{~m}$ height, therefore the impact of the roughness of the earth's surface on wind speed is found using Equation 3.13,

$$
\left(\frac{v_{i, j}}{v_{o}}\right)=\left(\frac{H}{H_{o}}\right)^{\sigma}
$$

Where $\sigma$ is the friction coefficient, $v_{i, j}$ is wind speed at hub height $(82.5 \mathrm{~m})$ in $\mathrm{m} / \mathrm{s}, v_{o}$ is wind speed at anemometer height $(50 \mathrm{~m})$ in $\mathrm{m} / \mathrm{s}, H$ is turbine hub height $(82.5 \mathrm{~m})$, and $H_{o}$ anemometer height $(50 \mathrm{~m})$. 
The wind farm utilization factor $\left(c_{w i, j}\right)$ for four different days in different seasons, as an example, is shown in Figure 3.2. One can note that wind farm electrical power production is related to time. The wind power during the day time is most likely less than the wind power during the night (i.e. wind speed during night is higher than wind speed during day time).

Table 3.2: Wind turbine characteristics

\begin{tabular}{cc}
\hline Turbine rated power $(\mathrm{MW})$ & 1.91 \\
\hline Turbine rotor diameter $(\mathrm{m})$ & 96.7 \\
\hline Turbine hub height $(\mathrm{m})$ & 82.5 \\
\hline Rotor peak power coefficient $\left(C_{p}\right)$ & 0.47 \\
\hline Cut in wind speed $(\mathrm{m} / \mathrm{s})$ & 2.5 \\
\hline Cut off wind speed $(\mathrm{m} / \mathrm{s})$ & 25.0 \\
\hline Rated wind speed $(\mathrm{m} / \mathrm{s})$ & 9.6 \\
\hline
\end{tabular}

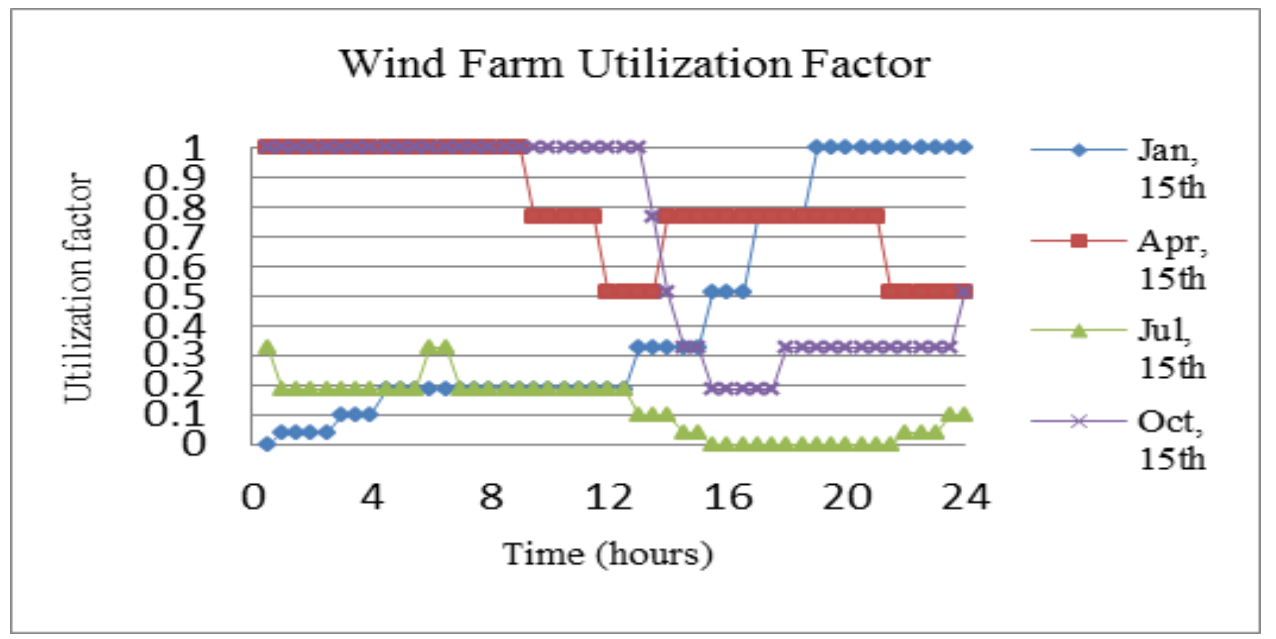

Figure 3.2 Wind farm utilization factor. 
One year of global horizontal irradiance data averaged over 30 minute time intervals is obtained from NREL [27]. The averaged capital cost of PV projects for utility scale is $1820 \$ / \mathrm{KW}$ and the total operation and maintenance cost is $12 \$ / \mathrm{KW} /$ year [50]. The lifespan of the solar farm in this work is assumed to be 33 years, which is around the typical lifespan of solar farm projects. The solar farm utilization factor $\left(c_{s i, j}\right)$ for the same four days is shown in Figure 3.3. The reader can observe that solar farm electrical power production is related to time. Solar power is generated during the day time only, since it depends on solar radiation.

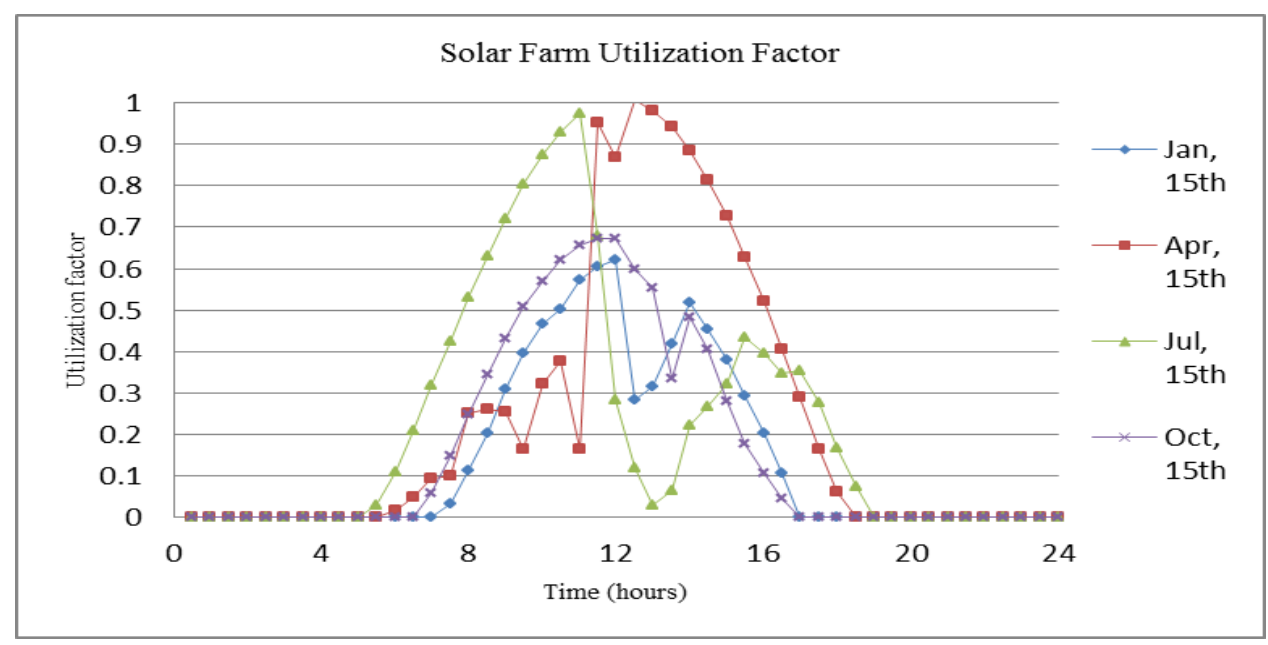

Figure 3.3 Solar farm utilization factor.

Energy market price is collected from ERCOT. The spot market in Texas, where the study is based, is cleared every 15 minute. One year of energy price data is obtained. The energy price for four different days, as an example, is shown in Figure 3.4. The energy price during the day time is most likely more than energy price during the night time. 


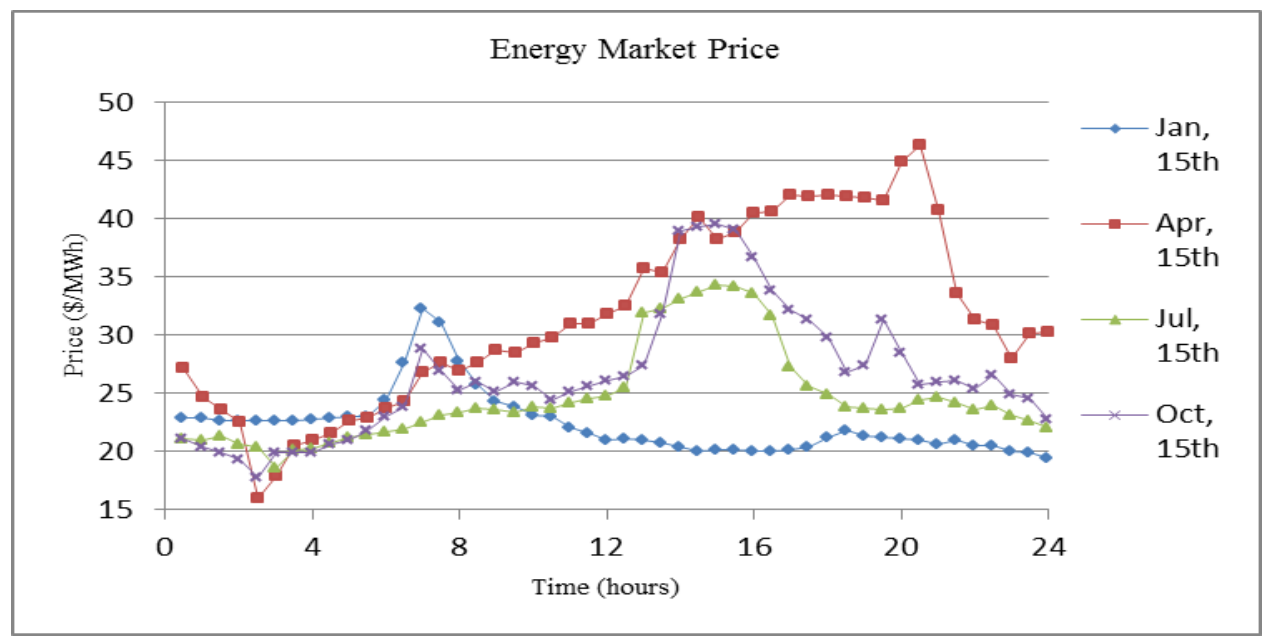

Figure 3.4 Energy price over the time.

The load profile for north Texas distribution system is obtained from ERCOT. One year of load data over one hour time step is obtained. The average load value is 925.2516 MW while the load peak value is 1689.9 MW. Four days load data over the time, for example, is shown in Figure 3.5. The load during the day time is most likely more than the load during the night.

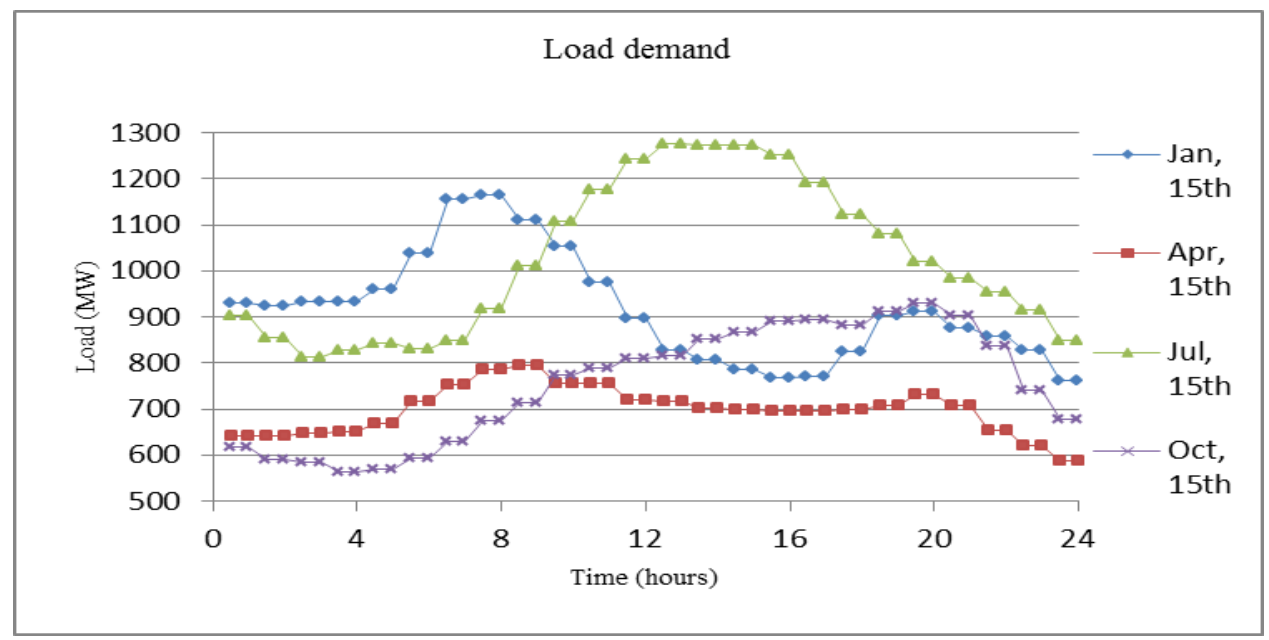

Figure 3.5 Averaged load over the time. 
The simulation time step is 30 minute, therefore all collected data is averaged over 30 minute. System components cost is formulated as mentioned earlier. MATLAB tool box Xprog is used to solve the linear programming optimization problem [56]. The results of case study simulation are given in Table 3.3.

\section{Table 3.3: Results for case study}

\begin{tabular}{ll}
\hline Wind farm size $\left(S_{w}\right)$ & $429.77 \mathrm{MW}$ \\
\hline Solar farm size $\left(S_{s}\right)$ & $38.89 \mathrm{MW}$ \\
\hline Storage power capacity $\left(P_{e}\right)$ & $16.31 \mathrm{MW}$ \\
\hline Storage energy capacity $\left(E_{e}\right)$ & $179.48 \mathrm{MWh}$ \\
\hline Overall system annual cost $(J)$ & $2.6395^{*} 10^{8}$ (\$/year)
\end{tabular}

For this optimal configuration, the levelized cost of energy produced from the wind farm is found to be $27.3882 \$ / \mathrm{MWh}$, and the levelized cost of energy generated from solar farm is $35.0625 \$$ /MWh. The levelized cost of energy for wind farm is less than the levelized cost of energy for solar farm since the wind energy is available throughout the day as compared to solar irradiance which is available during day time only. However, it is still economically efficient to use solar energy since it is highly available during day time at which load and energy price are usually high and wind power generation is low as concluded from the previous discussion.

In case of a system consisting of an energy market only, the annual cost is found to be $2.6422 * 10^{8}$ (\$/year). Thus, the annual cost of the proposed system is less than the 
annual cost of energy purchased from the energy market by only $270 \mathrm{~K} \$$ /year. Although the annual economic benefit from using the proposed system is small as compared to overall annual system cost, it is still encouraging to adopt such a system because it uses clean energy sources. Thus, the traditional ways of generating electrical energy, which cause a great deal of pollution, can be reduced. Furthermore, the wind and solar technologies, used in generating electrical power, are advancing. It is therefore expected that their capital costs will decrease with time as shown in Figures 3.6 and 3.7 [50], [57]. Consequently, the suggested system will be more economically efficient for adoption in the coming years.

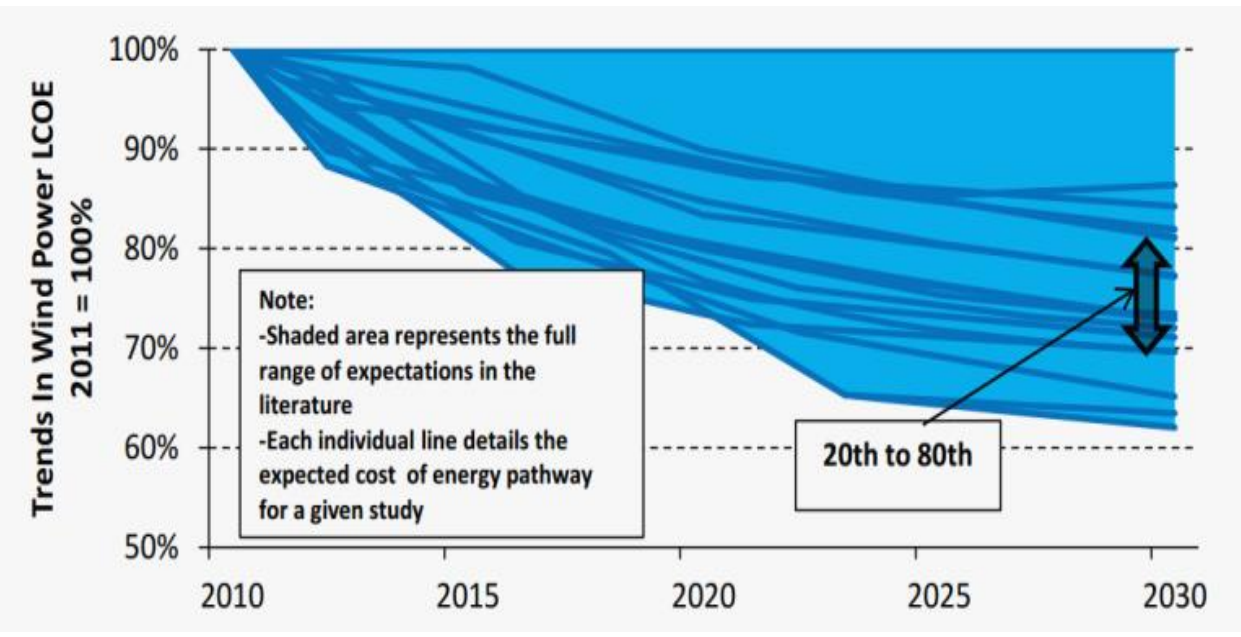

Figure 3.6 Estimated range of wind LCOE projections across 18 scenarios [57]. 


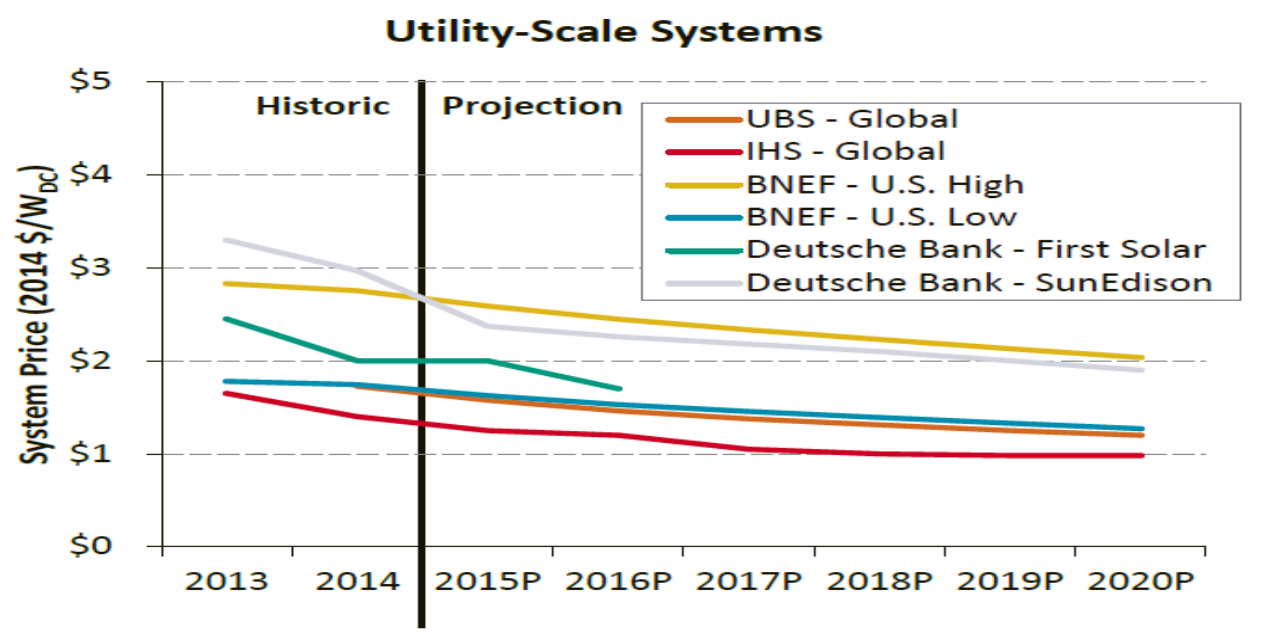

Figure 3.7 Estimated solar farm capital cost projections for utility scale [50].

The energy storage and energy market operation is illustrated in Figures 3.8 through 3.10. It can be noticed that, the power capacity constraint for the energy storage has been satisfied. The energy storage tends to charge power during night time (i.e when energy price and the load are low). On the other hand, the energy storage discharges during day time, since the energy price and load requirements tend to be high. The power purchased from the energy market follows the load demand since power generated from renewable resources is not enough to satisfy the load. 


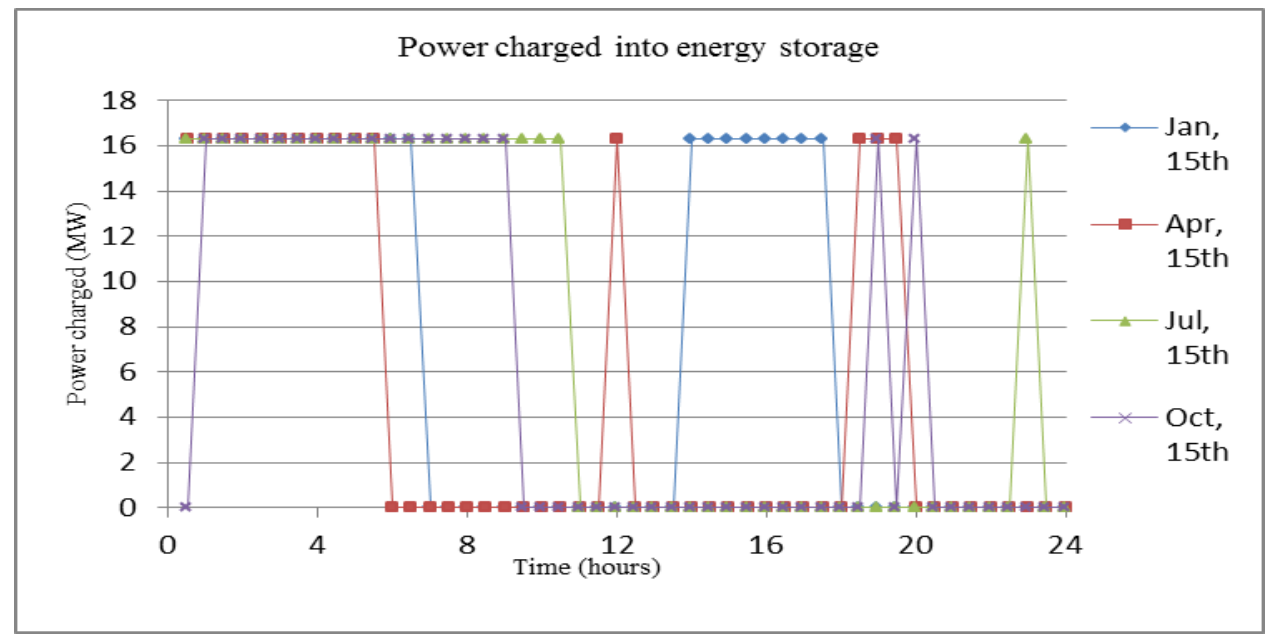

Figure 3.8 Power charged into energy storage.

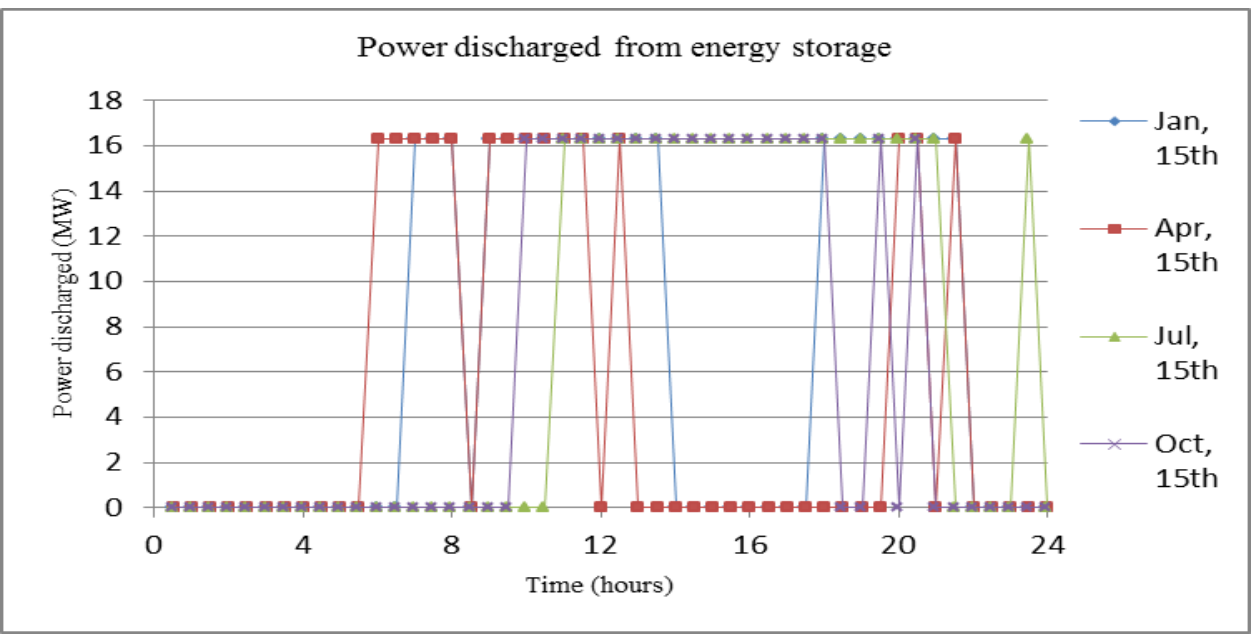

Figure 3.9 Power discharged from energy storage. 


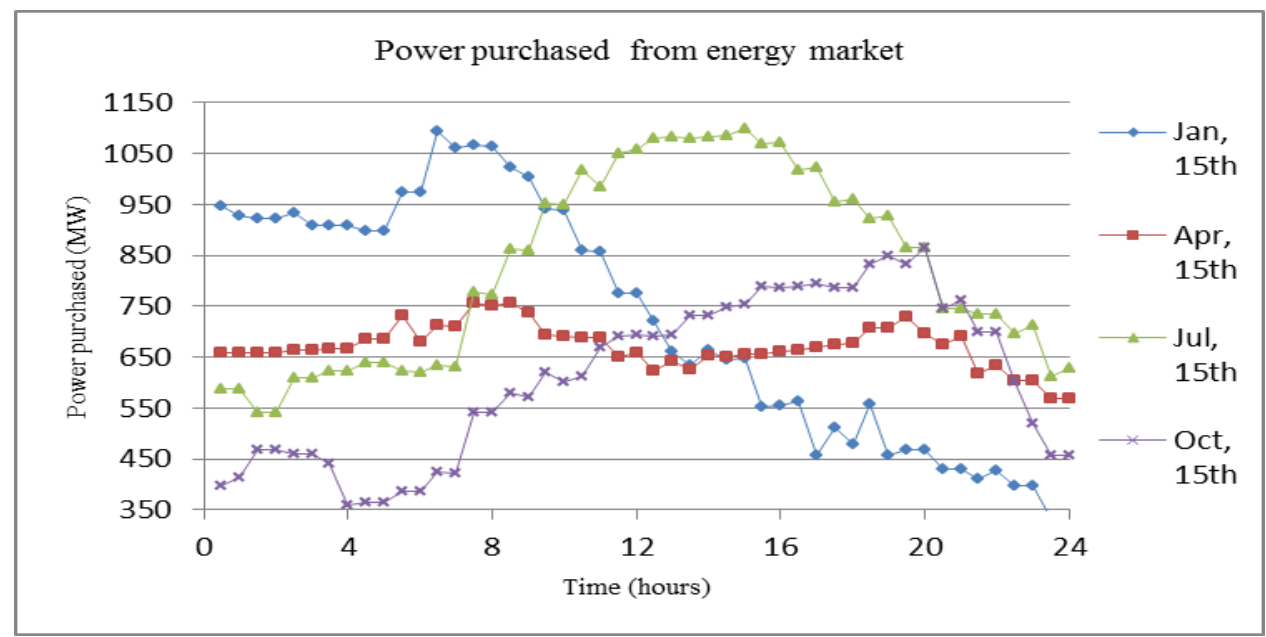

Figure 3.10 Power purchased from energy market.

\subsubsection{Sensitivity analysis}

In this study, four scenarios for sensitivity analysis are considered. In the first one, the load demand is changed between $80 \%$ and $120 \%$ of its value. For the second one, four hours of peak load are increased by $10 \%$ to $30 \%$ (i.e. load profile is made more sharp) then four hours of off peak load are increased by the same amount (i.e. load profile is more flat). For the third one, the hybrid energy system is assumed to have either wind farm or solar farm as the only renewable resource with energy storage. In the fourth scenario, the hybrid system will have renewable sources only without storage. The four scenarios are studied using the same optimization procedure described earlier with some modifications to satisfy each scenario condition. The effect of load changing on the system design is illustrated in Figures 3.11 through 3.14 , the results for scenario two are given in Table 3.4 and the results for scenarios two and three are given in Table 3.5 . 


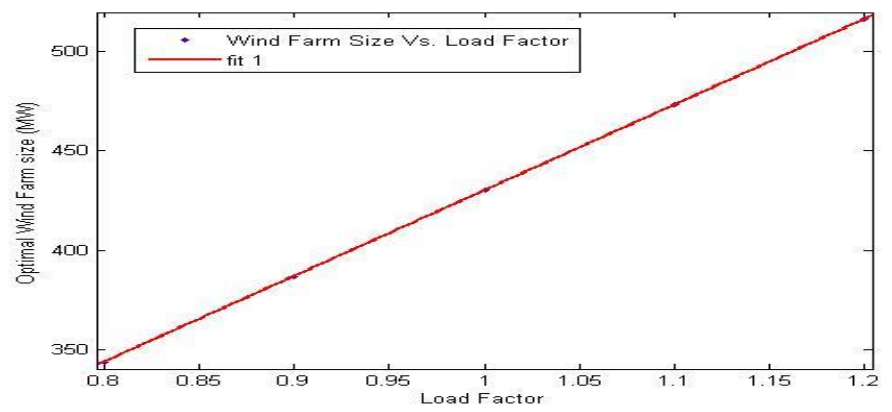

Figure 3.11 Optimal wind farm size Vs. load factor.

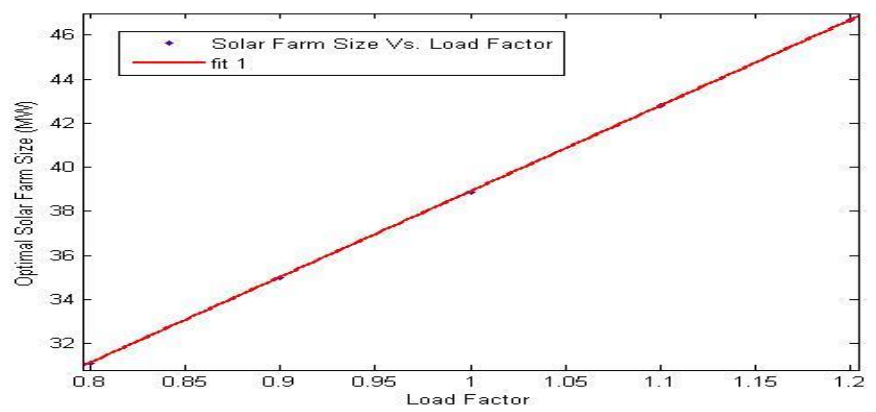

Figure 3.12 Optimal solar farm size Vs. load factor.

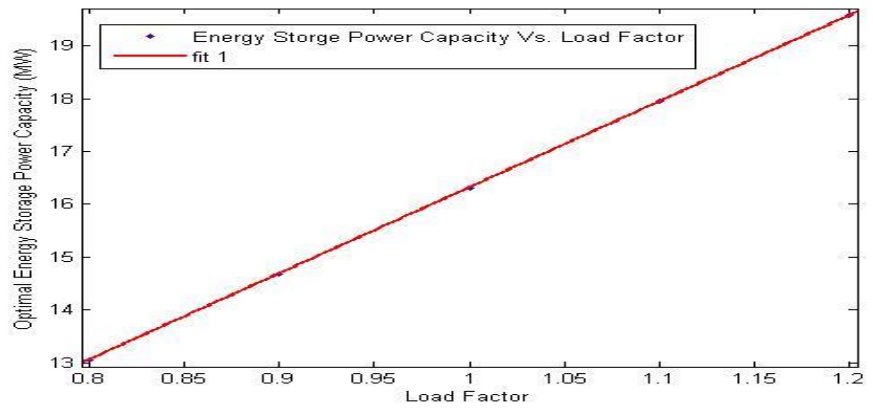

Figure 3.13 Optimal energy storage power capacity Vs. load factor. 


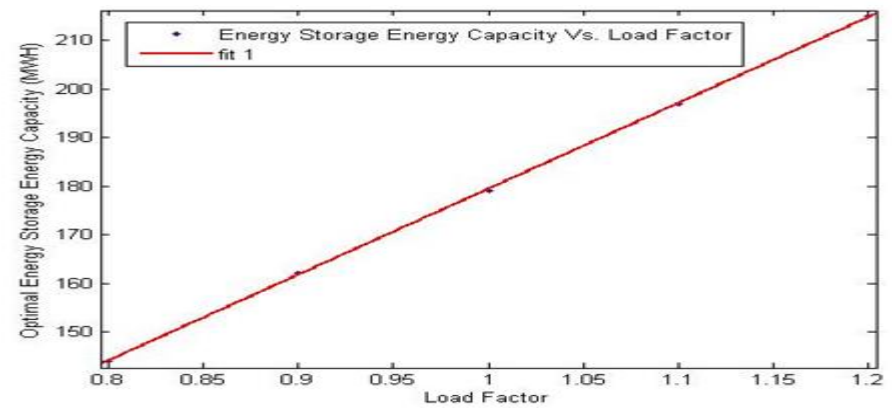

Figure 3.14 Optimal energy storage energy capacity Vs. load factor.

Table 3.4: Results for scenarios two

\begin{tabular}{|l|l|l|l|l|l|}
\hline $\mathrm{D}_{\max }+\%$ & $S_{w}(\mathrm{MW})$ & $S_{s}(\mathrm{MW})$ & $P_{e}(\mathrm{MW})$ & $E_{e}(\mathrm{MWH})$ & $J(\mathrm{~m} \$ / \mathrm{yr})$ \\
\hline $10 \%$ & 465.5 & 50.5 & 17.65 & 194.2 & 270 \\
\hline $20 \%$ & 465.3 & 101.1 & 17.53 & 192.9 & 278 \\
\hline $30 \%$ & 465.1 & 125.3 & 17.42 & 191.6 & 285 \\
\hline $\mathrm{D}_{\min }+\%$ & $S_{w}$ & $S_{s}$ & $P_{e}$ & $E_{e}$ & $J$ \\
\hline $10 \%$ & 429.7 & 38.89 & 16.31 & 179.48 & 266 \\
\hline $20 \%$ & 429.7 & 38.89 & 16.31 & 179.48 & 268 \\
\hline $30 \%$ & 429.7 & 38.89 & 16.31 & 179.48 & 271 \\
\hline
\end{tabular}


Table 3.5: Results for scenarios three and four

\begin{tabular}{llll}
\hline Scenario & Wind farm only & Solar farm only & Without storage \\
\hline Wind farm size (MW) & 430.51 & 0.0 & 26.77 \\
\hline Solar farm size (MW) & 0.0 & 482.23 & 460.10 \\
\hline Storage power capacity (MW) & 16.34 & 0.0 & 0.0 \\
\hline Energy capacity (MWh) & 179.81 & 0.0 & 0.0 \\
\hline Annual cost (\$/year) & $2.6396 * 10^{8}$ & $2.6419 * 10^{8}$ & $2.6397 * 10^{8}$ \\
\hline$\%$ Annual increase & $0.0038 \%$ & $0.0909 \%$ & $0.0076 \%$ \\
\hline
\end{tabular}

Some observations can be made from sensitivity analysis. As load demand changes, the optimal sizes for hybrid system components change proportionally in a linear fashion. However, when the peak load increased the solar farm size increased drastically. This can be justified by the fact that the peak load is usually during the day time at which the solar energy is highly available. On the other hand, when the off peak load increased there was no change in the original optimal system, since the change in the demand is slight and the original system is already capable to meet this slight change. In case wind farm is the only available renewable resource, the optimal size for system components has not changed much, since the solar farm size is small compared to wind farm size in the original system. On the other hand, when solar farm is the only available renewable resource, the optimal size for solar farm has increased dramatically to meet load demand, since it is the only available renewable resource and to compensate wind farm power generation in the original system. On the other hand, energy storage is 
not needed anymore, since the solar irradiance is available during day time only at which the load demand and energy price are high, thus there is no benefit to store energy. When the energy storage is not included in the hybrid system, wind farm size has considerably declined and solar farm size has increased. This can be justified by considering the fact that, wind energy production is high when the load demand and energy price are low and this energy cannot be stored, while the solar farm size increased to substitute the shortage in energy production from wind farm. 


\subsection{Summary}

A general hybrid energy system planning model has been proposed to consider cost of renewables, energy storage and energy market price. An important feature is a hybrid energy system sizing model that maximizes the benefit from using renewable sources which are highly correlated with time by considering real time energy market price which changes with time as well. Thus the hybrid system planning will be more robust and able to take maximum advantage of available renewable resources.

In this model wind speed, solar irradiance, energy market price, and load data are used to optimize hybrid energy system planning using linear programming. The linear programming is used to get values of design parameters which will meet load requirements, practical problem constraints, and reduce overall annual system cost. A case study has been conducted using real data of north Texas distribution system to illustrate the feasibility of the suggested system. Sensitivity analysis which considers changes in load demand and hybrid system configuration has been performed. From the sensitivity analysis, a strong relationship between wind farm and energy storage is indicated.

Costs for wind and PV farms have declined over the recent years and this phenomenon is expected to continue, because of the advancement in the technology used in generating electrical power from renewable resources. This work shows that it is efficient to use renewable energy system even from economical point of view. Therefore, in the next few years this topic will gain more importance since the energy 
generated from renewable resources will be more competitive with current traditional air polluting ways to generate energy. 


\section{CHAPTER IV}

\section{PLANNING OF A HYBRID WIND-PV DISTRIBUTION SYSTEM CONSIDERING SYSTEM UNCERTAINTIES}

\subsection{Introduction}

Traditional ways of electricity generation which use fossil fuel are a major contributor of atmospheric emissions. New approaches towards generating electricity using clean sources of energy, such as wind, solar, and hydropower are thus needed. With proper planning of hybrid energy systems which use renewables, hybrid systems can mitigate the greenhouse effect, and be economically efficient as well. However, renewable sources are intermittent and unpredictable thus conventional generators, like diesel generators, can be added to the system in order to make the overall operation reliable.

Hybrid energy systems combine conventional sources with one or more renewable resources [36]. Generally, energy storage is usually considered as a vital component for most of the hybrid systems [37].

Renewable energy installed capacity has increased recently, e.g., PV usage increased $60 \%$ between 2004 and 2009 and $80 \%$ in 2011 [38]. The present study proposes a planning procedure for a hybrid energy systems which use wind and PV combined with energy storage connected through a distribution system. 
The proposed procedure considers system cost, uncertainties, and reliability in the planning procedure. Renewable energy sources are dependent on the local availability of the renewable resources in the geographical area (wind energy depends on wind speed and solar energy depends on solar radiation). Therefore, natural sources availability in that area should be carefully considered in the planning of such a system.

Optimizing and planning of the hybrid energy system aims to obtain reasonable use of the renewable resources in order to satisfy lowest investment, load requirements, system uncertainties and system reliability. However, planning such a system, which has large number of random variables, makes its optimal configuration more difficult [58]. Cost benefit analysis to find the financial feasibility of PV-wind hybrid energy system connected to a grid has been investigated in [43]. In [40, 45-47], the control and management of the islanded hybrid energy systems have been proposed.

In the published literature, many optimization procedures have been developed to find the optimal planning for such a system. In [8], the authors develop a procedure to find optimal planning for hybrid energy system which reduces overall system cost using Biogeography Based Optimization (BBO). Yet, the time correlation between renewable energy sources has not been considered. In [44], wind speed and solar irradiance data have been used to find optimal configuration of the hybrid system, but the energy storage constraints are not applied to the sizing procedure. Wind speed and solar irradiance data have been used to find optimal configuration of the hybrid system, but the energy storage constraints are not applied to the sizing procedure. In [41], an improved optimal sizing for the hybrid power system has been proposed for both stand- 
alone and grid connected modes. The optimization uses optimal battery capacity and higher power supply reliability. Nevertheless, the uncertainties of renewable resources and the reliability of system components are not applied to system planning. In [59, 60], optimization procedures are developed to plan and control the hybrid energy system. However, the reliability of a system for the grid independent applications is not evaluated. The authors of [9] suggest a pattern search-based optimization combined with a sequential Monte Carlo Simulation to plan hybrid power system which minimizes the system cost and satisfy the reliability requirements. However, the time correlation between renewables has not been covered considering that all of hybrid energy system components are highly related with time. In [42], different scenarios have been considered and the scenario which meets load requirements with minimum cost has been chosen using a techno economic analysis. But the system uncertainties and components reliability have not been modeled.

However, in the previous mentioned and other different studies, the importance of the time correlation between system components (wind speed, solar radiation, energy market price, and load) is not considered in the planning of the hybrid energy system. While considering renewable resources, load requirements and energy price are highly related with time. The uncertainties in wind speed, solar radiation, energy market price and load as well as hardware components reliability are also important in designing such a system. Therefore, an optimization technique which includes time correlated distributions of system uncertainties and adequate models for system components reliability is proposed. By considering time correlation and components reliability, the 
planning will be more robust, since most renewable resources (wind and solar energy), load, and energy price are also highly correlated with time. Hence, the maximum benefit of using available renewable energy sources can be achieved.

In this research effort, two stochastic optimization procedures are suggested to find optimal plan of hybrid energy system.

1) System planning using two-stage stochastic programming.

2) System planning using scenario aggregation procedure. 


\subsection{Hybrid energy system description and subsystem models}

This section provides an explanation of modeling of distribution system components which are the renewable resources, energy market, energy storage and load. The suggested hybrid distribution system, shown in Figure 4.1, has the following components. Renewable power sources in form of wind and solar farms. The role of energy storage is to store the surplus of energy generated from the renewables and to lower system cost by charging when energy price is cheap and supplying the stored energy when the load or the energy price are high. Load profile represents the required demand of the distribution system. The energy market is used to purchase energy needed to fulfill the system requirements in case of renewable energy generation is not sufficient or the price of the energy is lower than the levelized cost of energy (LCOE) generated from the renewables.

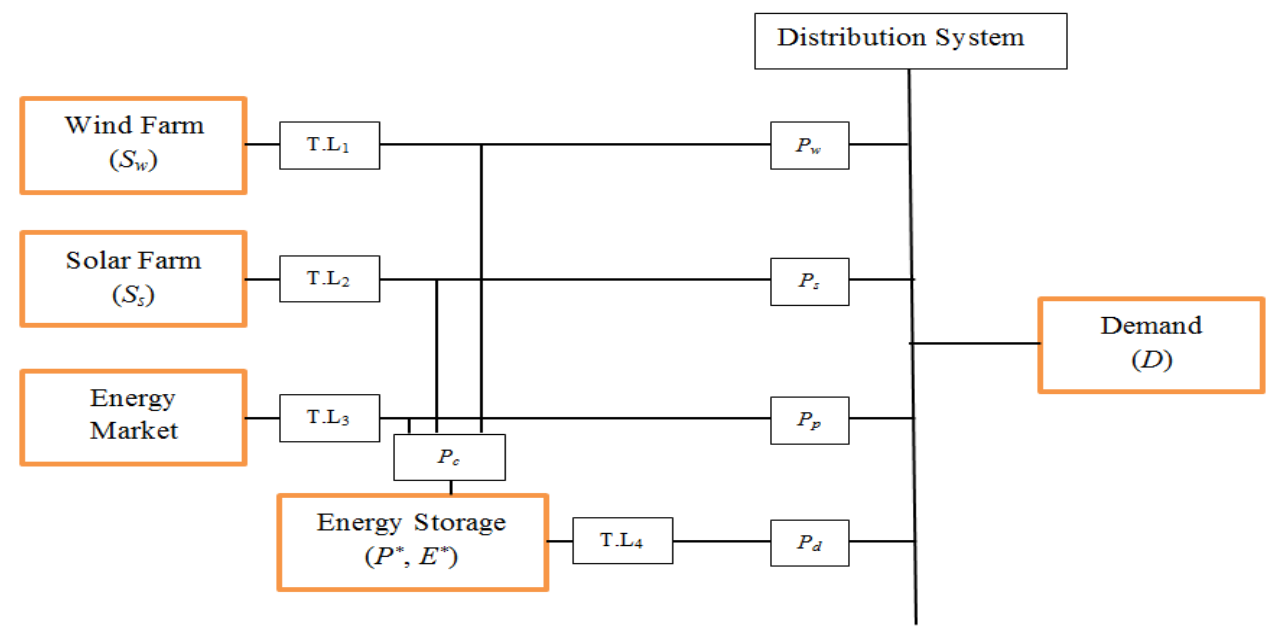

Figure 4.1 Proposed hybrid energy system connected to distribution system. 
The uncertainties in wind speed, solar radiation, energy market price, and load are modeled using Weibull distribution, Beta distribution, Lognormal distribution, and Normal distribution respectively. The time correlation between the time varying random variables of the proposed system has been reflected by using the proper random variable distribution for each time step in each season. The random variable distribution is created from historical data of wind speed, solar radiation, energy market price, and load for each time step on each season. The system modeling details are further explained in the following discussion. In these discussions the whole year is divided into seasons and each season is represented by a typical day of 24 hours.

\subsubsection{Wind farm}

As mentioned in chapter III, the capital cost of a wind farm consists of turbine system cost and the balance of the station cost. However, annual cost is typically used to represent the cost of energy. Hence, the capital cost of the wind farm is levelized over its lifespan time. The annual operating and maintenance cost is the summation of three different factors which are land lease cost, maintenance cost, and replacement cost.

Electrical power generated from wind turbine $\left(P_{w}\right)$ depends on wind speed $\left(v_{w}\right)$. The relationship between $P_{w}$ and $v_{w}$ is given in Equation 4.1,

$$
P_{w}=\left\{\begin{array}{cc}
0 & v_{w} \leq v_{\text {in }} \\
\frac{1}{2} \rho v_{w}^{3} \pi \frac{d^{2}}{4 * 10^{6}} C_{\rho} & v_{i n} \leq v_{w} \leq v_{r} \\
P_{r} & v_{r} \leq v_{w} \leq v_{\text {off }} \\
0 & v_{w} \geq v_{\text {off }}
\end{array}\right\}
$$


Where $P_{w}$ is the power generated from wind turbine in MW, $\rho$ is air density in $\mathrm{Kg} / \mathrm{m}^{3}, v_{w}$ is wind speed in $\mathrm{m} / \mathrm{s}, d$ is wind turbine diameter in $\mathrm{m}, C_{\rho}$ is wind turbine efficiency, $v_{\text {in }}$ is cut-in speed for wind turbine in $\mathrm{m} / \mathrm{s}, v_{r}$ is rated speed for wind turbine in $\mathrm{m} / \mathrm{s}, v_{\text {off }}$ is cutoff speed for wind turbine in $\mathrm{m} / \mathrm{s}$, and $P_{r}$ is rated power for wind turbine in MW.

The Weibull distribution is widely used for modeling the stochastic behavior of wind speed. The using of Weibull pdf to represent the stochastic nature of wind speed is based on a comparison of actual wind speed profiles at different sites and wind speed profiles estimated using the Weibull pdf [61]. The Weibull pdf is found to be valid for most wind speed profiles. Therefore, it has been used in this study to model the wind speed uncertainty over each time step on each season. The Weibull distribution pdf is given in Equation 4.2,

$$
f\left(v_{w}\right)=\left(\frac{k_{i . j}}{c_{i, j}}\right)\left(\frac{v_{w}}{c_{i, j}}\right)^{k_{i, j}-1} e^{-\left(\frac{v_{w}}{c_{i, j}}\right)^{k_{i, j}}}
$$

Where $c_{i, j}$ is Weibull scale parameter for season $i$ and time step $j$, and $k_{i, j}$ is Weibull shape parameter for season $i$ and time step $j$.

Wind speed data are usually available in time series, in which each data point represents either an instantaneous sample of wind speed or an average wind speed over some time period. The estimation of Weibull parameters over each time step is done using the empirical method which is a special case of the moment method [62]. The Weibull pdf parameters $k_{i, j}$ and $c_{i, j}$ are calculated using Equations 4.3 and 4.4, 


$$
\begin{gathered}
k_{i, j}=\left(\frac{\sigma_{w i, j}}{\mu_{w i, j}}\right)^{-1.086} \\
c_{i, j}=\frac{\mu_{w i, j}}{\Gamma\left(1+\frac{1}{k_{i, j}}\right)}
\end{gathered}
$$

Where $\sigma_{w i, j}$ is the standard deviation of wind speed data of time step $i$ and season $j, \mu_{w i, j}$ is the mean of wind speed data of time step $i$ and season $j$, and $\boldsymbol{\Gamma}$ is the gamma function.

The rating of utility scale wind farm is much larger than turbine rated power. Therefore, wind farm size is assumed to be a continuous function of wind speed. This is a reasonable assumption given that the wind farm is much bigger than a single turbine rating. This assumption is helpful in solving optimization problem. Now, the total power generated from wind farm in MW (wind farm utilization) can be calculated using Equations 4.5 and 4.6,

$$
\begin{gathered}
P_{w i, j}=C_{w i, j} * S_{w} \\
C_{w i, j}=\left\{\begin{array}{cc}
0 & v_{w i, j} \leq v_{i n} \\
\left(\frac{v_{w i, j}}{v_{r}}\right)^{3} & v_{i n} \leq v_{w i, j} \leq v_{r} \\
1 & v_{r} \leq v_{w i, j} \leq v_{o f f} \\
0 & v_{w i, j} \geq v_{o f f}
\end{array}\right\}
\end{gathered}
$$

Where $C_{w i, j}$ is wind farm utilization factor in season $i$ and time step $j$, and $S_{w}$ is wind farm size in MW. 


\subsubsection{Solar farm}

PV system cost has gone through rapid changes recently. The evolution of PV panel technology drives the price of PV systems to drop lower. The averaged capital cost of PV projects for utility scale solar farm in the US is $1820 \$ / \mathrm{KW}$, according to the most recent report from the Department of Energy in the United States [50]. While, the total operation and maintenance cost is $12 \$ / \mathrm{KW} / \mathrm{year}$. Similar to wind farm, the capital cost of solar farm will be levelized over its lifespan time and added to the total operation and maintenance cost to get the annual cost of energy.

The electrical power generated from $\mathrm{PV}$ panel $\left(P_{s}\right)$ is a function of solar radiation $s$. The relationship between $P_{s}$ and $s$ can be calculated using Equation 4.7 [51],

$$
P_{s}=s * A_{P V} * \eta_{P V}
$$

Where $P_{s}$ is electrical power generated from $\mathrm{PV}$ panel in $\mathrm{W}, s$ is global horizontal irradiance in $\mathrm{W} / \mathrm{m}^{2}, A_{P V}$ is $\mathrm{PV}$ panel area in $\mathrm{m}^{2}$, and $\eta_{P V}$ is efficiency of $\mathrm{PV}$ module.

Beta distribution is usually used to model solar radiation uncertainty. In this chapter, the Beta distribution for each time step in each season is used to model the uncertainty in solar radiation. The pdf of the Beta distribution is given in Equation 4.8,

$$
f_{b}(s)=\left\{\begin{array}{cc}
\frac{\Gamma\left(\alpha_{i, j}+\beta_{i, j}\right) s^{\alpha_{i, j}-1}(1-s)^{\beta_{i, j}-1}}{\Gamma\left(\alpha_{i, j}\right) \Gamma\left(\beta_{i, j}\right)} & 0 \leq s \leq 1 \\
0 & \text { Otherwise }
\end{array}\right\}
$$


Where $f_{b}(s)$ is Beta distribution function of $s$, and $\alpha_{i, j}, \beta_{i, j}$ are the parameters of Beta distribution for season $i$ and time step $j$.

The parameters of the Beta distribution for each time step is calculated form the historical solar radiation data using Equations 4.9 and 4.10,

$$
\begin{gathered}
\beta_{i, j}=\left(1-\mu_{s i, j}\left(\frac{\mu_{s i, j}\left(1+\mu_{s i, j}\right)}{\sigma_{s i, j}^{2}}-1\right)\right. \\
\alpha_{i, j}=\frac{\mu_{s i, j} \beta_{i, j}}{1-\mu_{s i, j}}
\end{gathered}
$$

Where $\mu_{s i, j}$ is the mean of solar radiation data for season $i$ and time step $j$, and $\sigma_{s i, j}$ is the standard deviation of the solar radiation data for season $i$ and time step $j$.

Now, the total solar farm power output (i.e. solar farm utilization) can be calculated using Equations 4.11 and 4.12,

$$
\begin{gathered}
P_{s i, j}=C_{s i, j} * S_{w} \\
C_{s i, j}=\frac{s_{i, j}}{s(S T C)}
\end{gathered}
$$

Where $C_{s i, j}$ is solar farm utilization factor for season $i$ and time step $j, S_{s}$ is solar farm size in MW, and $s(S T C)$ is global horizontal irradiance at slandered test condition which is $1000 \mathrm{~W} / \mathrm{m}^{2}$. 


\subsubsection{Energy storage}

In the suggested model, Lead acid batteries have been deployed as energy storage. Lead acid batteries are one of the oldest and most commercially mature form of rechargeable battery technology. Lead acid battery technologies are being used in wide range of applications like automotive, uninterruptible power supplies, and marine just to name a few. In Tappi Wind Park installed in 2001 by Hitachi, advanced lead acid batteries have been integrated with wind generating farms [5]. Energy storage in sizes of 10 to $20 \mathrm{MW}$ has been achieved using lead acid carbon technology [6].

Lead acid battery life cycle cost analysis is applied in this study. The cost estimates are based on capital cost, operation and maintenance cost, and battery replacement cost. The handbook from Sandia Labs describes different Lead Acid energy storage systems [5]. The average present value of installed energy storage system cost is $4000 \$ / \mathrm{KW}$ for power capacity and $400 \$ / \mathrm{MWh}$ for energy capacity of the energy storage. Therefore, the total cost of energy storage will be a function of its power and energy capacities.

In the proposed hybrid energy system, energy storage has two main goals. The first one is to smooth the fluctuation of power generated from the renewables. The second one is to reduce the total system cost by storing energy when it is cheap and supplying the stored energy when energy price and the demand are high. However, the power and energy capacities of the energy storage must be satisfied as shown in Equations 4.13 and 4.14, 


$$
\begin{gathered}
0 \leq P_{c i, j} \leq P^{*} \\
0 \leq P_{d i, j} \leq P^{*} \\
e_{i, j}=\left\{\begin{array}{cc}
e_{i, j-\Delta t}+\eta_{c} P_{c i, j} \Delta t & P_{c i, j} \geq 0 \\
e_{i, j-\Delta t}-\frac{1}{\eta_{d}} P_{d i, j} \Delta t & P_{d i, j} \geq 0 \\
0 \leq e_{i, j} \leq E^{*} &
\end{array}\right\}
\end{gathered}
$$

Where $P^{*}$ and $E^{*}$ are power and energy capacities of energy storage, $P_{c i, j}$ and $P_{d i, j}$ are power charged/discharged to/from energy storage, $\eta_{c}$ and $\eta_{d}$ are charging/discharging efficiencies of energy storage, $\Delta t$ is time step, and $e_{i, j}$ is energy stored in energy storage in season $i$ and time step $j$.

\subsubsection{Energy market}

Market operation determines the price of electricity purchased from the energy market. Therefore, the energy price is highly related with the demand level. The energy market, if available, will supply load requirements in the case that power generated from renewable sources is not enough or energy price is lower than LCOE from renewables. A model for the uncertainty in the market-clearing prices is needed. For pool-based electric energy market, the uncertainty of energy price is modeled using lognormal distribution [63]. The lognormal distribution is defined with two parameters $\mu_{\pi}$ and $\sigma_{\pi}$ that are, respectively, the mean and standard deviation of the variable's natural logarithm. The Lognormal distribution for each time step in each season is used to model the uncertainty in energy market price. The pdf of Lognormal distribution is given in Equation 4.15, 


$$
f(\pi)=\frac{1}{\sigma_{\pi i, j} \sqrt{2 \pi}} e^{-\frac{\left(\ln \pi-\mu_{\pi, j}\right)^{2}}{2 \sigma_{\pi i, j}^{2}}}
$$

Where $\pi$ is energy price in $\$ / \mathrm{MWh}, \mu_{\pi i, j}$ is the Log mean for season $i$ and time step $j$, and $\sigma_{\pi i, j}$ is the Log standard deviation for season $i$ and time step $j$.

Maximum likelihood procedure is used in estimating the lognormal distribution parameters as in Equations 4.16 and 4.17,

$$
\begin{gathered}
\mu_{\pi i, j}=\frac{\sum_{k} \ln \pi_{k i, j}}{n} \\
\sigma_{\pi i, j}^{2}=\frac{\sum_{k}\left(\ln \pi_{k i, j}-\mu_{\pi i, j}\right)^{2}}{n}
\end{gathered}
$$

Where $n$ is the number of data samples.

The total energy price purchased from energy market can be calculated using Equation 4.18,

$$
\sum_{i} \sum_{j} P_{p i, j} \pi_{i, j} \Delta t
$$

Where $P_{p i, j}$ is power purchased from energy market in season $i$ and time step $j$ in MW, $\pi_{i, j}$ is energy market price in season $i$ and time step $j$ in $\$ / \mathrm{MWh}$ and $\Delta t$ is time step in hours. 


\subsubsection{Load model}

The daily load variation over the long term is modeled using Normal probability distribution [64]. Normal distribution is often used in modeling real valued random variables whose distributions are not known. The Normal distribution is defined by two parameters which are the mean value $\mu_{l}$ and the standard deviation $\sigma_{l}$. Distribution system load data is used to create the normal distribution for each time step on each season in order to model the uncertainty in load demand. Then, the load profile for each time step will be created by calculating the two parameters which characterize the normal distribution over each time step. The pdf of the normal distribution is given in the Equation 4.19,

$$
f(l)=\frac{1}{\sigma_{l i, j} \sqrt{2 \pi}} e^{-\frac{\left(l-\mu_{i, j}\right)^{2}}{2 \sigma_{l i, j}^{2}}}
$$

Where $l$ is the load demand in MW, $\mu_{l i, j}$ is the mean value of $l$ for season $i$ and time step $j$, and $\sigma_{l i, j}$ is the standard deviation. The load will be supplied from the hybrid energy system and the energy market. 


\subsection{Problem formulation}

In this section, a mathematical formulation of the proposed system is presented for optimization. The objective of the optimization procedure is to minimize the annual cost of the overall planned system while considering system uncertainties, satisfying load requirements, and include consideration of system reliability. The system uncertainties are wind speed, solar radiation, energy market price and load. The load is supplied by the renewable sources and energy market. The system reliability problem is a result from failures of wind turbines, PV panels, energy market, energy storage, and transmission lines. Solving the optimization gives the optimal size of system components needed to minimize the overall annual cost of the system and meet problem constraints.

\subsubsection{Objective function}

Objective function measures the total system annual cost in (\$/year). The total system annual cost includes the levelized capital cost and operational and maintenance cost. Cost of system comes from wind turbines, PV panels, energy storage, and energy purchased from the market. The levelized cost for each system component will be calculated using Equation 4.20 [52],

$$
\zeta=\frac{\eta(1+\eta)^{N}}{(1+\eta)^{N}-1} * \gamma
$$


Where $\zeta$ is the levelized capital cost in $\$ /$ year, $\eta$ is discount rate of the investment in terms of annual interest per year, $N$ is lifespan of system component in years, and $\gamma$ is capital cost in $\$$.

Now, the objective function that measures total system annual cost can be formulated as in Equation 4.21,

$$
\min J=\left(\zeta_{w}+\beta_{w}\right) S_{w}+\left(\zeta_{s}+\beta_{s}\right) S_{s}+\zeta_{e 1} P^{*}+\zeta_{e 2} E^{*}+\sum_{i=1}^{4} \sum_{j=1}^{n_{s}} P_{p i, j} \tilde{\pi}_{i, j} \Delta t
$$

Where $\zeta_{w}$ is levelized wind farm cost in $\$ / \mathrm{MW} /$ year, $\zeta_{s}$ is levelized solar farm cost in \$/MW/year, $S_{w}$ is wind farm size in $\mathrm{MW}, S_{s}$ is solar farm size in MW, $\beta_{w}$ is operational and maintenance wind farm cost in $\$ / \mathrm{MW} /$ year, $\beta_{s}$ is operational and maintenance solar farm cost in $\$ / \mathrm{MW} / \mathrm{year}, \zeta_{e l}$ is levelized cost and operational costs for energy storage power capacity in $\$ / \mathrm{MW} / \mathrm{year}, \zeta_{e 2}$ is levelized cost and operational costs for energy storage energy capacity in $\$ / \mathrm{MWh} /$ year, $P^{*}$ and $E^{*}$ are power and energy capacities of energy storage in MW and MWh respectively.

$$
\sum_{i=1}^{4} \sum_{j=1}^{n_{s}} P_{p i, j} \tilde{\pi}_{i, j} \Delta t \text { is total cost of energy purchased from energy market in } \$ / \text { year }
$$

and $\tilde{\pi}_{i, j}$ is the energy price generated randomly using Lognormal distribution at season $i$ and time step $j, n_{s}$ is total number of time steps in one day. 


\subsubsection{Problem constraints}

1) Power balance constraint is that power generated from the renewables and purchased from the market is sufficient to satisfy the load demand as in Equation 4.22,

$$
\tilde{P}_{w}+\tilde{P}_{s}+P_{p}+P_{d}-P_{c}=\tilde{D}
$$

Where $\tilde{P}_{w}$ is the power generated from wind farm in MW, using randomly generated wind speed from Weibull distribution, calculated using Equation 4.23 and 4.24,

$$
\begin{gathered}
\tilde{P}_{w}=\tilde{C}_{w} * S_{w} \\
\tilde{C}_{w}=\left[\begin{array}{ccc}
\tilde{c}_{w 1,1} & \cdots & \tilde{c}_{w 1, n_{s}} \\
\vdots & \ddots & \vdots \\
\tilde{c}_{w 4,1} & \cdots & \tilde{c}_{w 4, n_{s}}
\end{array}\right]
\end{gathered}
$$

$\tilde{c}_{w i, j}$ is random wind farm utilization factor in season $i$ and time step $j$.

$\tilde{P}_{s}$ is the power generated from solar farm in MW, using randomly generated solar radiation from Beta distribution, calculated using Equation 4.25 and 4.26,

$$
\tilde{P}_{s}=\tilde{C}_{s} * S_{s}
$$




$$
\tilde{C}_{s}=\left[\begin{array}{ccc}
\tilde{c}_{s 1,1} & \cdots & \tilde{c}_{s 1, n_{s}} \\
\vdots & \ddots & \vdots \\
\tilde{c}_{s 4,1} & \cdots & \tilde{c}_{s 4, n_{s}}
\end{array}\right]
$$

$\tilde{c}_{s i, j}$ is random solar farm utilization factor in season $i$ and time step $j$.

$P_{p}$ is power purchased from the energy market in MW. This amount of power can be controlled during system operation.

$$
P_{p}=\left[\begin{array}{ccc}
p_{p 1,1} & \cdots & p_{p 1, n_{s}} \\
\vdots & \ddots & \vdots \\
p_{p 4, n_{s}} & \cdots & p_{p 4, n_{s}}
\end{array}\right]
$$

$p_{p i, j}$ is the power purchased from energy market in season $i$ and time step $j$.

$P_{d}$ is power discharged from energy storage in MW. This power can be controlled during system operation.

$$
P_{d}=\left[\begin{array}{ccc}
p_{d 1,1} & \cdots & p_{d 1, n_{s}} \\
\vdots & \ddots & \vdots \\
p_{d 4,1} & \cdots & p_{d 4, n_{s}}
\end{array}\right]
$$

$p_{d i, j}$ is the power discharged from energy storage in season $i$ and time step $j$.

$P_{c}$ is power charged into energy storage in MW. This power can be controlled during system operation. 


$$
P_{c}=\left[\begin{array}{ccc}
p_{c 1,1} & \cdots & p_{c 1, n_{s}} \\
\vdots & \ddots & \vdots \\
p_{c 4,1} & \cdots & p_{c 4, n_{s}}
\end{array}\right]
$$

$p_{c i, j}$ is the power charged into energy storage in season $i$ and time step $j$.

$\tilde{D}$ is the load demand in MW generated randomly from normal distribution for each time step.

$$
\tilde{D}=\left[\begin{array}{ccc}
\tilde{d}_{1,1} & \cdots & \tilde{d}_{1, n_{s}} \\
\vdots & \ddots & \vdots \\
\tilde{d}_{4,1} & \cdots & \tilde{d}_{4, n_{s}}
\end{array}\right]
$$

$\tilde{d}_{i, j}$ is the load demand generated from Normal distribution in season $i$ and time step $j$.

2) System reliability constraint

The reliability of the proposed system measured using expected energy not served (EENS). The EENS is a result of failures in wind turbines, PV panels, energy market, energy storage, and transmission lines. The calculation of EENS is illustrated in section 4.6. In this chapter, the reliability constraint is given in Equation (4.31),

$$
E E N S \leq E E N S_{\max }
$$

Where $E E N S_{\text {max }}$ is the maximum energy not serve as a percentage of the load. 
3) Energy storage constraints

Energy storage has two capacities that limit its operation which are the power and energy capacities.

a) Power capacity constraint: power charged/discharged into/from energy storage is limited by its power capacity.

$$
\begin{aligned}
& 0 \leq p_{c i, j} \leq P^{*} \\
& 0 \leq p_{d i, j} \leq P^{*}
\end{aligned}
$$

b) Energy capacity constraint: energy stored into is limited by its energy capacity.

$$
e_{i, j}=\left\{\begin{array}{cc}
e_{i, j-\Delta t}+\eta_{c} p_{c i, j} \Delta t & p_{c i, j} \geq 0 \\
e_{i, j-\Delta t}-\frac{1}{\eta_{d}} p_{d i, j} \Delta t & p_{d i, j} \geq 0
\end{array}\right\}
$$

From this section, the optimization variables can be classified into three major sets,

1) Decision variables: $S_{w}, S_{s}, P^{*}, E^{*}$. Once theses decision variables are determined, these hard to be changed.

2) Operating decision variables: $P_{p}, P_{c}, P_{d}$ which can be controlled and changed during system operation. 
3) Random variables: $\tilde{C}_{w}, \tilde{C}_{s}, \tilde{D}, \tilde{\pi}$ which are randomly generated using their stochastic distributions for each time step on each season.

Solving the optimization problem gives the optimal $\left(S_{w}, S_{s}, P^{*}\right.$, and $\left.E^{*}\right)$ needed to plan the hybrid energy system. However, in practical design there is an area limitation for wind/solar farm which limits the installed capacity of both of them. Therefore it is worth to indicate that an upper limit to the design variables (i.e. $S_{w}, S_{s}, P^{*}, E^{*}$ ) can be added into the optimization constraints. 


\subsection{Solution using stochastic programming}

\subsubsection{Compact matrix form}

In this subsection, the two stage stochastic programming is presented in compact matrix form to solve the optimization problem. The basic idea of two-stage stochastic programming is that optimal decisions should be based on data available at the time the decisions are made and should not depend on future observations. Two-stage formulation is widely used in stochastic programming. All first stage decision variables including wind farm size, solar farm size, energy storage power capacity, and energy storage energy capacity are denoted using $x$. Once these variables are determined, it is not practical to change them since they are installed and cannot be changed with the uncertainties. The first stage decision variables called 'here and now' variables. The second stage decision variables are contained in the vector $y_{d}$. This second stage decision variables are the operating decision variables including power purchased from energy market, power charged into energy storage, and power discharged from the energy storage. Now, the two stage stochastic programming can be written using the following matrix form.

$$
\begin{gathered}
\min c^{T} x+\frac{1}{|S|} \sum_{s \in S} q^{T} y_{d} \\
\text { s.t } A x \leq b \\
C_{s} x+H_{s} y_{d} \leq v \\
U_{s} x+V_{s} y_{d}=d_{s} \\
x \geq 0, y_{d} \geq 0
\end{gathered}
$$


The objective function (4.21) is represented by the vector form used in (4.34), where all first terms related to first stage decision variables are contained in $c^{T} x$ part and the terms of second stage decision variables are represented in vector $y_{d}$. Constraint (4.35) is not applied in the proposed procedure since there is no limitation on the budget or the installed capacity of the system components. The inequality constraints of EENS in (4.31) and energy storage operation (4.32) and (4.33) are expressed in (4.36). Equality constraint, namely, energy balance constraint (4.22) is included in (4.37). The $C_{s}, H_{s}, U_{s}$, and $V_{s}$ matrices express the matrix in (4.24), (4.26), (4.27), (4.28), and (4.29). $d_{s}$ represent (4.30). $q^{T} y_{d}$ is the cost of the recourse action which is related to the price of energy purchased from the market. All decision variables are positive, as included in (4.38).

\subsubsection{Sample average approximation}

The expected operation cost can be approximated by means of sampling as shown in the second term of Equation (4.34). This term is a SAA of the expected operation cost. However, the solution of this sample based technique does not guarantee optimality in the original problem. As shown in [65], for sample size of $S$, consider $x_{S}{ }^{*}$ to be the optimal solution and $J_{S}{ }^{*}$ to be the optimal objective value of the approximated problem in (4.34). Since $x^{*}, J^{*}$ are the optimal solution of the original problem, then

$$
J^{*} \leq J_{S}^{*}
$$


Therefore, $J_{S}{ }^{*}$ represents an upper bound of optimal objective value. But, $J_{S}{ }^{*}$ is the optimal solution of (4.34), therefore the following holds true,

$$
J_{s}^{*}=J_{s}\left(x_{s}^{*}\right) \leq J_{s}\left(x^{*}\right)
$$

Taking expectation for both sides

$$
E\left[J_{s}^{*}\right] \leq E\left[J_{s}\left(x^{*}\right)\right]
$$

Since SAA is an unbiased estimator of the population mean

$$
E\left[J_{s}^{*}\right] \leq E\left[J_{s}\left(x^{*}\right)\right]=J^{*}
$$

Thus, $E\left[J_{s}^{*}\right]$ is a lower bound for the optimal objective value. Now, the lower and upper bound estimates are discussed. The derivation of lower and upper bound estimates is presented in [66].

1) Lower bound estimates

$E\left[J_{s}^{*}\right]$ can be estimated using $M_{L}$ independent batches, each of them contains $S_{L}$ samples. Solving SAA problem gives $J_{S_{L}}^{*}$ then lower bound for this $S_{L}, M_{L}$ is calculated as Equation (4.43) 


$$
L_{S_{L}, M_{L}}=\frac{1}{M_{L}} \sum_{s=1}^{M_{L}} J_{S_{L}}^{*}
$$

From the central limit theorem, the distribution of lower bound estimate converges to Normal distribution. The mean value of this Normal distribution is $E\left[J_{S_{L}}^{*}\right]$, which is approximated by a sample mean $L_{S_{L}}, M_{L}$ and $\operatorname{Var}\left[J_{S_{L}}^{*}\right]$ approximated by sample variance.

$$
N_{L}^{2}=\frac{1}{M_{L}-1} \sum_{s=1}^{M_{L}}\left(J_{S_{L}}^{*}-L_{S_{L}, M_{L}}\right)^{2}
$$

Now, for $\theta$ confidence interval, the two sided $100(1-\theta) \%$ lower bound is

$$
\left[L_{S_{L}, M_{L}}-\frac{s_{\theta / 2} N_{L}}{\sqrt{M_{L}}} \quad L_{S_{L}, M_{L}}+\frac{s_{\theta / 2} N_{L}}{\sqrt{M_{L}}}\right]
$$

Where $s_{\theta / 2}$ satisfies $\operatorname{Pr}\left\{-s_{\theta / 2} \leq N(0,1) \leq s_{\theta / 2}\right\}=1-\theta$.

The lower bound confidence interval computed solving $M_{L}$ independent SAA problem of sample size $N_{L}$. 


\section{2) Upper bound estimates}

Given $x_{S}{ }^{*}$, the upper bound of the optimal objective value can be calculated using $M_{U}$ independent batches with $S_{U}$ samples in each batch. Since the solution is $x_{S}{ }^{*}$, the problem can be decomposed to $S_{U}$ independent linear programming, where each one gives $J_{S_{U}}^{s}\left(x_{s}^{*}\right)$. Then the upper bound is approximated from Equation 4.46

$$
U_{S_{U}, M_{U}}=\frac{1}{M_{U}} \sum_{s=1}^{M_{U}} J_{S_{U}}^{s}\left(x_{s}^{*}\right)
$$

Using central limit theorem, the distribution of an upper bound estimate converges to a

normal distribution with mean $E\left[J_{S_{u}}\left(x_{s}^{*}\right)\right]$ which is approximated by a sample mean $U_{S_{U}, M_{U}}$ and variance $\operatorname{Var}\left[J_{S_{u}}\left(x_{s}^{*}\right)\right]$ which can be approximated by a sample variance

$$
N_{U}^{2}\left(x_{N}^{*}\right)=\frac{1}{S_{U}-1} \sum_{s=1}^{M_{u}}\left(J_{S_{U}}^{s}\left(x_{s}^{*}\right)-U_{S_{U}}, M_{U}\left(x_{s}^{*}\right)\right)^{2}
$$

The two sided confidence interval of the upper bound is calculated same as for lower bound.

A solution $x_{S}{ }^{*}$ is found from each batch of $M_{L}$ in lower bound SAA problems and used to find upper bound estimates. 


\subsection{Scenario aggregation procedure}

In this, a simpler approach is explored to find the optimal near solution. Using the probability distribution, a scenario of wind, solar, market and load is created for the whole year using the typical days. Then for this scenario, using LP described in section 4.3, an optimal solution is found for the decision variables as shown in the flow diagram in Figure 4.2. Now this solution is valid for the given values of the sampled scenario.

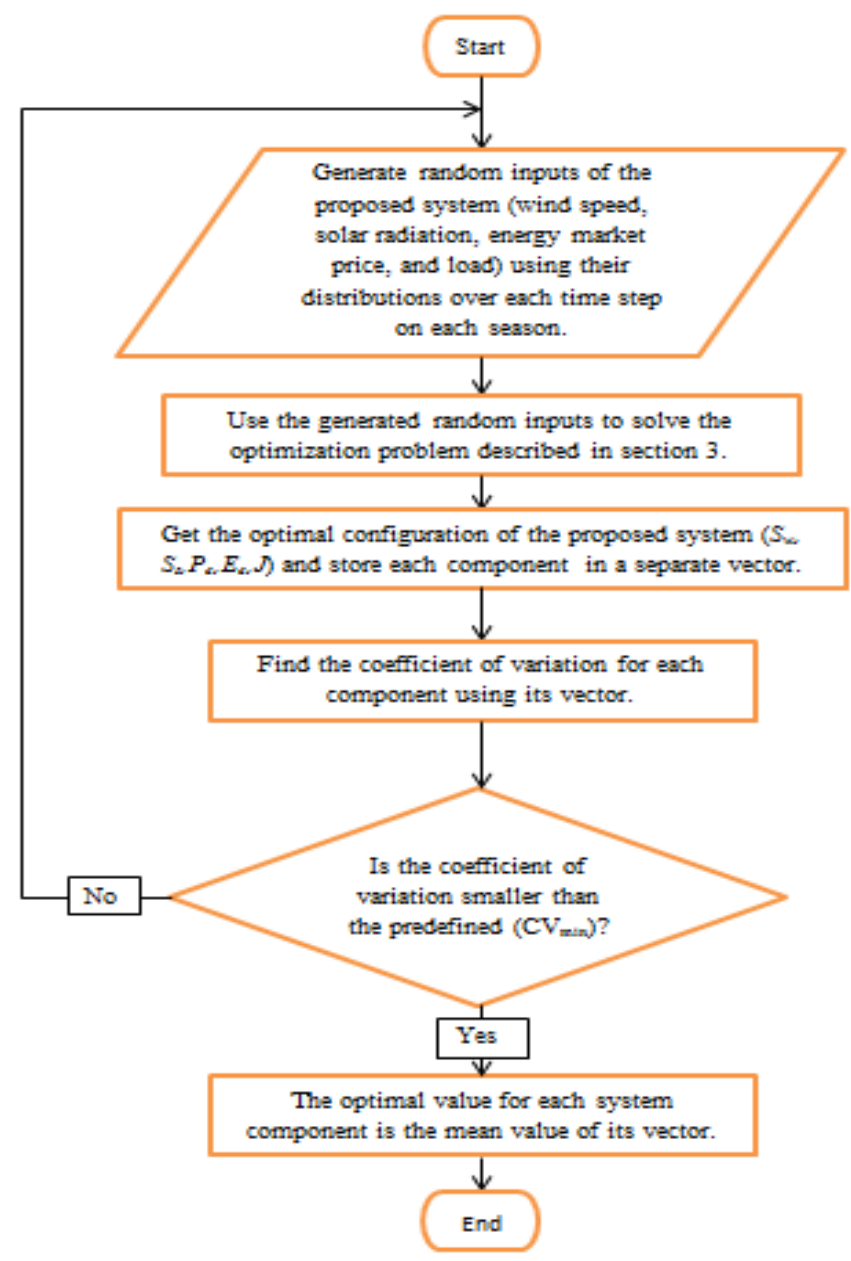

Figure 4.2 Scenario aggregation flow chart. 
To decide on the near optimal solution, we do clustering to see how the solutions group together. The clustering technique used is K-means, with K-means++ procedure proposed in [67] used to determine clusters centers. The K-means++ procedure improves the simulation time of Lloyd's algorithm, and the quality of final solution. The clustering procedure is illustrated in the following discussion.

\subsubsection{Cluster centers}

Let $D(x)$ be the shortest distance between the closest center and observation $x$. The cluster seeds are obtained following these steps.

1) An observation is selected uniformly at random from data set $X$ and this observation will be the first center for the cluster number $1 c_{l}$.

2) Choose the next center $c_{i}$, selecting $c_{i}=x^{\prime} \in X$ with probability $\frac{D\left(x^{\prime}\right)^{2}}{\sum_{x \in X} D(x)^{2}}$.

3) Repeat step 2 until $k$ cluster centers are chosen.

\subsubsection{K-means algorithm}

1) Choose $k$ initial centers as mentioned earlier $C=\left(c_{1}, \ldots, c_{k}\right)$.

2) The cluster $C_{i}$ contains observations of $X$ that are closer to $c_{i}$ than they are to $c_{j}$ for all $j \neq i$.

3) The center of mass of all points is the average of the cluster $C_{i}$ observations. 


\subsection{System reliability modeling}

The reliability of the proposed system will be measured using expected value of energy not served to the load (EENS). The energy not served in the proposed system results from the failure of system components which are wind turbines, PV panels, energy market, energy storage and transmission lines. Then, the EENS will be added to the constraints and applied to the optimization problem, such that, EENS is less than

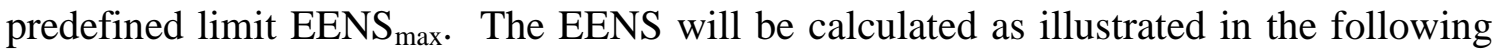
discussion.

\subsubsection{Expected energy not served from wind farm}

The EENS from wind farm in the proposed system is a result from two scenarios. The first one is the failure of wind turbines which can cause a partial loss of wind farm utilization. The second scenario is transmission line failure which results in a total loss of wind farm utilization. The outage probability of wind turbine is found to be related to wind speed [68]. The relationship between wind speed and wind turbine outage probability is shown in Equation 4.48,

$$
p_{W . T d}=a v_{w}^{3}+b v_{w}^{2}+c v_{w}+d
$$

Where $p_{W . T d}$ is the probability of wind turbine to be down. a, b, c, and d coefficients are taken from [69] and given in Table 4.1. 
Table 4.1: Coefficients of wind turbine failure probability

\begin{tabular}{cccc}
\hline $\mathrm{a}$ & $\mathrm{b}$ & $\mathrm{c}$ & $\mathrm{d}$ \\
\hline$-4.4655^{*} 10^{-5}$ & $2.9438 * 10^{-3}$ & -0.04538 & 0.2253 \\
\hline
\end{tabular}

For transmission line, the outage probability is calculated using Equation 4.49,

$$
p_{T . L d}=\frac{\lambda_{T . L}}{\lambda_{T . L}+\mu_{T . L}}
$$

Where $\lambda_{T . L}$ is the failure rate of transmission line, and $\mu_{T . L}$ is the repair rate of transmission line.

Now, the probability of $k$ wind turbines to be down is calculated using Equation 4.50

$$
k\left(\begin{array}{c}
N_{w} \\
k
\end{array}\right)\left(p_{W . T d}\right)^{k}\left(1-p_{W . T d}\right)^{N_{w}-k}
$$

Where $k$ is number of down wind turbines, and $N_{w}$ is total number of wind turbines. Then, EENS from wind farm will be the combination of the two scenarios. 


\subsubsection{Expected energy not served from solar farm}

The EENS from solar farm in the proposed system is the same as wind farm a result from two scenarios. The first one is the failure of PV panels which can cause a partial loss of solar farm utilization. While, the second scenario is transmission line failure which results in a total loss of solar farm utilization. The outage probability of PV panel can be calculated using Equation 4.51,

$$
p_{P V d}=\frac{\lambda_{P . V}}{\lambda_{P . V}+\mu_{P . V}}
$$

For transmission line, the outage probability is the same one used in wind turbine.

Now, the probability of $k \mathrm{PV}$ panels to be down is calculated using Equation 4.52 ,

$$
k\left(\begin{array}{c}
N_{s} \\
k
\end{array}\right)\left(p_{P V d}\right)^{k}\left(1-p_{P V d}\right)^{N_{s}-k}
$$

Where $k$ is number of down PV panels, $N_{s}$ is total number of PV panels. Then, EENS from solar farm will be the combination of the two scenarios.

\subsubsection{Expected energy not served from energy market}

The energy market in the proposed system is needed to purchase the energy in order to meet load demand when the renewables are not sufficient or when the energy 
market price is lower than renewables LCOE. The reliability model of the energy market suggested is either the market to be up or down. Thus, there is no partial outage of energy purchased from the market. The energy market is supposed to have failure rate of $\lambda_{m}$ and repair rate of $\mu_{m}$. Hence, the probability of energy market to be down is given in Equation 4.53,

$$
p_{m \cdot d}=\frac{\lambda_{m}}{\lambda_{m}+\mu_{m}}
$$

Now, to calculate the EENS from the market the combination of energy market failure and transmission line failure are combined together.

\subsubsection{Expected energy not served from energy storage}

The energy storage EENS calculation procedure will be the same as the energy market.

Finally, the total EENS will be the summation of the four cases mentioned in this section. 


\subsection{Case study and results}

In this case study, a grid connected distribution system in north Texas is used for illustrating the methodology developed. The uncertainties in wind speed, solar radiation, energy market price, and load are modeled using the stochastic distribution models mentioned earlier. The Weibull, Beta, Lognormal and normal probability distribution functions that are needed to estimate the wind speed, solar irradiance, energy market price, and load demand are built based on one year of historical data that have been collected from north Texas. The year is divided into four seasons, with each season being represented by one typical day. Then, the day representing each season is subdivided into 48 time steps. Considering a month to be 30 days, each time step has around 90 data points in one year. The mean and standard deviation for each time step are calculated using these data points. Finally, the Weibull, Beta, Lognormal and Normal distributions are created for each time segment on each day that represents a particular season as described in section 4.2. By using this procedure, the time correlation between these variables will be included.

The wind farm uses the National Renewable Energy Laboratory (NREL) wind turbine design cost and scaling model which has the specifications shown in Table 4.2 [55], and is assumed to have a lifespan of 25 years. The wind speed data has been collected from the NREL for the northern area distribution system. The available wind speed data is averaged over 30 minute time intervals. However, the wind speed is measured using anemometer at $50 \mathrm{~m}$ height, so Equation 4.54 has been used to substitute the impact of the roughness of the earth's surface on wind speed, 


$$
\left(\frac{v_{w}}{v_{o}}\right)=\left(\frac{H}{H_{o}}\right)^{\sigma}
$$

Where $\sigma$ is the friction coefficient, $v_{w}$ is wind speed at hub height $(82.5 \mathrm{~m}) \mathrm{in} \mathrm{m} / \mathrm{s}, v_{o}$ is wind speed at anemometer height $(50 \mathrm{~m})$ in $\mathrm{m} / \mathrm{s}, H$ is turbine hub height $(82.5 \mathrm{~m})$, and $H_{o}$ anemometer height $(50 \mathrm{~m})$.

The wind farm cost model is developed based on the 2014 wind technologies market report disseminated by the U.S. Department of Energy (DOE) [54]. The average capital cost for utility scale wind farm projects is $1550 \$ / \mathrm{KW}$, and the annual operating and maintenance cost is $15 \$ / \mathrm{KW} /$ year.

The wind farm utilization factor $\left(c_{w}\right)$ for four different typical days in different seasons, as an example, is shown in Figure 4.3. One can note that wind farm electrical power production is related to time. The wind power during the day time is most likely less than the wind power during the night (i.e. wind speed during night is higher than wind speed during day time).

Table 4.2: Wind turbine characteristics.

\begin{tabular}{cc}
\hline Turbine rated power $(\mathrm{MW})$ & 1.91 \\
\hline Turbine rotor diameter $(\mathrm{m})$ & 96.7 \\
\hline Turbine hub height $(\mathrm{m})$ & 82.5 \\
\hline Rotor peak power coefficient $\left(C_{p}\right)$ & 0.47 \\
\hline Cut in wind speed $(\mathrm{m} / \mathrm{s})$ & 2.5 \\
\hline Cut off wind speed $(\mathrm{m} / \mathrm{s})$ & 25.0 \\
\hline Rated wind speed $(\mathrm{m} / \mathrm{s})$ & 9.6 \\
\hline
\end{tabular}




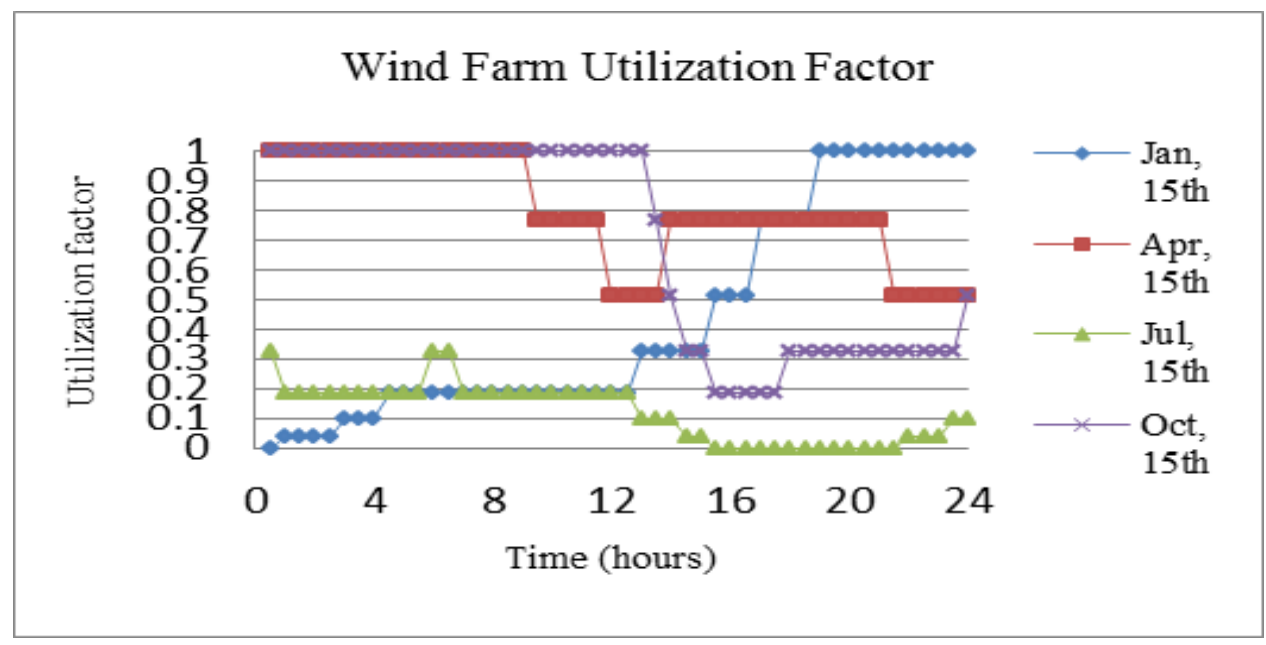

Figure 4.3 Wind farm utilization factor.

One year of global horizontal irradiance data averaged over 30 minute time intervals is obtained from NREL. The averaged capital cost of PV projects in the United States for utility scale is $1820 \$ / \mathrm{KW}$ and the total operation and maintenance cost is $12 \$ / \mathrm{KW} /$ year [50]. The lifespan of the solar farm in this work is assumed to be 33 years. The solar farm utilization factor $\left(c_{s}\right)$ for the same four days is shown in Figure 4.4. The reader can observe that power generated from solar farm is related to time. Solar power is generated during the day time only, since it depends on solar radiation. 


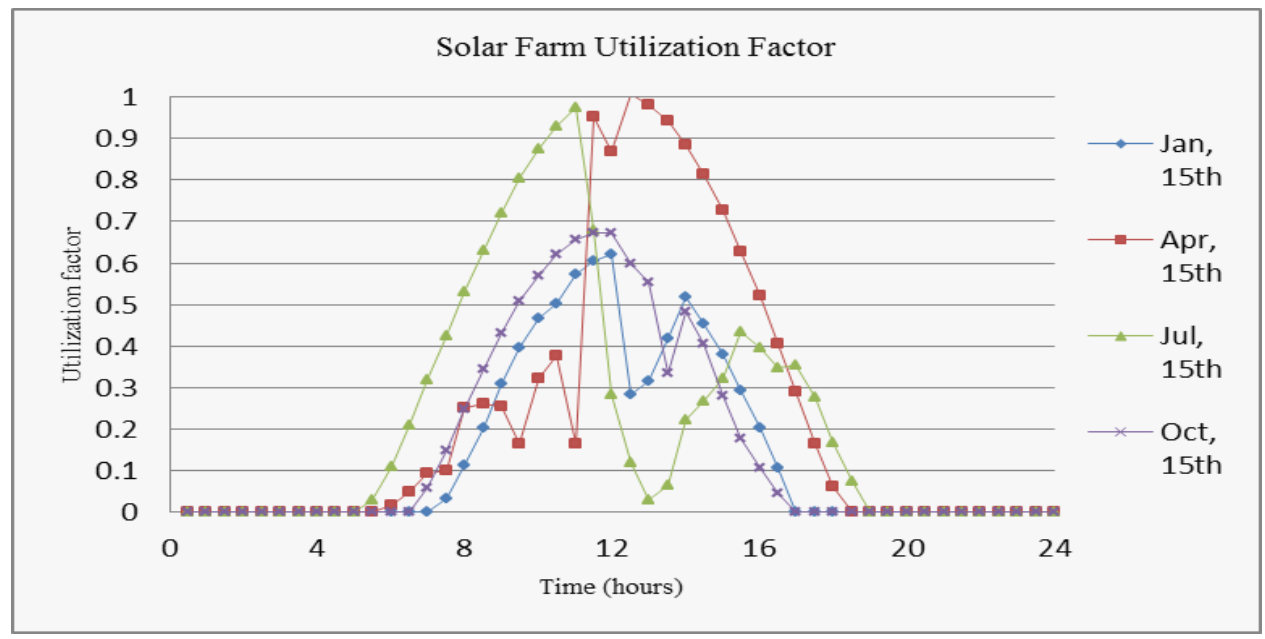

Figure 4.4 Solar farm utilization factor.

Energy market price is collected from Electric Reliability Council of Texas (ERCOT). One year of energy price data averaged over 30 minute time intervals is obtained. The energy price for four different days in four different seasons, as an example, is shown in Figure 4.5. The energy price during the day time is typically more than energy price during the night time since the demand is higher at the day time.

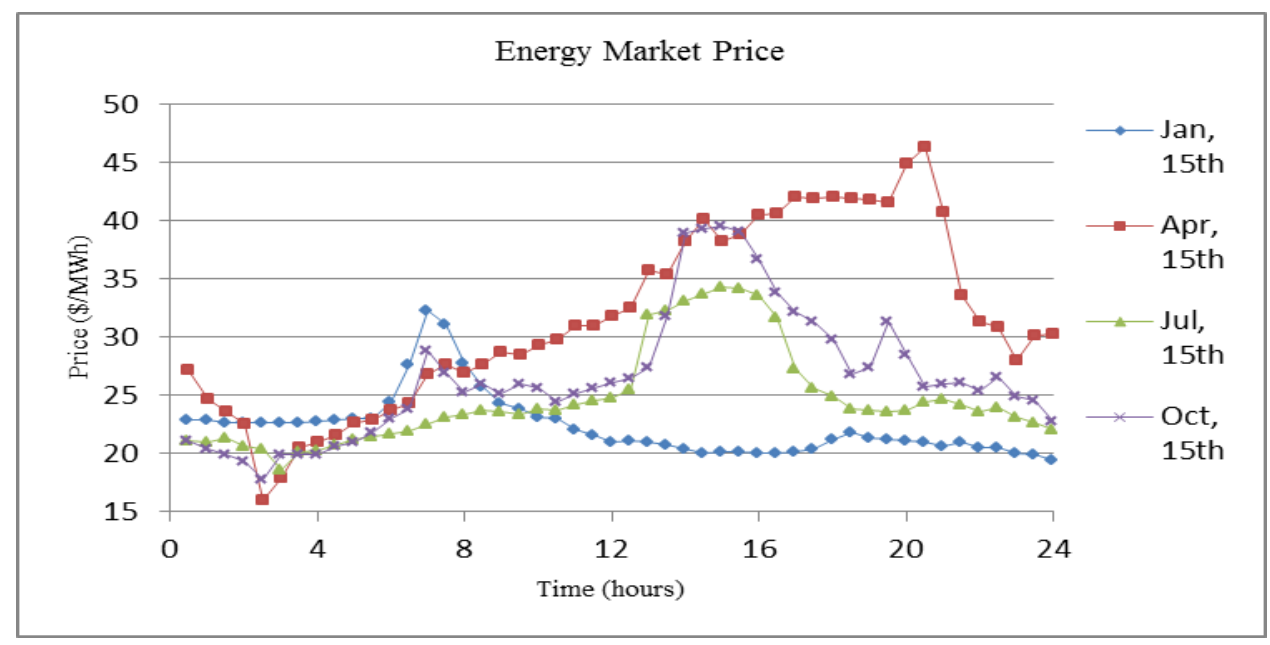

Figure 4.5 Energy price over the time. 
The load profile for north Texas distribution system is obtained from ERCOT. One year of load data averaged over 30 minute time steps is collected. The average load value is $925.25 \mathrm{MW}$ while the load peak value is $1689.9 \mathrm{MW}$. Four days load data over the time, for example, is shown in Figure 4.6. The load during the day time is usually more than the load during the night.

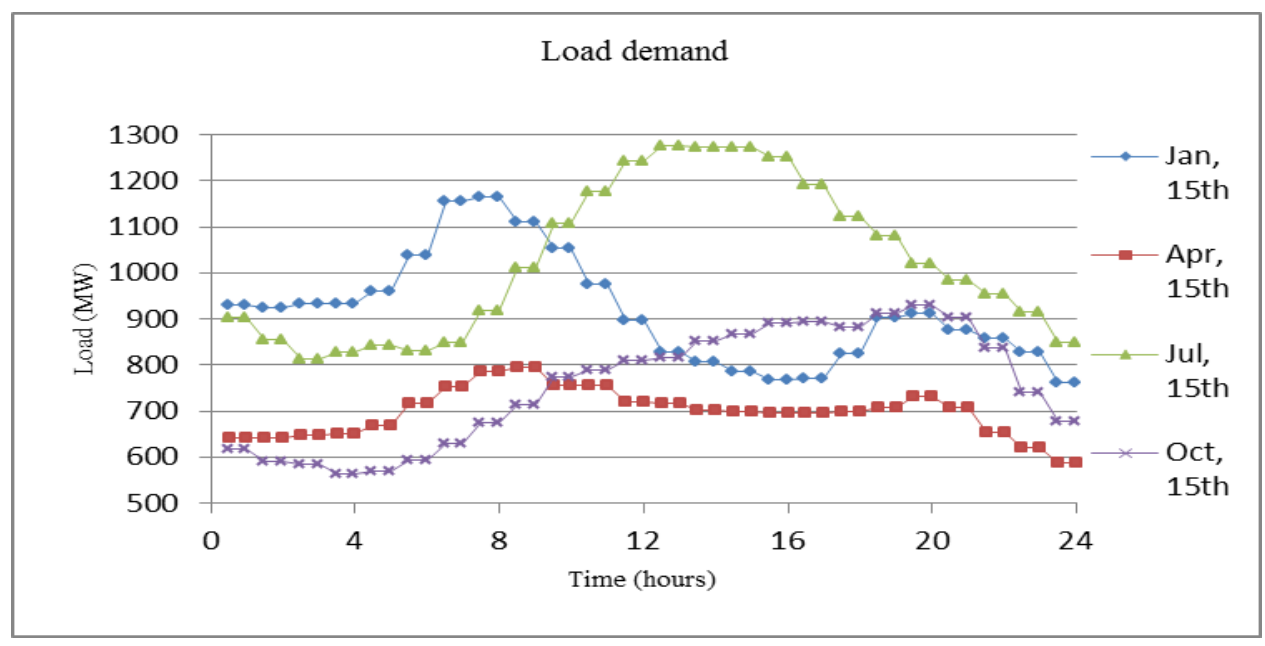

Figure 4.6 Averaged load over the time.

Two stage stochastic programming and scenario aggregation procedure are used to solve the optimization problem and the results are compared. Xprog MATLAB tool box is used to solve the two stage stochastic programming [56].

\subsubsection{Case one: fully reliable system}

In case one, the system is considered with the uncertainties in wind speed, solar radiation, energy market price, and load only. The reliability of the system is not 
included in the optimization procedure. One year worth's of wind speed, solar radiation, energy market price, and load data used to create the stochastic distributions over each time step in each season. The parameters of each random distribution for each time step on each season are given in Figures 4.7 through 4.14. The hybrid energy system planning result for this case is solved using two stage stochastic programming described earlier with different number of simulated scenarios and batches. Five different sample sizes have been chosen which are 500, 1000, 2500, 5000, and 10000. Lower bound estimate of each sample size is found by solving SAA with $M_{L}$ equal 5 . Then, these solutions are used to calculate upper bound estimate. The lower bound results for the objective function are given in Table 4.3 and the upper bound results are given in Table 4.4 .

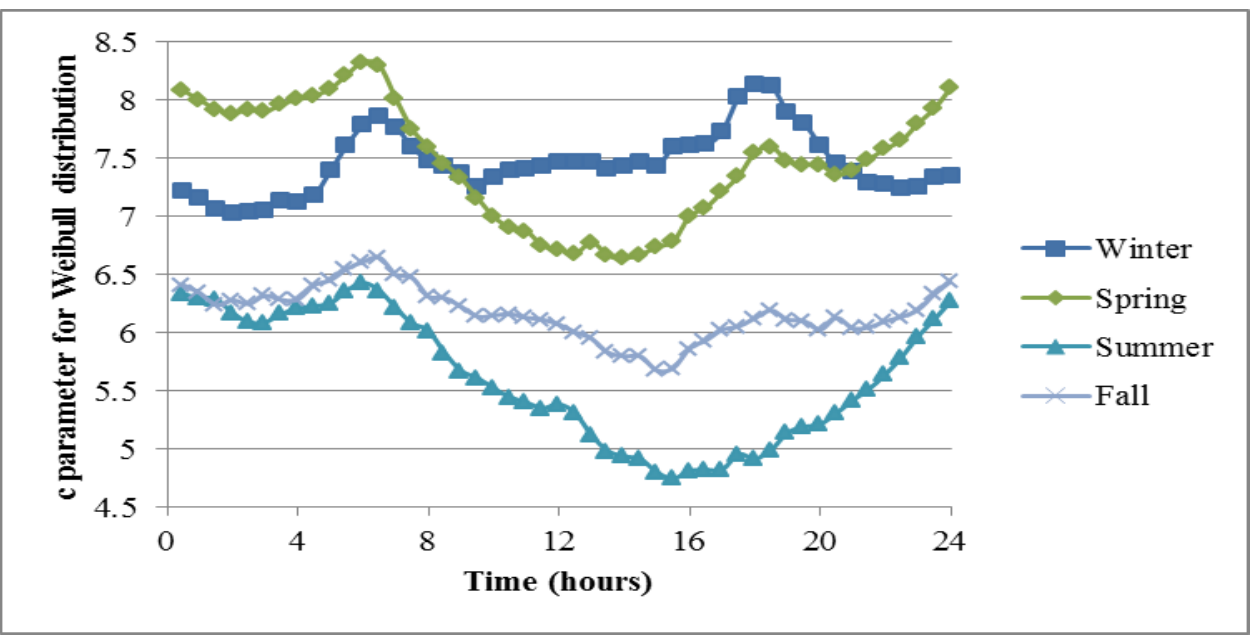

Figure 4.7 Scale parameter for Weibull distribution. 


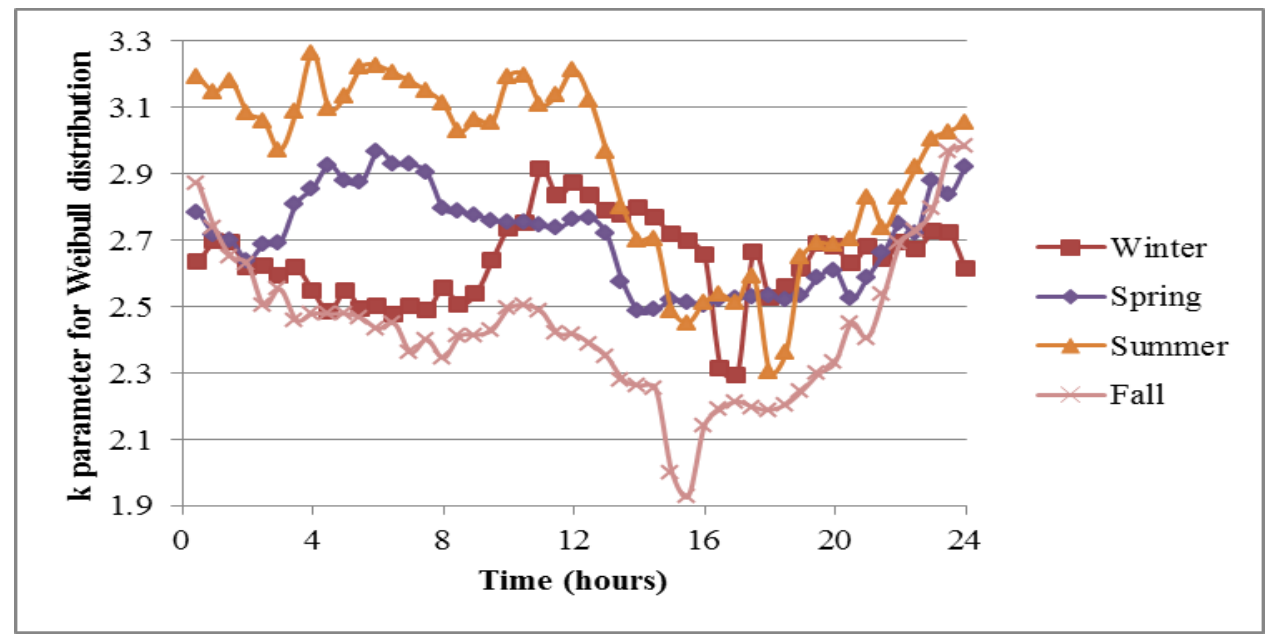

Figure 4.8 Shape parameter for Weibull distribution.

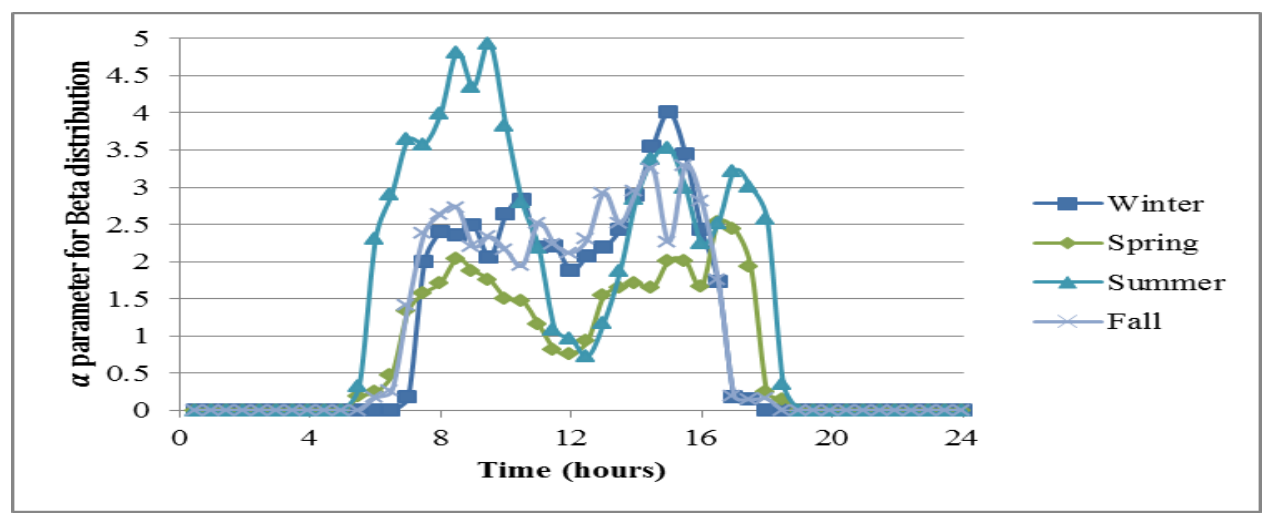

Figure $4.9 \alpha$ parameter for Beta distribution.

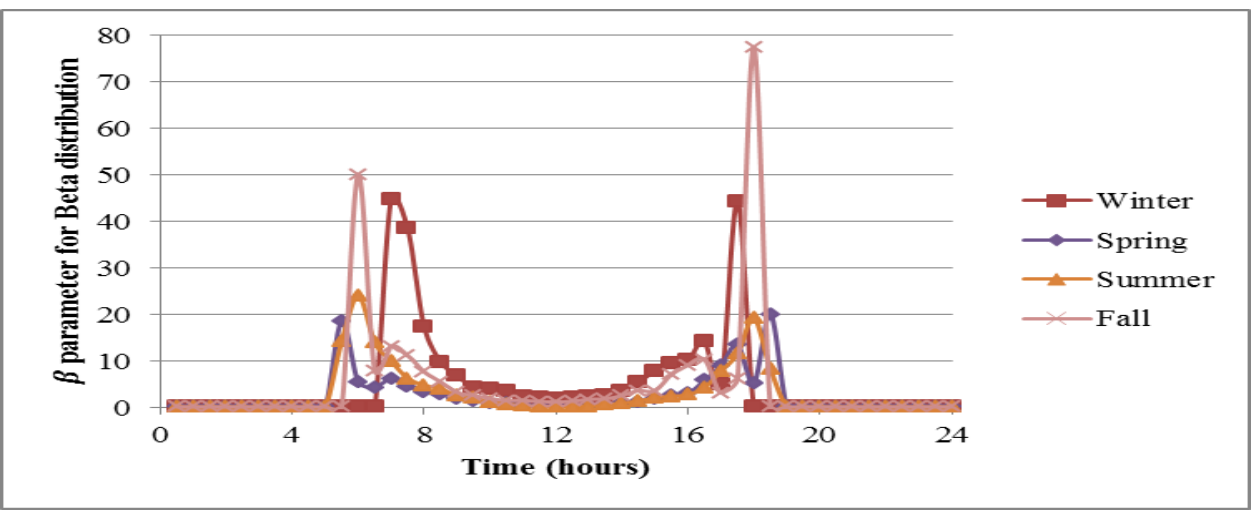

Figure $4.10 \beta$ parameter for Beta distribution. 


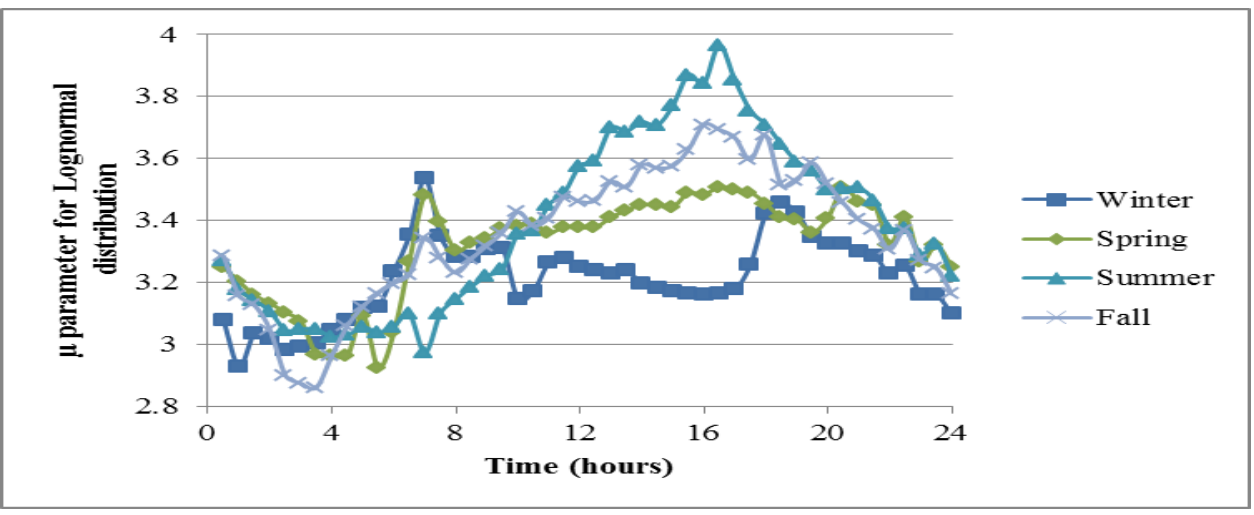

Figure 4.11 Log mean parameter for Lognormal distribution.

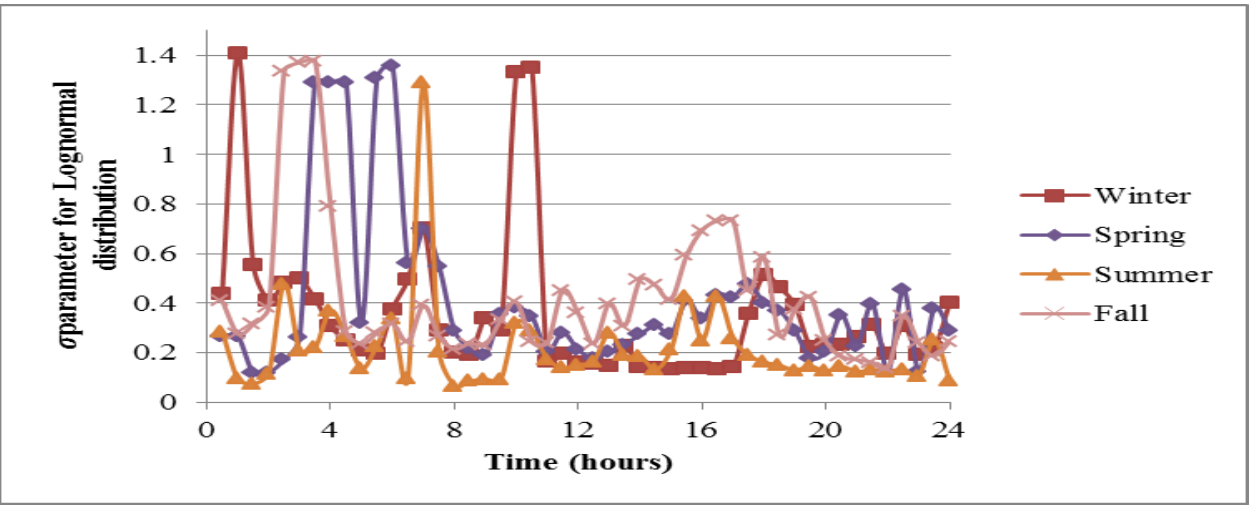

Figure 4.12 Log standard deviation parameter for Lognormal distribution.

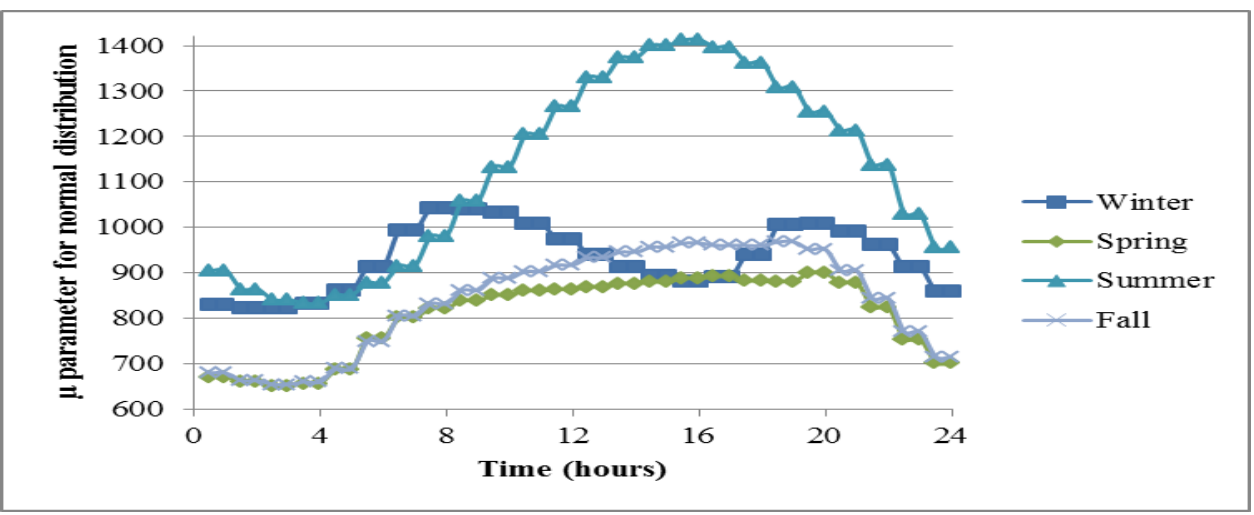

Figure 4.13 Mean parameter for Normal distribution. 


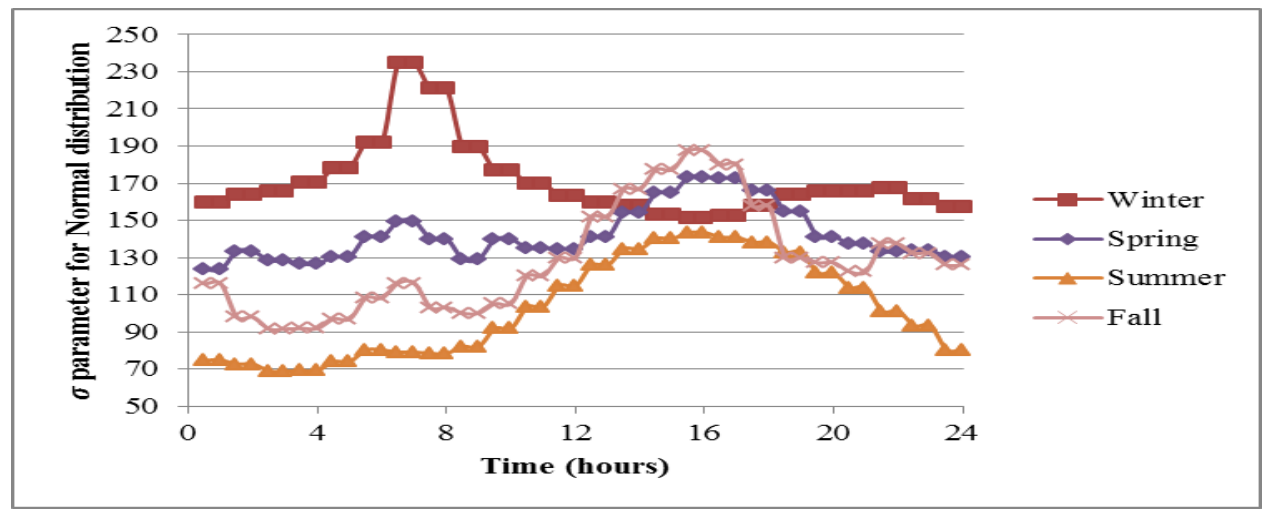

Figure 4.14 Standard deviation parameter for Normal distribution.

Table 4.3: Lower bound estimate from stochastic programming

\begin{tabular}{|c|c|}
\hline Sample size & Lower bound (\$m, 95\% confidence interval) \\
\hline 500 & $268.74 \pm 17.85$ \\
\hline 1000 & $279.51 \pm 16.54$ \\
\hline 2500 & $284.21 \pm 13.8$ \\
\hline 5000 & $286.3 \pm 3.2$ \\
\hline 10000 & $287.34 \pm 4.58$ \\
\hline
\end{tabular}


Table 4.4: Upper bound estimate from stochastic programming

\begin{tabular}{|c|c|c|c|c|c|c|}
\hline Sample size & Batch & $S_{w}(\mathrm{MW})$ & $S_{s}(\mathrm{MW})$ & $P^{*}(\mathrm{MW})$ & $E^{*}(\mathrm{MW})$ & Upper bound (\$m) \\
\hline \multirow[t]{5}{*}{500} & 1 & 448.4 & 53.8 & 23.1 & 156.6 & $271 \pm 3.7$ \\
\hline & 2 & 472.5 & 52.5 & 21.4 & 157.1 & $274.9 \pm 2.3$ \\
\hline & 3 & 476.6 & 46.0 & 22.0 & 171.6 & $272.6 \pm 6.3$ \\
\hline & 4 & 472.4 & 47.4 & 19.7 & 168.3 & $276.7 \pm 3.5$ \\
\hline & 5 & 462.5 & 46.7 & 21.2 & 143.7 & $275.8 \pm 1.3$ \\
\hline \multirow[t]{5}{*}{1000} & 1 & 471.9 & 43.1 & 24.8 & 144.8 & $285.7 \pm 8.9$ \\
\hline & 2 & 461.9 & 44.5 & 22.7 & 157.7 & $279.7 \pm 7.6$ \\
\hline & 3 & 479.8 & 47.1 & 22.6 & 140.6 & $280.8 \pm 4.3$ \\
\hline & 4 & 457.6 & 43.5 & 21.2 & 149.8 & $287.7 \pm 4.6$ \\
\hline & 5 & 477.5 & 52.7 & 22.4 & 143.4 & $284.7 \pm 3.8$ \\
\hline \multirow[t]{5}{*}{2500} & 1 & 461.4 & 42.9 & 23.1 & 159.6 & $284.3 \pm 6.4$ \\
\hline & 2 & 467.9 & 43.4 & 23.4 & 154.3 & $288.7 \pm 4.8$ \\
\hline & 3 & 476.9 & 42.6 & 22.0 & 150.0 & $290.4 \pm 4.0$ \\
\hline & 4 & 482.3 & 43.4 & 21.8 & 149.4 & $295.8 \pm 6.5$ \\
\hline & 5 & 457.8 & 46.5 & 24.9 & 143.7 & $287 \pm 3.6$ \\
\hline \multirow[t]{5}{*}{5000} & 1 & 468.9 & 46.8 & 22.6 & 151.3 & $296.4 \pm 4.1$ \\
\hline & 2 & 466.6 & 45.8 & 23.3 & 150.6 & $293.1 \pm 4.2$ \\
\hline & 3 & 462.3 & 46.8 & 23.1 & 152.9 & $290.2 \pm 5.5$ \\
\hline & 4 & 461.5 & 47.1 & 23.4 & 151.2 & $293.2 \pm 8.2$ \\
\hline & 5 & 461.7 & 45.7 & 23.4 & 152.0 & $290.1 \pm 3.5$ \\
\hline \multirow[t]{5}{*}{10000} & 1 & 459.5 & 45.4 & 23.6 & 150.3 & $294.3 \pm 8.5$ \\
\hline & 2 & 461.1 & 45.9 & 23.6 & 150.2 & $294.7 \pm 2.8$ \\
\hline & 3 & 461.1 & 46.0 & 22.9 & 152.1 & $297.2 \pm 1.5$ \\
\hline & 4 & 463.5 & 46.3 & 23.3 & 148.4 & $293.8 \pm 2.4$ \\
\hline & 5 & 463.4 & 46.5 & 22.7 & 148.7 & $289.4 \pm 3.5$ \\
\hline
\end{tabular}


Next, the optimal solution is chosen such that the Coefficient of Variation of each system component value, in all batches that have same number of samples is less than $2 \%$. Therefore the optimal configuration happens at sample size 5000 and 10000 . To compare the results, Scenario aggregation procedure described earlier has been used. The results for near optimal planning configuration of 1000 generated samples are given in Table 4.5 and the clusters are given in Table 4.6.

Table 4.5: Suboptimal planning result from scenario aggregation

\begin{tabular}{lc}
\hline Wind farm size $\left(S_{w}\right)$ & $473.81 \mathrm{MW}$ \\
\hline Solar farm size $\left(S_{s}\right)$ & $33.88 \mathrm{MW}$ \\
\hline Storage power capacity $\left(P^{*}\right)$ & $15.46 \mathrm{MW}$ \\
\hline Storage energy capacity $\left(E^{*}\right)$ & $131.76 \mathrm{MWh}$ \\
\hline Overall system annual cost $(J)$ & $287(\mathrm{~m} \$ /$ year)
\end{tabular}

Table 4.6: Clusters of scenario aggregation procedure

\begin{tabular}{|c|r|r|r|r|r|r|}
\hline Cluster \# & $S_{w}(\mathrm{MW})$ & $S_{S}(\mathrm{MW})$ & $P^{*}(\mathrm{MW})$ & $E^{*}(\mathrm{MWh})$ & $J(\mathrm{~m} \$ / \mathrm{yr})$ & Probability \\
\hline 1 & 497.9 & 17.5 & 4.7 & 39.9 & 276.3 & 0.161 \\
\hline 2 & 469.1 & 7.8 & 0.2 & 1.9 & 271.9 & 0.142 \\
\hline 3 & 446.7 & 8.1 & 0 & 0 & 267.7 & 0.132 \\
\hline 4 & 466.0 & 32.8 & 4.1 & 37.1 & 280.6 & 0.128 \\
\hline 5 & 483.6 & 34.6 & 5.4 & 46.2 & 285.6 & 0.122 \\
\hline 6 & 502.4 & 89.8 & 9.0 & 75.2 & 290.9 & 0.1 \\
\hline 7 & 473.6 & 2.4 & 1.2 & 11.1 & 262.2 & 0.096 \\
\hline 8 & 350.7 & 0.0 & 0 & 0 & 252.8 & 0.012 \\
\hline 9 & 515.7 & 96.8 & 50.5 & 442.0 & 298.4 & 0.074 \\
\hline 10 & 613.6 & 167.3 & 194.9 & 1678.0 & 312.4 & 0.033 \\
\hline
\end{tabular}


From the comparison between the two methods, it can be noticed that the results are fairly close to each other. Sensitivity analysis has been conducted to evaluate the effects of the magnitude of uncertainty on the results of two procedures. The sensitivity analysis is done by increasing the variance of random distributions with $10 \%, 20 \%$, $30 \%, 40 \%$, and $50 \%$.

The results of sensitivity analysis using two stage stochastic programming with 5000 samples and scenario aggregation with 1000 samples are given in Table 4.7 and Table 4.8 respectively.

Table 4.7: Sensitivity analysis results for stochastic programming

\begin{tabular}{|r|r|r|r|r|l|}
\hline \multicolumn{1}{|l|}{ Var $+\%$} & \multicolumn{1}{|l|}{$S_{w}(\mathrm{MW})$} & \multicolumn{1}{|l|}{$S_{s}(\mathrm{MW})$} & \multicolumn{1}{|l|}{$P^{*}(\mathrm{MW})$} & $E^{*}(\mathrm{MWh})$ & $\mathrm{J}(\mathrm{m} \$ \mathrm{yr})$ \\
\hline $10 \%$ & 467.2 & 35.6 & 18.9 & 125.9 & 298.5 \\
\hline $20 \%$ & 459.8 & 40.9 & 25.4 & 250.8 & 306.8 \\
\hline $30 \%$ & 438.5 & 44.5 & 56.8 & 342.8 & 315.5 \\
\hline $40 \%$ & 430.5 & 49.8 & 75.8 & 420.8 & 325.8 \\
\hline $50 \%$ & 440.8 & 55.3 & 86.9 & 708.9 & 335.5 \\
\hline
\end{tabular}


Table 4.8: Sensitivity analysis results for scenario aggregation procedure

\begin{tabular}{|l|r|r|r|r|r|}
\hline Var $+\%$ & \multicolumn{1}{|l|}{$S_{w}(\mathrm{MW})$} & \multicolumn{1}{l|}{$S_{s}(\mathrm{MW})$} & \multicolumn{1}{l|}{$P^{*}(\mathrm{MW})$} & \multicolumn{1}{l|}{$(\mathrm{m} \$ / \mathrm{yr})$} \\
\hline $10 \%$ & 471.5 & 36.8 & 20.2 & 176.2 & 291.4 \\
\hline $20 \%$ & 468.4 & 41.8 & 43.3 & 360 & 294.9 \\
\hline $30 \%$ & 445.1 & 44.2 & 55.6 & 469.96 & 297.1 \\
\hline $40 \%$ & 430.19 & 62.8 & 63.7 & 523.8 & 297.6 \\
\hline $50 \%$ & 447.4 & 57.4 & 102.03 & 848.39 & 300.6 \\
\hline
\end{tabular}

It can be seen from sensitivity analysis that the difference between objective values obtained by the two proposed methods increases as the variance increase. Therefore, it can be concluded that the two producers give close results when the uncertainty is low but tend to diverge with increased uncertainty. The other point noticed from the sensitivity analysis is the storage capacities have increased sharply with the increase in variance. This is reasonable since more storage is needed to smooth the fluctuations which are increased with the increased variance.

\subsubsection{Case study two: considering system reliability}

In this case the reliability of the system components is included. The system is supposed to meet the load and the EENS constraint as well. EENS is calculated as

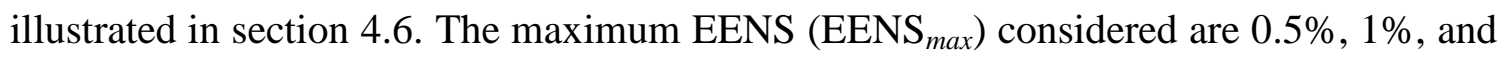
$1.5 \%$. The number of scenarios for this case is chosen to be 5000. The results for this case are shown in Table 4.9. 
Table 4.9: Results for case study two

\begin{tabular}{|c|c|c|c|c|c|}
\hline $\mathrm{EENS}_{\max }$ & $S_{w}(\mathrm{MW})$ & $S_{s}(\mathrm{MW})$ & $P^{*}(\mathrm{MW})$ & $E^{*}(\mathrm{MWh})$ & $J(\mathrm{~m} \$ / \mathrm{yr})$ \\
\hline $0.5 \%$ & 543.1 & 47.2 & 18.7 & 145.2 & 310.8 \\
\hline $1 \%$ & 540.8 & 43.8 & 16.2 & 138.9 & 307.4 \\
\hline $1.5 \%$ & 535.2 & 40.5 & 17.8 & 142.4 & 302.1 \\
\hline
\end{tabular}

From the case study, the total annual cost for the system in case 2 is higher than case 1 which is reasonable, since in case 2 the system has to meet the load and EENS constraint.

In the sensitivity analysis of this case, the energy market reliability is changed. The energy market is assumed to be more reliable by decreasing its failure rate and repair time. The failure rate is changed from $5 / \mathrm{yr}$ to $1 / \mathrm{yr}$, and the repair time from 8 hours to 2 hours. The reliability of the transmission line, connecting energy market to the distribution system, is also increased. The results are given in Table 4.10. 
Table 4.10: Results for case study two with highly reliable energy market

\begin{tabular}{|c|c|c|c|c|c|}
\hline EENS $_{\max }$ & $S_{w}(\mathrm{MW})$ & $S_{s}(\mathrm{MW})$ & $P^{*}(\mathrm{MW})$ & $E^{*}(\mathrm{MWh})$ & $J(\mathrm{~m} \$ / \mathrm{yr})$ \\
\hline $0.5 \%$ & 538.1 & 40.1 & 14.1 & 122.8 & 293.4 \\
\hline $1 \%$ & 536.8 & 38.8 & 14.2 & 119.9 & 292.7 \\
\hline $1.5 \%$ & 525.2 & 36.5 & 13.8 & 118.4 & 290.8 \\
\hline
\end{tabular}

From the sensitivity analysis, the system with high reliability energy market has lower objective value compared with the previous results. The wind and solar farms size has slightly decreased as well, since the market is now more capable to meet system demand. 


\subsection{Summary}

A general hybrid energy system planning model that considers cost of renewables, energy storage, energy market price, system uncertainties, and system reliability has been proposed. An important contribution of this work is a hybrid energy system sizing model that maximizes the benefit from using renewable sources which are highly correlated with time by considering time correlated random distributions of system uncertainties. Thus the hybrid system planning will be more robust and able to take maximum advantage of available renewable resources.

Wind speed, solar irradiance, energy market price, and load data are used to build the stochastic distributions which model system uncertainties. Two stage stochastic programming is used to solve the system uncertainties and to get values of design parameters which will meet load requirements, practical problem constraints, EENS, and reduce overall annual system cost. An alternative method, scenario aggregation procedure has also been developed. This alternative method is simpler and gives close results to the stochastic optimization when the uncertainty is not too high. A case study has been conducted using real data of north Texas distribution system to illustrate the usefulness of the developed methods. 


\section{CHAPTER V}

\section{CONCLUSIONS AND REMARKS}

With the recent developments of energy storage and renewable power generation technologies, large penetration of renewable energy sources is expected. Furthermore, the need of a more reliable and efficient smart grid, many technical challenges need to be solved. The research reported in this dissertation focuses on the development of tools for planning of hybrid energy systems which use renewables connected to energy storage to make the electric grid more reliable and economically efficient. Several important topics in this research are investigated.

The planning of energy storage system for a wind farm, to smooth its output and dispatch power on hourly basis, needs a careful analysis. The optimal size of BESS can be found to be varying due the varying nature of wind power. Therefore a method is needed to determine the proper size that is applicable over the planning horizon. In chapter II, two approaches are proposed using different philosophies. One approach suggested is to meet the needs of dispatched power with a certain level of probability or equivalently to be able to do so for a certain percentage of days of the year. This approach is implemented by finding the frequency distribution of the optimal BESS size for all days of a year or multiple years if so desired. A probability distribution function pdf is then created to represent the frequency distribution. The validity of this pdf is checked by chi-square test. It should be pointed out, if needed; the optimal size can be based on smaller intervals than a day. The other approach suggested is to develop a cost benefit relationship that measures the cost of complete system with varying BESS size 
and find the optimal size which minimizes the overall cost. The SOC and DC-link voltage are important constraints in order to guarantee a proper operation for converters and BESS ability to dispatch the required power respectively.

The planning of hybrid energy systems is discussed in chapters III and IV. In chapter III, a general hybrid energy system planning model that considers cost of renewables, energy storage and energy market price is developed. An important contribution of this suggested procedure is a hybrid energy system sizing model that maximizes the benefit from using renewable sources which are highly correlated with time by considering real time energy market price which changes with time as well. Thus the hybrid system planning will be more robust and able to take maximum advantage of available renewable resources.

Wind speed, solar irradiance, energy market price, and load data are used to optimize hybrid energy system planning using linear programming. The linear programming is used to get values of design parameters which will meet load requirements, practical problem constraints, and reduce overall annual system cost. A case study has been conducted using real data of north Texas distribution system to illustrate the feasibility of the suggested system. Sensitivity analysis which considers changes in load demand and hybrid system configuration has been performed. From the sensitivity analysis, a strong relationship between wind farm and energy storage is indicated.

In chapter IV, the general hybrid energy system of chapter III with system uncertainties, and system reliability is considered. Hybrid energy system sizing model 
that maximizes the benefit from using renewable sources which are highly correlated with time by considering time correlated random distributions of system uncertainties. Thus the hybrid system planning will be more robust and able to take maximum advantage of available renewable resources. Wind speed, solar irradiance, energy market price, and load data are used to build the stochastic distributions which model system uncertainties. A method based on two stage stochastic programming and sample average approximation has been developed to include the system uncertainties and to get values of design parameters which will meet load requirements, practical problem constraints, EENS, and reduce overall annual system cost. An alternative simpler method based on scenario aggregation has also been developed. A case study has been conducted using real data of north Texas distribution system to illustrate the feasibility of the suggested system. The results show that the proposed scenario aggregation procedure gives close results to two stage stochastic programming in the case of system uncertainness are small.

Costs for wind and PV farms have declined over the recent years and this phenomenon is expected to continue, because of the advancement in the technology used in generating electrical power from renewable resources. The research in this dissertation shows that it is practical to use renewable energy system from economical point of view even at the current pricing. Therefore, in the next few years, as the prices fall following the trend, the energy generated from renewable resources will be even more competitive with current traditional technologies to generate energy. 


\section{REFERENCES}

[1] E. A. Committee, "Bottling electricity: storage as a strategic tool for managing variability and capacity concerns in the modern grid," A report by the Electricity Advisory Committee, 2008.

[2] K. Yoshimoto, T. Nanahara, and G. Koshimizu, "New control method for regulating state-of-charge of a battery in hybrid wind power/battery energy storage system," in Power Systems Conference and Exposition, 2006. PSCE'06. 2006 IEEE PES, 2006, pp. 1244-1251.

[3] S. Teleke, M. E. Baran, S. Bhattacharya, and A. Q. Huang, "Optimal control of battery energy storage for wind farm dispatching," IEEE Transactions on Energy Conversion, vol. 25, pp. 787-794, 2010.

[4] PNM Prosperity Energy Storage Project. Available: https://share.pnmresources.com/Public/Pages/Project-Details--Specifics.aspx

[5] G. H. Abbas A. Akhil, Aileen B. Currier, Benjamin C. Kaun, Dan M. Rastler, Stella Bingqing Chen, Andrew L. Cotter, Dale T. Bradshaw, and William D. Gauntlett, "DOE/EPRI 2013 Electricity Storage Handbook in Collaboration with NRECA," Sandia National Laboratories2013.

[6] D. Rastler, Electricity energy storage technology options: a white paper primer on applications, costs and benefits: Electric Power Research Institute, 2010.

[7] (2016). HYBRID WIND AND SOLAR ELECTRIC SYSTEMS. Available: http://energy.gov/energysaver/hybrid-wind-and-solar-electric-systems

[8] A. K. Bansal, R. Kumar, and R. Gupta, "Economic analysis and power management of a small autonomous hybrid power system (SAHPS) using biogeography based optimization (BBO) algorithm," IEEE Transactions on Smart Grid vol. 4, pp. 638-648, 2013.

[9] A. Arabali, M. Ghofrani, M. Etezadi-Amoli, and M. S. Fadali, "Stochastic performance assessment and sizing for a hybrid power system of solar/wind/energy storage," IEEE Transactions on Sustainable Energy vol. 5, pp. 363-371, 2014.

[10] C. Luo and B.-T. Ooi, "Frequency deviation of thermal power plants due to wind farms," IEEE Transactions on Energy Conversion, vol. 21, pp. 708-716, 2006.

[11] C. Han, A. Q. Huang, M. E. Baran, S. Bhattacharya, W. Litzenberger, L. Anderson, et al., "STATCOM impact study on the integration of a large wind 
farm into a weak loop power system," IEEE Transactions on Energy Conversion, vol. 23, pp. 226-233, 2008.

[12] E. Muljadi, C. Butterfield, R. Yinger, and H. Romanowitz, "Energy storage and reactive power compensator in a large wind farm," in AIAA Aerospace Sciences Meeting and Exhibit, 2004, pp. 114-123.

[13] A. Arulampalam, M. Barnes, N. Jenkins, and J. Ekanayake, "Power quality and stability improvement of a wind farm using STATCOM supported with hybrid battery energy storage," in Generation, Transmission and Distribution, IEE Proceedings-, 2006, pp. 701-710.

[14] J. Zeng, B. Zhang, C. Mao, and Y. Wang, "Use of battery energy storage system to improve the power quality and stability of wind farms," in Power System Technology, 2006. PowerCon 2006. International Conference on, 2006, pp. 1-6.

[15] C. Abbey and G. Joos, "Sizing and power management strategies for battery storage integration into wind-diesel systems," in Industrial Electronics, 2008. IECON 2008. 34th Annual Conference of IEEE, 2008, pp. 3376-3381.

[16] F. Baalbergen, P. Bauer, and J. A. Ferreira, "Energy storage and power management for typical 4Q-load," IEEE Transactions on Industrial Electronics, vol. 56, pp. 1485-1498, 2009.

[17] G. O. Cimuca, C. Saudemont, B. Robyns, and M. M. Radulescu, "Control and performance evaluation of a flywheel energy-storage system associated to a variable-speed wind generator," IEEE Transactions on Industrial Electronics, vol. 53, pp. 1074-1085, 2006.

[18] C. Abbey and G. Joos, "Supercapacitor energy storage for wind energy applications," IEEE Transactions on Industry Applications, vol. 43, pp. 769-776, 2007.

[19] S. Teleke, M. E. Baran, A. Q. Huang, S. Bhattacharya, and L. Anderson, "Control strategies for battery energy storage for wind farm dispatching," IEEE Transactions on Energy Conversion, vol. 24, pp. 725-732, 2009.

[20] B. Parkhideh, J. Zeng, S. Baek, S. Bhattacharya, M. Baran, and A. Q. Huang, "Improved wind farm's power availability by battery energy storage systems: Modeling and control," in Industrial Electronics, 2008. IECON 2008. 34th Annual Conference of IEEE, 2008, pp. 784-789. 
[21] J. P. Barton and D. G. Infield, "Energy storage and its use with intermittent renewable energy," IEEE Transactions on Energy Conversion, vol. 19, pp. 441448, 2004.

[22] P. F. Ribeiro, B. K. Johnson, M. L. Crow, A. Arsoy, and Y. Liu, "Energy storage systems for advanced power applications," Proceedings of the IEEE, vol. 89, pp. 1744-1756, 2001.

[23] T. Hennessy and M. Kuntz, "Flow battery storage application with wind power," in Transmission and Distribution Conference and Exhibition, 2005/2006 IEEE PES, 2006, pp. 937-939.

[24] X. Wang, D. Mahinda Vilathgamuwa, and S. Choi, "Determination of battery storage capacity in energy buffer for wind farm," IEEE Transactions on Energy Conversion, vol. 23, pp. 868-878, 2008.

[25] R. Karki, P. Hu, and R. Billinton, "A simplified wind power generation model for reliability evaluation," IEEE Transactions on Energy Conversion, vol. 21, pp. 533-540, 2006.

[26] W. Li, G. Joós, and C. Abbey, "Attenuation of wind power fluctuations in wind turbine generators using a DC bus capacitor based filtering control scheme," in Industry Applications Conference, 2006. 41 st IAS Annual Meeting. Conference Record of the 2006 IEEE, 2006, pp. 216-221.

[27] F. Blaabjerg and Z. Chen, "Power electronics for modern wind turbines," Synthesis Lectures on Power Electronics, vol. 1, pp. 1-68, 2005.

[28] T.-Y. Lee and N. Chen, "Optimal capacity of the battery energy storage system in a power system," IEEE Transactions on Energy Conversion, vol. 8, pp. 667673, 1993.

[29] M. Ceraolo, "New dynamical models of lead-acid batteries," IEEE Transactions on Power Systems, vol. 15, pp. 1184-1190, 2000.

[30] N. W. Miller, J. J. Sanchez-Gasca, W. W. Price, and R. W. Delmerico, "Dynamic modeling of GE 1.5 and 3.6 MW wind turbine-generators for stability simulations," in Power Engineering Society General Meeting, 2003, IEEE, 2003, pp. 1977-1983.

[31] A. AbuElrub and C. Singh, "Long term energy storage capacity optimization in energy buffer system," in PES General Meeting| Conference \& Exposition, 2014 IEEE, 2014, pp. 1-5. 
[32] (2014) Electric Reliability Council of Texas load data. Available: http://www.ercot.com/gridinfo/load/load_hist/

[33] H. Ibrahim, A. Ilinca, and J. Perron, "Energy storage systems - characteristics and comparisons," Renewable and Sustainable Energy Reviews, vol. 12, pp. 1221-1250, 2008.

[34] Z.-L. Gaing, "Particle swarm optimization to solving the economic dispatch considering the generator constraints," IEEE Transactions on Power Systems, vol. 18, pp. 1187-1195, 2003.

[35] Kyotol Protocol to the United Nations_Framework Convention on Climate change, Dec. 1997.

[36] G. D. Burch, "Hybrid Renewable Energy Systems, Hybrid Power Systems Manager Office of Power Technologies, US Department of Energy US DOE Natural Gas," in Renewable Energy Workshops August, 2001.

[37] P. M. R, "Wind and solar power systems," ed. Boca Raton London New York Washington, D.C.:, CRC Press; 1999.

[38] P. Zhang, Y. Wang, W. Xiao, and W. Li, "Reliability evaluation of gridconnected photovoltaic power systems," IEEE Transactions on Sustainable Energy, vol. 3, pp. 379-389, 2012.

[39] A. Mohamed and O. Mohammed, "Connectivity of DC microgrids involving sustainable energy sources," in Industry Applications Society Annual Meeting (IAS), 2011 IEEE, 2011, pp. 1-8.

[40] A. Bidram and A. Davoudi, "Hierarchical structure of microgrids control system," IEEE Transactions on Smart Grid, vol. 3, pp. 1963-1976, 2012.

[41] L. Xu, X. Ruan, C. Mao, B. Zhang, and Y. Luo, "An improved optimal sizing method for wind-solar-battery hybrid power system," IEEE Transactions on Sustainable Energy, vol. 4, pp. 774-785, 2013.

[42] M. Ismail, M. Moghavvemi, and T. Mahlia, "Techno-economic analysis of an optimized photovoltaic and diesel generator hybrid power system for remote houses in a tropical climate," Energy Conversion and Management, vol. 69, pp. 163-173, 2013.

[43] D. S. Koussa and M. Koussa, "A feasibility and cost benefit prospection of grid connected hybrid power system (wind-photovoltaic)-Case study: An Algerian 
coastal site," Renewable and Sustainable Energy Reviews, vol. 50, pp. 628-642, 2015.

[44] B. Bhandari, K.-T. Lee, C. S. Lee, C.-K. Song, R. K. Maskey, and S.-H. Ahn, "A novel off-grid hybrid power system comprised of solar photovoltaic, wind, and hydro energy sources," Applied Energy, vol. 133, pp. 236-242, 2014.

[45] J. Lopes, C. Moreira, and A. Madureira, "Defining control strategies for microgrids islanded operation," IEEE Transactions on Power Systems, vol. 21, pp. 916-924, 2006.

[46] G. T. Heydt, "The next generation of power distribution systems," IEEE Transactions on Smart Grid, vol. 1, pp. 225-235, 2010.

[47] G. T. Heydt, B. H. Chowdhury, M. L. Crow, D. Haughton, B. D. Kiefer, F. Meng, et al., "Pricing and control in the next generation power distribution system," IEEE Transactions on Smart Grid, vol. 3, pp. 907-914, 2012.

[48] A. Mellit, S. Kalogirou, L. Hontoria, and S. Shaari, "Artificial intelligence techniques for sizing photovoltaic systems: A review," Renewable and Sustainable Energy Reviews, vol. 13, pp. 406-419, 2009.

[49] I. J. Ramirez-Rosado and J. L. Bernal-Agustin, "Genetic algorithms applied to the design of large power distribution systems," IEEE Transactions on Power Systems, vol. 13, pp. 696-703, 1998.

[50] G. B. David Feldman, Robert Margolis, Mark Bolinger, Donald Chung, Ran Fu, Joachim Seel, Carolyn Davidson, and Ryan Wiser, "Photovoltaic System Pricing Trends," Sun Shot, US Department of Energy2015.

[51] S. Diaf, D. Diaf, M. Belhamel, M. Haddadi, and A. Louche, "A methodology for optimal sizing of autonomous hybrid PV/wind system," Energy Policy, vol. 35, pp. 5708-5718, 2007.

[52] J. Schlabbach and K.-H. Rofalski, Power system engineering: planning, design, and operation of power systems and equipment: John Wiley \& Sons, 2008.

[53] (2015). Electric Reliability Council of Texas. Available: http://www.ercot.com/gridinfo/load/load_hist/

[54] M. B. Ryan Wiser, "2014 Wind Technologies Market Report," US Department of Eenergy2014. 
[55] A. S. C. Moné, B. Maples, and M. Hand, "2013 Cost of Wind Energy Review," National Renewable Energy Laboratory2013.

[56] P. Xiong. (2016). MATLAB Toolbox for Optimization under Uncertainty. Available: http://xprog.weebly.com/

[57] E. Lantz, R. Wiser, and M. Hand, "The past and future cost of wind energy," National Renewable Energy Laboratory, Golden, CO, Report No. NREL/TP6A20-53510, 2012.

[58] G. Tina, S. Gagliano, and S. Raiti, "Hybrid solar/wind power system probabilistic modelling for long-term performance assessment," Solar Energy, vol. 80, pp. 578-588, 2006.

[59] E. I. Vrettos and S. A. Papathanassiou, "Operating policy and optimal sizing of a high penetration RES-BESS system for small isolated grids," IEEE Transactions on Energy Conversion, vol. 26, pp. 744-756, 2011.

[60] A. Arabali, M. Ghofrani, M. Etezadi-Amoli, M. S. Fadali, and Y. Baghzouz, "Genetic-algorithm-based optimization approach for energy management," IEEE Transactions on Power Delivery, vol. 28, pp. 162-170, 2013.

[61] M. Jafarian and A. Ranjbar, "Fuzzy modeling techniques and artificial neural networks to estimate annual energy output of a wind turbine," Renewable Energy, vol. 35, pp. 2008-2014, 2010.

[62] D. Indhumathy, C. Seshaiah, and K. Sukkiramathi, "Estimation of Weibull Parameters for Wind speed calculation at Kanyakumari in India," International Journal of Innovative Research in Science, Engineering and Technology, vol. 3, pp. 8340-8345, 2014.

[63] A. J. Conejo, F. J. Nogales, and J. M. Arroyo, "Price-taker bidding strategy under price uncertainty," IEEE Transactions on Power Systems, vol. 17, pp. 1081-1088, 2002.

[64] T. Zhang, W. Sheng, X. Song, X. Meng, and C. Shi, "Probabilistic modelling and simulation of stochastic load for power system studies," in Computer Modelling and Simulation (UKSim), 2013 UKSim 15th International Conference on, 2013, pp. 519-524.

[65] P. Jirutitijaroen and C. Singh, "Reliability constrained multi-area adequacy planning using stochastic programming with sample-average approximations," IEEE Transactions on Power Systems, vol. 23, pp. 504-513, 2008. 
[66] W.-K. Mak, D. P. Morton, and R. K. Wood, "Monte Carlo bounding techniques for determining solution quality in stochastic programs," Operations Research Letters, vol. 24, pp. 47-56, 1999.

[67] D. Arthur and S. Vassilvitskii, "k-means++: The advantages of careful seeding," in Proceedings of the eighteenth annual ACM-SIAM symposium on Discrete algorithms, 2007, pp. 1027-1035.

[68] L. Xie, L. Cheng, and Y. Gu, "Reliability assessment at day-ahead operating stage in power systems with wind generation," in System Sciences (HICSS), 2013 46th Hawaii International Conference on, 2013, pp. 2245-2251.

[69] L. Cheng, J. Lin, Y.-Z. Sun, C. Singh, W.-Z. Gao, and X.-M. Qin, "A model for assessing the power variation of a wind farm considering the outages of wind turbines," IEEE Transactions on Sustainable Energy, vol. 3, pp. 432-444, 2012. 